Supporting Information

\title{
Probing Intramolecular Electron Transfer in Redox Tag Processes
}

\section{Naoya Maeta, Hidehiro Kamiya, Yohei Okada*}

Department of Chemical Engineering, Tokyo University of Agriculture and Technology, 2-24-16 Naka-cho,

Koganei, Tokyo 184-8588, Japan

Tel: (+81)-42-388-7068

Fax: (+81)-42-388-7068

E-mail: yokada@cc.tuat.ac.jp

Table of Contents

1. Additional Schemes and Figures p. 2

2. Cartesian Coordinates $\quad$ p. 12

3. General Remarks $\quad$ p. 38

4. Synthesis and Characterization Data p. 39

5. Copies of ${ }^{1} \mathrm{H}$ and ${ }^{13} \mathrm{C}$ NMR Spectra $\quad$ p. 53 


\section{Additional Schemes and Figures}

Scheme S1. Synthesis of Enol Ethers $(\mathbf{2 0}, \mathbf{2 2})$.
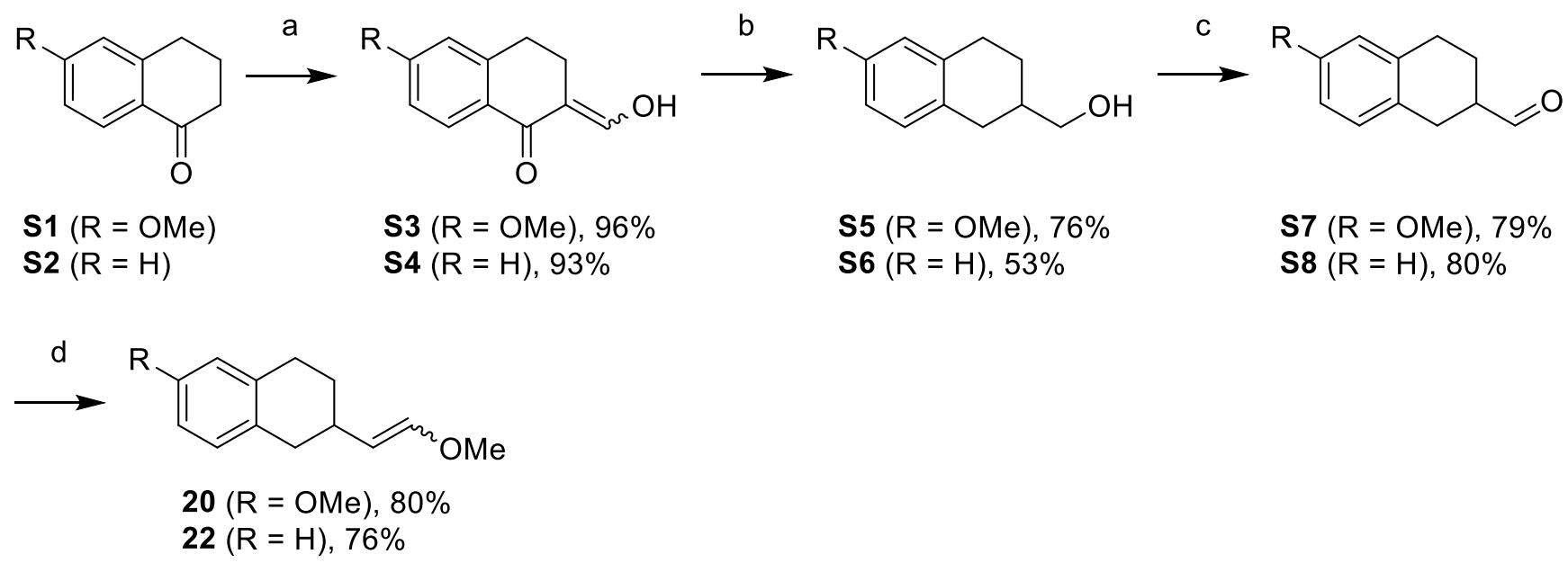

Reagents and conditions: (a) Ethyl formate, $t$-BuOK, toluene, $-78{ }^{\circ} \mathrm{C}-\mathrm{rt}$. (b) Borane $t$-butylamine complex, $\mathrm{BF}_{3} \cdot \mathrm{Et}_{2} \mathrm{O}, \mathrm{CH}_{2} \mathrm{Cl}_{2},-78{ }^{\circ} \mathrm{C}-\mathrm{rt}$. (c) (i) $(\mathrm{COCl})_{2}, \mathrm{DMSO}, \mathrm{CH}_{2} \mathrm{Cl}_{2},-78{ }^{\circ} \mathrm{C}$; (ii) $\mathrm{Et}_{3} \mathrm{~N},-78{ }^{\circ} \mathrm{C}-\mathrm{rt}$. (d) (Methoxymethyl)triphenylphosphonium chloride, $t$-BuOK, THF, $0{ }^{\circ} \mathrm{C}$. 
Figure S1. Spin Density Distributions in the Radical Cations $\left(\mathbf{1 0}^{\bullet+}, \mathbf{1 2}^{\mathbf{*}}\right)$.

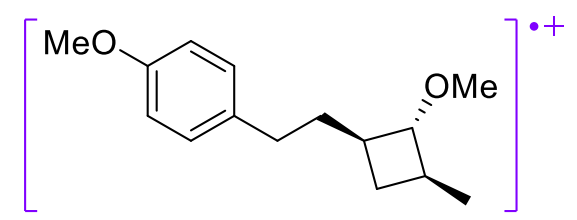

$3^{\circ+}$

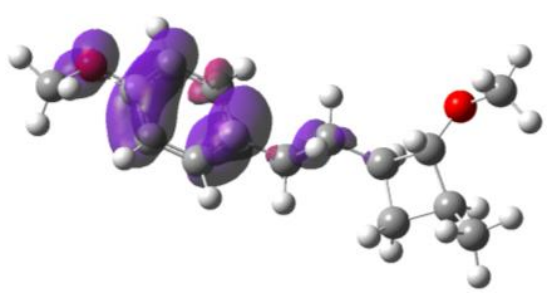

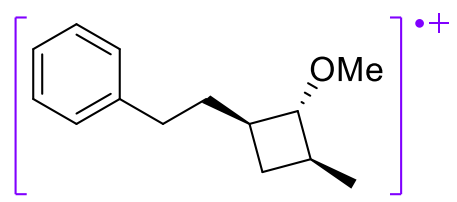

$15^{\circ+}$

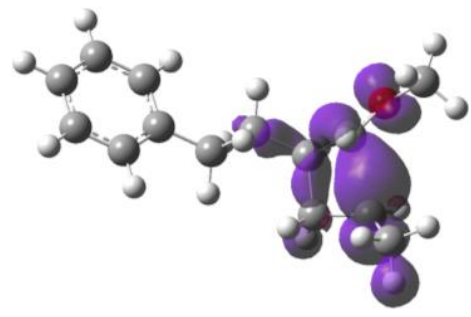


Figure S2. Relative Energies of the Enol Ether (20).
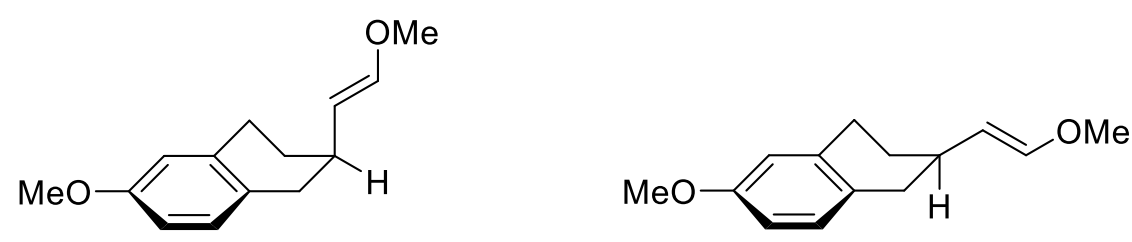

+1.8 kcal $/ \mathrm{mol}$

$+1.4 \mathrm{kcal} / \mathrm{mol}$
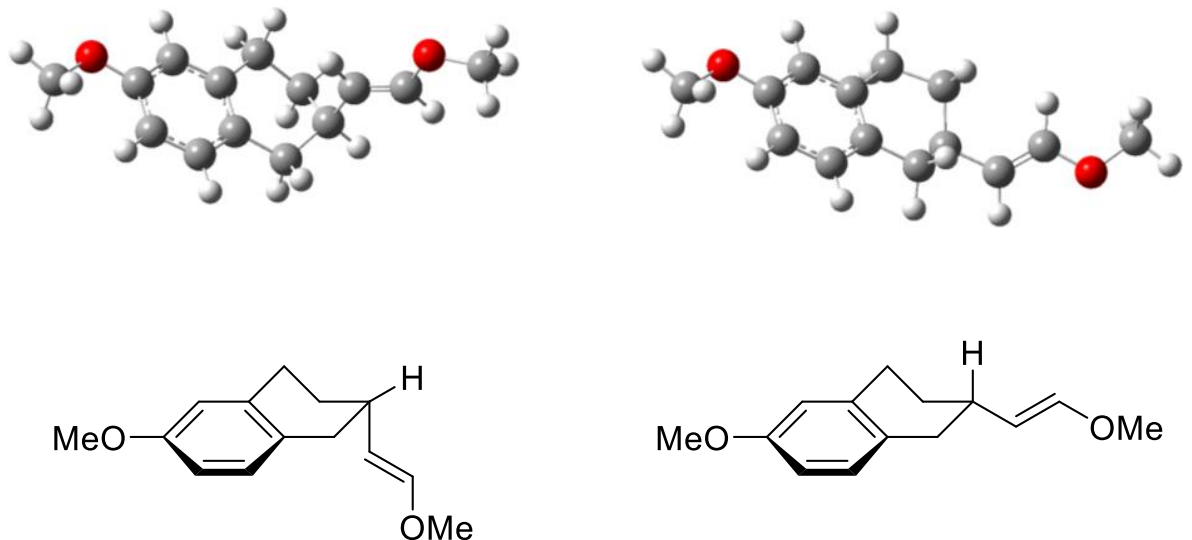

$+1.8 \mathrm{kcal} / \mathrm{mol}$

$0.0 \mathrm{kcal} / \mathrm{mol}$
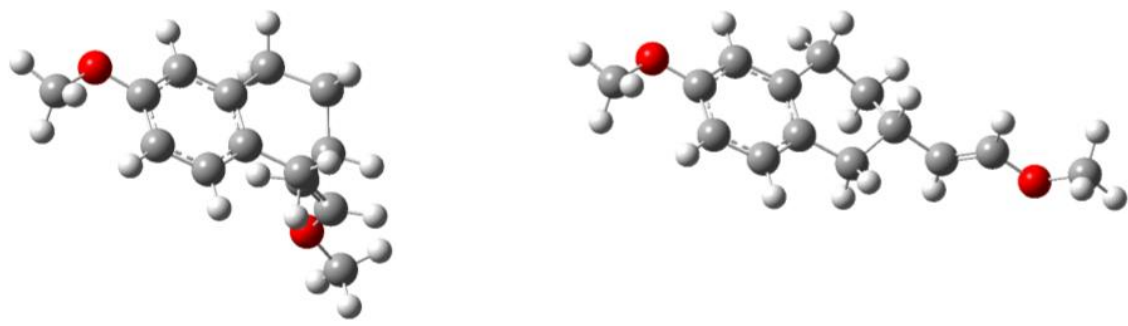
Figure S3. Relative Energies of the Enol Ether Radical Cation $\left(\mathbf{2 0}^{\circ+}\right)$.
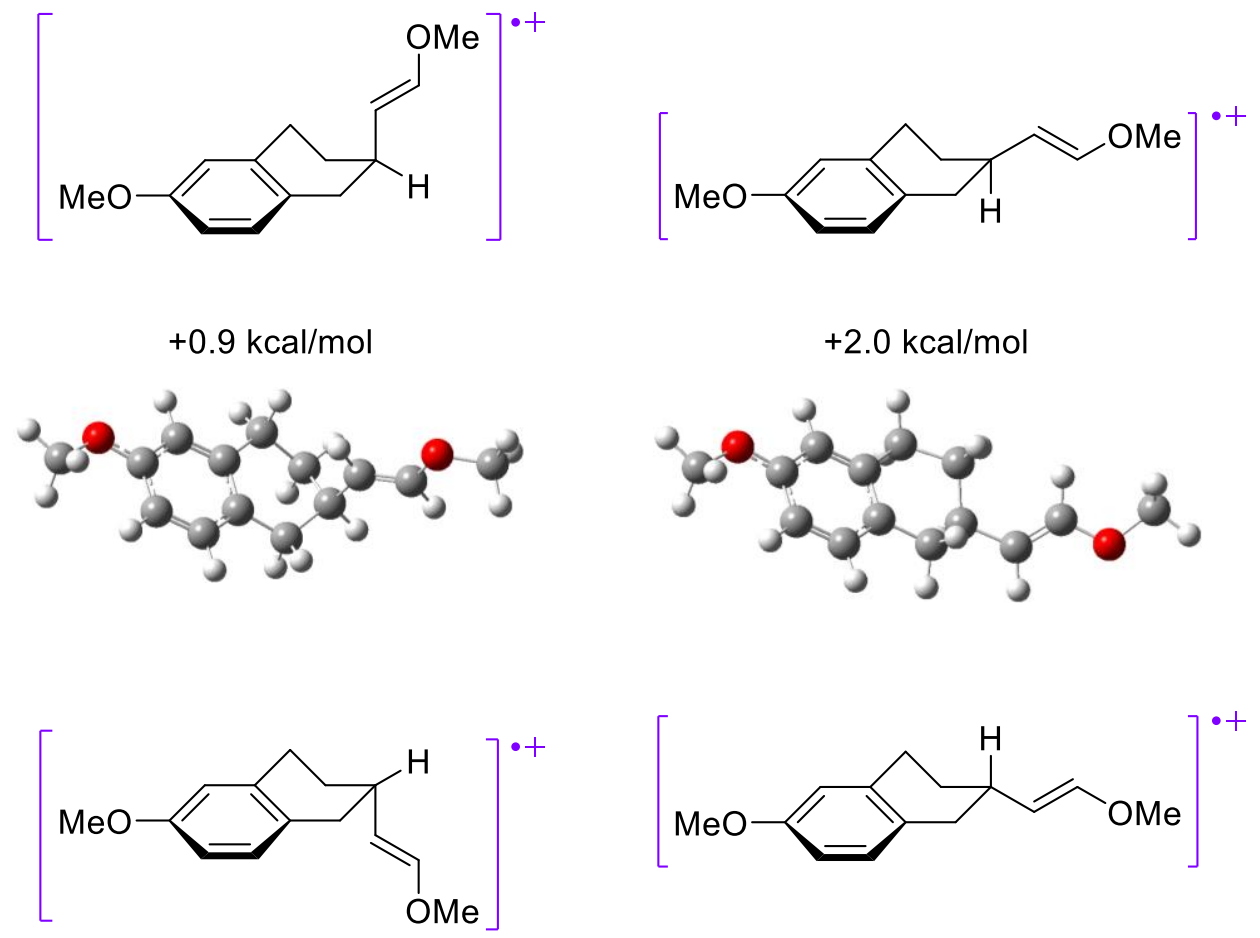
$+0.9 \mathrm{kcal} / \mathrm{mol}$

$0.0 \mathrm{kcal} / \mathrm{mol}$
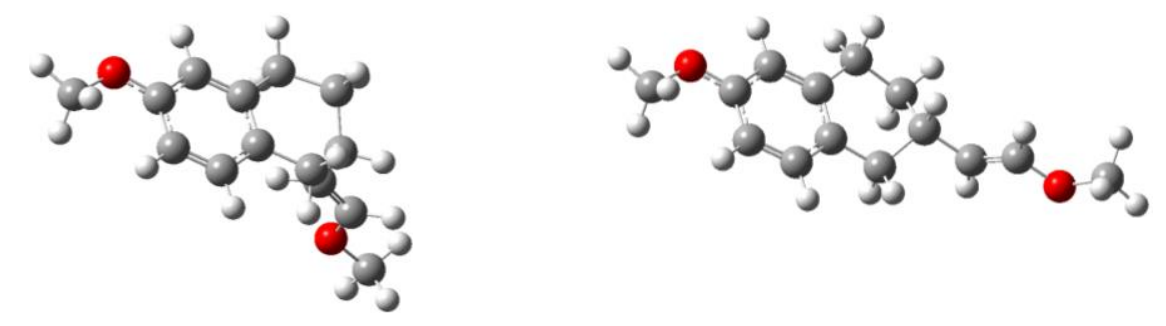
Figure S4. Relative Energies of the Enol Ether (22).

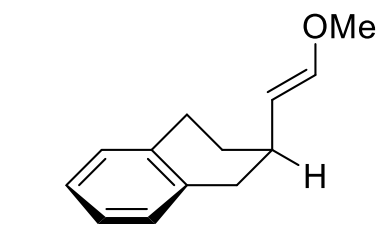

$+1.7 \mathrm{kcal} / \mathrm{mol}$
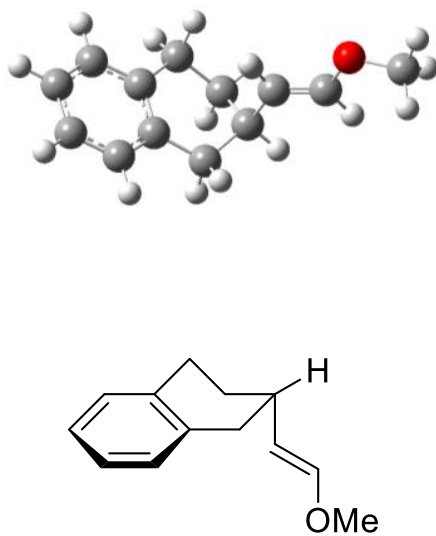

$+1.7 \mathrm{kcal} / \mathrm{mol}$

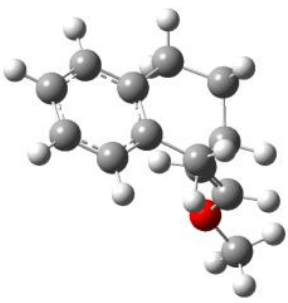

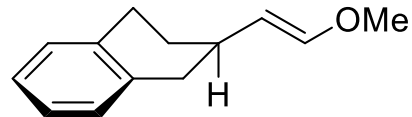

$+1.4 \mathrm{kcal} / \mathrm{mol}$
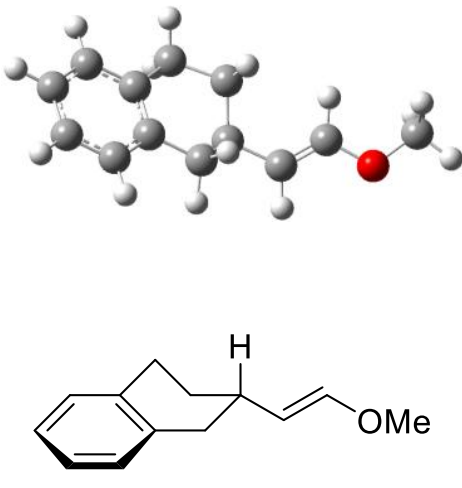

$0.0 \mathrm{kcal} / \mathrm{mol}$

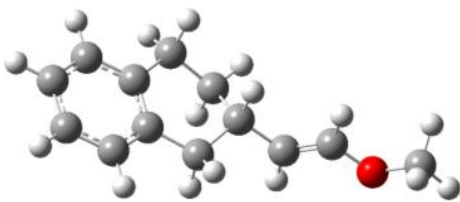


Figure S5. Relative Energies of the Enol Ether Radical Cation $\left(\mathbf{2 2}^{\mathbf{+}}\right)$

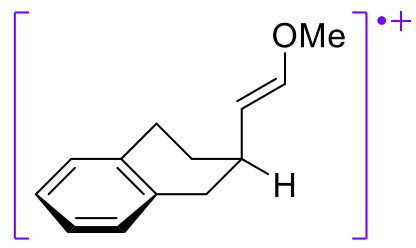

$+1.3 \mathrm{kcal} / \mathrm{mol}$
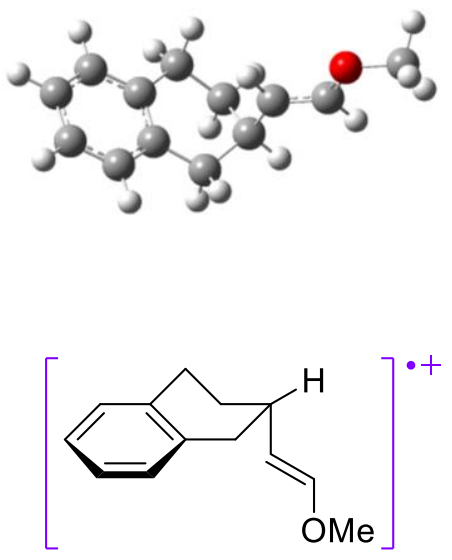

$+1.3 \mathrm{kcal} / \mathrm{mol}$

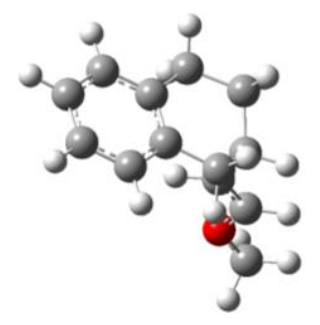

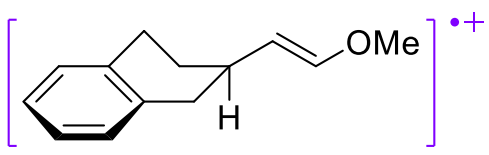

$+1.7 \mathrm{kcal} / \mathrm{mol}$
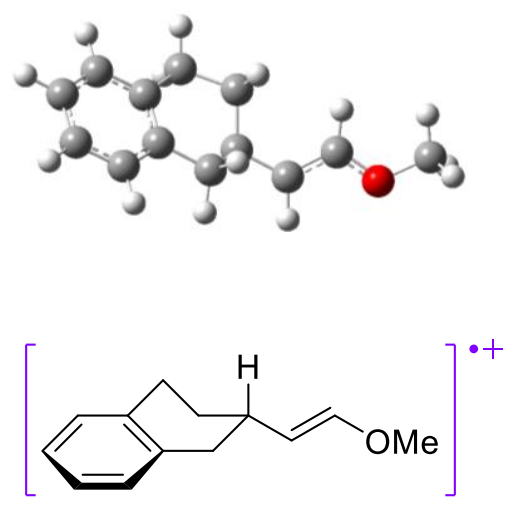

$0.0 \mathrm{kcal} / \mathrm{mol}$

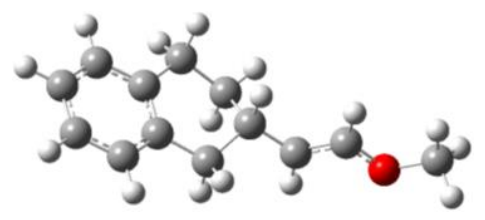


Figure S6. HOMO of the Enol Ethers $(\mathbf{2 0}, \mathbf{2 2})$ and Spin Density Distributions in the Enol Ether Radical Cations $\left(\mathbf{2 0}^{\circ+}, \mathbf{2 2}^{\circ+}\right)$.<smiles>COC=C[C@@H]1CCc2cc(OC)ccc2C1</smiles>

20

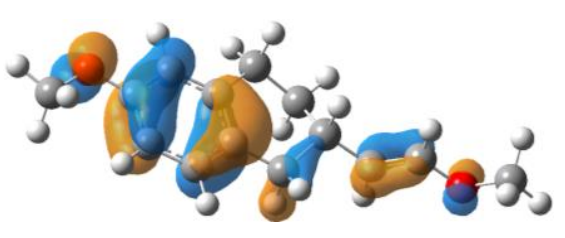<smiles>COC=C[C@@H]1CCc2cc(OC)ccc2C1</smiles>

$20^{\circ+}$

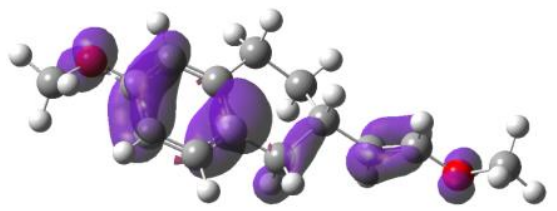

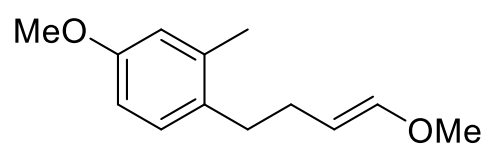

22
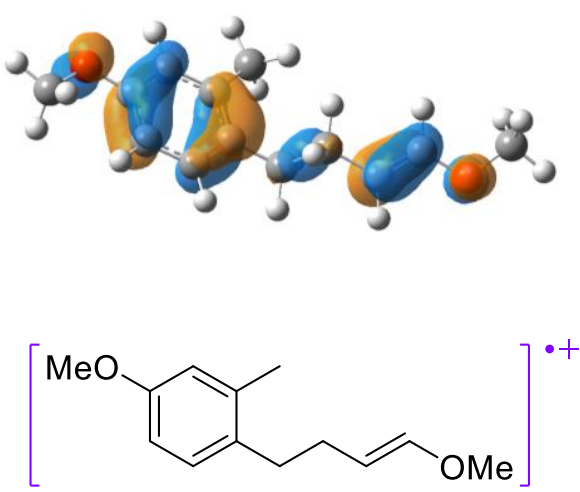

$22^{\cdot+}$

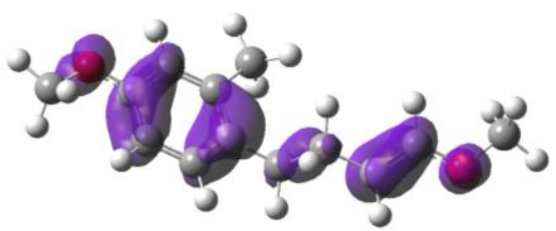


Figure S7. HOMO of the Enol Ethers $(\mathbf{2 1}, \mathbf{2 3})$ and Spin Density Distributions in the Enol Ether Radical Cations $\left(21^{\cdot+}, \mathbf{2 3}^{\circ+}\right)$.<smiles>COC=C[C@H]1CCc2ccccc2C1</smiles>

21
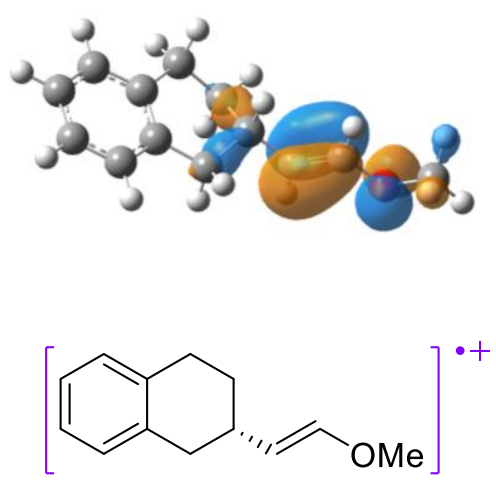

$21^{\circ+}$

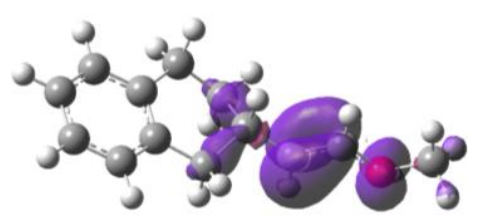

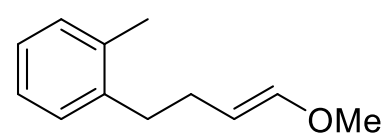

23
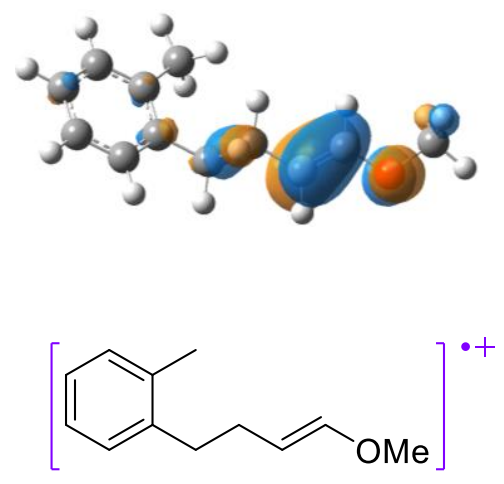

$23^{\circ+}$

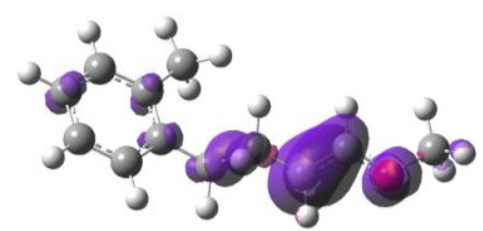




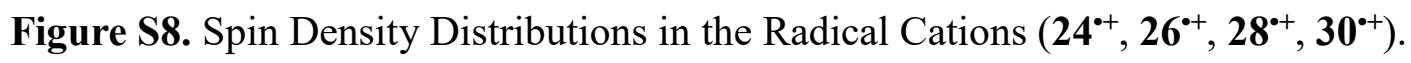

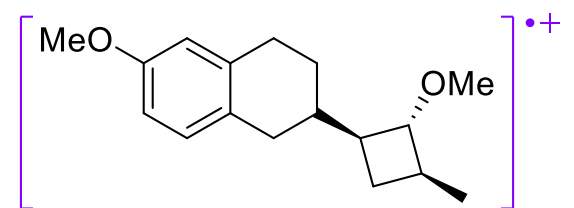

$24^{\circ+}$
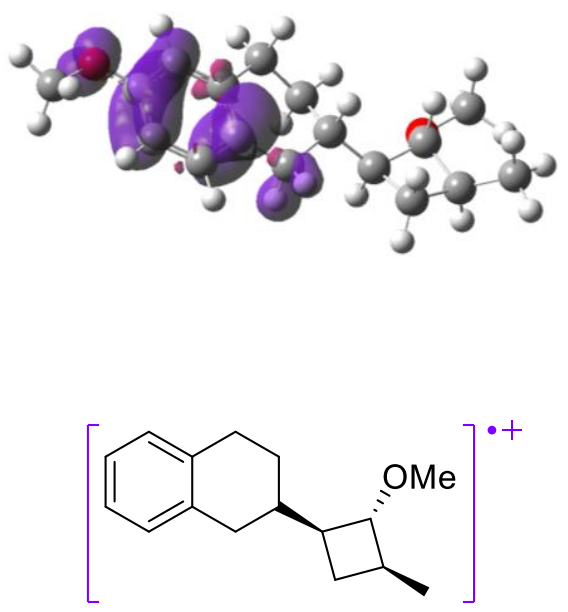

$26^{\circ+}$

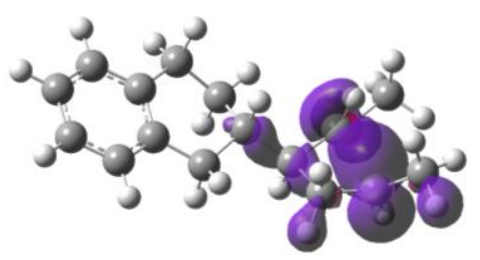

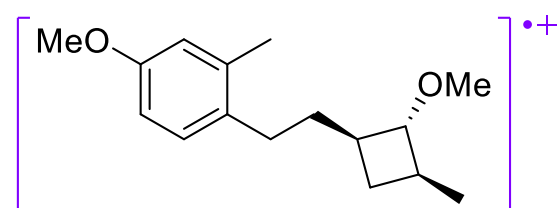

$28^{\cdot+}$
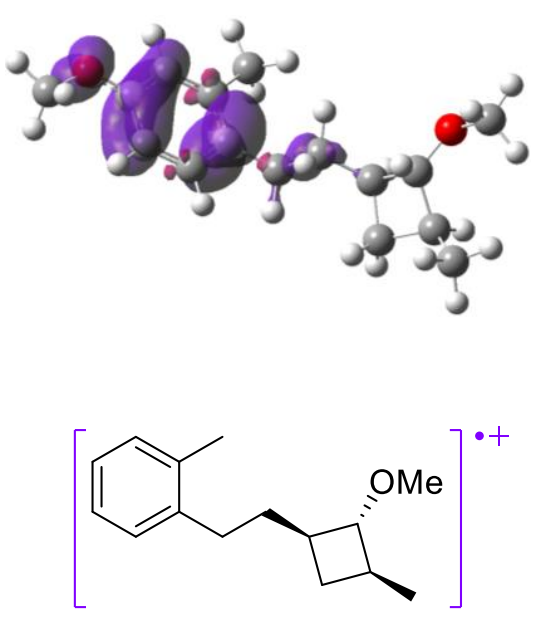

$30^{\circ+}$

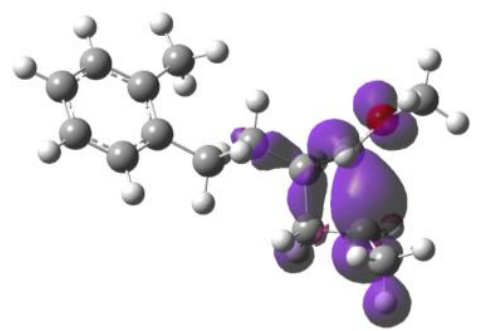


2. Cartesian Coordinates.

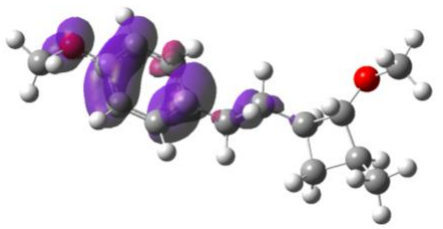

$3^{\cdot+}$

Total Energy $=-734.933282$

Atom $\mathrm{X}$ Y

$\mathrm{Z}$

$\begin{array}{llll}\text { C } & 3.36431617 & 0.34582904 & 0.31023433\end{array}$

$\begin{array}{llll}\text { C } & 2.23965491 & -0.06065815 & -0.65991034\end{array}$

$\mathrm{H} \quad 2.87959916 \quad-2.00460857 \quad-1.64246446$

$\begin{array}{llll}\mathrm{H} & 2.37592343 & -2.13995266 & 0.05238784\end{array}$

$\mathrm{H} \quad 4.81109573 \quad-0.59983875 \quad-0.98923331$

$\begin{array}{llll}\mathrm{H} & 3.05001007 & 0.23450986 & 1.35588785\end{array}$

$\begin{array}{llll}\mathrm{H} & 2.41771668 & 0.43393463 & -1.61677712\end{array}$

$\begin{array}{llll}\text { C } & -1.61169252 & -0.16023559 & -0.82478463\end{array}$

$\begin{array}{llll}\text { C } & -2.27419178 & -1.14954112 & -0.04336124\end{array}$

$\begin{array}{llll}\text { C } & -2.29801351 & 1.04735598 & -1.12056994\end{array}$

$\begin{array}{llll}\text { C } & -3.54646569 & -0.96584077 & 0.41690071\end{array}$

$\mathrm{H} \quad-1.75418405 \quad-2.06970523 \quad 0.18113450$

$\begin{array}{llll}\text { C } & -3.56785856 & 1.25667518 & -0.67275747\end{array}$

$\begin{array}{llll}\text { C } & -4.21674681 & 0.25182622 & 0.11054456\end{array}$

$\mathrm{H} \quad-4.03451813 \quad-1.73010180 \quad 0.99931030$

$\mathrm{H} \quad-4.11202872 \quad 2.16379573 \quad-0.88892230$

$\begin{array}{llll}\text { C } & 0.80055633 & 0.15333333 & -0.21673488\end{array}$

$\begin{array}{llll}\mathrm{H} & 0.62352662 & -0.35959202 & 0.73029030\end{array}$

$\mathrm{H} \quad 0.61981033 \quad 1.21650242 \quad-0.04686625$

$\begin{array}{llll}\text { C } & -0.21341902 & -0.37151070 & -1.27854526\end{array}$

$\begin{array}{llll}\mathrm{H} & -0.02959654 & -1.43340739 & -1.44196747\end{array}$

$\mathrm{H} \quad-0.04012468 \quad 0.15820835 \quad-2.21518132$

$\begin{array}{llll}\text { O } & -5.43005598 & 0.54826224 & 0.50158909\end{array}$

C $\quad-6.21034614 \quad-0.36693725 \quad 1.31651094$

$\begin{array}{llll}\mathrm{H} & -7.14741702 & 0.14699226 & 1.48716442\end{array}$

$\begin{array}{llll}\mathrm{H} & -6.37531593 & -1.29209689 & 0.77123486\end{array}$

$\mathrm{H} \quad-5.69614572 \quad-0.54809648 \quad 2.25645680$

$\begin{array}{llll}\text { O } & 3.87091044 & 1.64672906 & 0.09955996\end{array}$

$\begin{array}{llll}\mathrm{C} & 4.83735880 & 2.05022443 & 1.06400055\end{array}$

$\begin{array}{llll}\mathrm{H} & 4.43063232 & 1.98531024 & 2.07782839\end{array}$ 


$\begin{array}{cccc}\mathrm{H} & 5.74205349 & 1.44019739 & 1.00542615 \\ \mathrm{H} & 5.09348959 & 3.08445644 & 0.84712379 \\ \mathrm{C} & 4.97961191 & -1.69801352 & 0.86598968 \\ \mathrm{H} & 4.36552746 & -1.95399002 & 1.73158709 \\ \mathrm{H} & 5.33921858 & -2.63127085 & 0.42793329 \\ \mathrm{H} & 5.84930935 & -1.14449130 & 1.22316596 \\ \mathrm{H} & -1.80322911 & 1.80281742 & -1.71361405\end{array}$




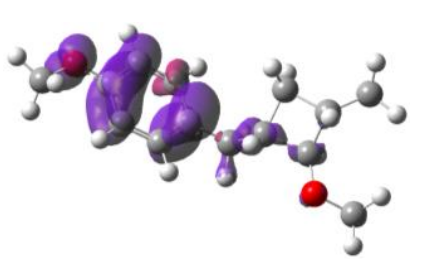

$10^{\circ+}$

Total Energy $=-695.635563$

\begin{tabular}{|c|c|c|c|}
\hline Atom & $\mathrm{X}$ & Y & Z \\
\hline $\mathrm{C}$ & -1.69979626 & 1.36627601 & -0.54015261 \\
\hline C & -3.17566152 & 0.96361373 & -0.81177787 \\
\hline $\mathrm{C}$ & -2.94454485 & -0.26346638 & 0.12407088 \\
\hline $\mathrm{C}$ & -1.43200536 & -0.09217754 & -0.09200102 \\
\hline H & -1.11894295 & 1.74514314 & -1.37714210 \\
\hline $\mathrm{H}$ & -1.62819014 & 2.06782396 & 0.29149700 \\
\hline $\mathrm{H}$ & -3.27115083 & 0.59695044 & -1.83503539 \\
\hline $\mathrm{H}$ & -3.23320548 & -0.01695073 & 9394 \\
\hline $\mathrm{H}$ & -1.13549460 & -0.69711126 & -0.94914124 \\
\hline $\mathrm{C}$ & -0.50511041 & -0.39170821 & 1.11345754 \\
\hline $\mathrm{H}$ & -0.78776976 & 0.24592244 & 1.94946526 \\
\hline $\mathrm{H}$ & -0.66841140 & -1.43277891 & 1.40096587 \\
\hline $\mathrm{O}$ & -3.44015120 & -1.53212322 & -0.22703803 \\
\hline$\tau$ & -4.79965822 & -1.74816698 & 0.14328015 \\
\hline $\mathrm{H}$ & -4.94224305 & -1.59020402 & 1.21609262 \\
\hline$H$ & -5.47316900 & -1.08713703 & -0.4068 \\
\hline $\mathrm{H}$ & -5.03605447 & -2.78031258 & 4772 \\
\hline $\mathrm{C}$ & -4.27791223 & 1.95770991 & -0.48702343 \\
\hline $\mathrm{H}$ & -4.21212387 & 2.28919595 & 0.55080121 \\
\hline $\mathrm{H}$ & -4.20100276 & 2.84135700 & 44271 \\
\hline $\mathrm{H}$ & -5.26705937 & 1.52427990 & -0.64070889 \\
\hline $\mathrm{C}$ & 0.92462343 & -0.19028999 & 0.76438094 \\
\hline $\mathrm{C}$ & 1.60752675 & 1.00362629 & 1.11168123 \\
\hline $\mathrm{C}$ & 1.62893026 & -1.19313494 & 0.03615148 \\
\hline $\mathrm{C}$ & 2.91438566 & 1.18596561 & 0.76702674 \\
\hline $\mathrm{H}$ & 1.08496585 & 1.77068783 & 1.66416024 \\
\hline $\mathrm{C}$ & 2.93842523 & -1.03754974 & -0.31711670 \\
\hline $\mathrm{H}$ & 1.11133861 & -2.10353051 & -0.23048492 \\
\hline $\mathrm{C}$ & 3.60613373 & 0.16526834 & 0.04449269 \\
\hline $\mathrm{H}$ & 3.45698579 & 2.08271908 & 1.02614619 \\
\hline $\mathrm{H}$ & 3.45635421 & -1.81364230 & -0.85656555 \\
\hline $\mathrm{O}$ & 4.85681822 & 0.43338192 & -0.23657765 \\
\hline$r$ & 5.68834792 & -0.51033860 & -0.96235233 \\
\hline
\end{tabular}


$\mathrm{H} \quad 6.64786407 \quad-0.01827572 \quad-1.05522163$

$\mathrm{H} \quad 5.78327163 \quad-1.42836929 \quad-0.38888042$

$\begin{array}{llll}\mathrm{H} & 5.25859629 & -0.69960195 & -1.94194859\end{array}$ 


\begin{tabular}{|c|c|c|c|}
\hline \multirow[b]{2}{*}{ Atom } & $12^{++}$ & \multicolumn{2}{|c|}{ Total Energy $=-581.092016$} \\
\hline & $\mathrm{X}$ & $\mathrm{Y}$ & $\mathrm{Z}$ \\
\hline $\mathrm{C}$ & -0.80412204 & 1.31714850 & -0.43548217 \\
\hline $\mathrm{C}$ & -2.26721584 & 1.37435139 & -0.75571036 \\
\hline $\mathrm{C}$ & -2.11639405 & -0.44823274 & 0.27598312 \\
\hline $\mathrm{C}$ & -0.69569847 & -0.20237059 & -0.14488985 \\
\hline $\mathrm{H}$ & -0.16923584 & 1.63287661 & -1.26303233 \\
\hline $\mathrm{H}$ & -0.56705916 & 1.91185195 & 0.44451286 \\
\hline $\mathrm{H}$ & -2.56161782 & 0.93909286 & -1.70501698 \\
\hline $\mathrm{H}$ & -2.48235393 & -0.04811402 & 1.21965114 \\
\hline $\mathrm{H}$ & -0.52751420 & -0.74135108 & -1.07614562 \\
\hline $\mathrm{C}$ & 0.34658909 & -0.63558815 & 0.91345253 \\
\hline $\mathrm{H}$ & 0.14672473 & -0.11399181 & 1.84919489 \\
\hline $\mathrm{H}$ & 0.20900307 & -1.70191512 & 1.09931742 \\
\hline $\mathrm{O}$ & -2.75785393 & -1.45451459 & -0.23074611 \\
\hline $\mathrm{C}$ & -4.09341990 & -1.76369713 & 0.26369008 \\
\hline $\mathrm{H}$ & -4.17561643 & -1.48007282 & 1.30891316 \\
\hline $\mathrm{H}$ & -4.80957782 & -1.22419028 & -0.34923037 \\
\hline $\mathrm{H}$ & -4.21210782 & -2.83312859 & 0.13832698 \\
\hline $\mathrm{C}$ & -3.20493768 & 2.33994977 & -0.14430494 \\
\hline $\mathrm{H}$ & -2.96761715 & 2.55518381 & 0.89633399 \\
\hline $\mathrm{H}$ & -3.10562546 & 3.28486669 & -0.69849588 \\
\hline $\mathrm{H}$ & -4.24234980 & 2.02531903 & -0.23985744 \\
\hline $\mathrm{C}$ & 1.76239290 & -0.36768846 & 0.46091641 \\
\hline $\mathrm{C}$ & 2.47322204 & 0.73665936 & 0.93714554 \\
\hline $\mathrm{C}$ & 2.38667431 & -1.22101728 & -0.45475655 \\
\hline $\mathrm{C}$ & 3.77488431 & 0.98442154 & 0.50917609 \\
\hline $\mathrm{H}$ & 2.01134867 & 1.40261504 & 1.65439840 \\
\hline $\mathrm{C}$ & 3.68632260 & -0.97621486 & -0.88395423 \\
\hline $\mathrm{H}$ & 1.85548667 & -2.08798670 & -0.82670288 \\
\hline $\mathrm{C}$ & 4.38459041 & 0.12968815 & -0.40393934 \\
\hline $\mathrm{H}$ & 4.31164980 & 1.84155500 & 0.89224813 \\
\hline $\mathrm{H}$ & 4.15566479 & -1.65028933 & -1.58742782 \\
\hline $\mathrm{H}$ & 5.39630304 & 0.31933854 & -0.73497669 \\
\hline
\end{tabular}


$15^{++}$

Total Energy $=-620.390487$

$\begin{array}{llcc}\text { Atom } & \text { X } & \text { Y } & \text { Z } \\ \text { C } & -1.93054285 & -1.47946312 & 0.64077115 \\ \text { C } & -3.38296908 & -1.20590594 & 0.39291478 \\ \text { C } & -2.47701685 & 0.58438477 & -0.25538639 \\ \text { C } & -1.40068529 & -0.01909848 & 0.60015856 \\ \text { H } & -1.74873748 & -1.96582341 & 1.59907066 \\ \text { H } & -1.49701500 & -2.08343770 & -0.15426879 \\ \text { H } & -3.92479648 & -0.74995468 & 1.21475950 \\ \text { H } & -2.53474301 & 0.33738160 & -1.31366408 \\ \text { H } & -1.47655735 & 0.42585761 & 1.59185282 \\ \text { C } & 2.48847335 & -0.19247505 & 0.39731081 \\ \text { C } & 3.08047575 & -1.12373727 & -0.46010780 \\ \text { C } & 3.20418754 & 0.96435676 & 0.71811924 \\ \text { C } & 4.35155860 & -0.90617630 & -0.98432571 \\ \text { H } & 2.54491012 & -2.02963178 & -0.71489940 \\ \text { C } & 4.47544227 & 1.18634659 & 0.19642200 \\ \text { C } & 5.05340383 & 0.25126724 & -0.65821531 \\ \text { H } & 4.79446894 & -1.64149599 & -1.64247544 \\ \text { H } & 5.01516962 & 2.08576697 & 0.46057849 \\ \text { C } & 0.01197557 & 0.18462417 & 0.03063255 \\ \text { H } & 0.07454652 & -0.26323889 & -0.96290629 \\ \text { H } & 0.18763361 & 1.25552254 & -0.08846222 \\ \text { C } & 1.09684809 & -0.41683512 & 0.94256142 \\ \text { H } & 0.92036076 & -1.48701747 & 1.05938333 \\ \text { H } & 1.01359367 & 0.03111410 & 1.93429203 \\ \text { O } & -3.05055582 & 1.67936285 & 0.13291929 \\ \text { C } & -4.02060032 & 2.31725080 & -0.74863226 \\ \text { H } & -3.71650799 & 2.19082175 & -1.78361770 \\ \text { H } & -4.99049904 & 1.86426920 & -0.56463576 \\ & -4.02647548 & 3.36326070 & -0.46794993 \\ \text { H } & -4.18774432 & -1.90081447 & -0.63409539 \\ \text { H } & -3.61849308 & -2.12408028 & -1.53498545 \\ \text { H } & -2.86440334 & -0.20521680 \\ \text { H } & -1.35709950 & -0.88376930\end{array}$


$\begin{array}{llll}\mathrm{H} & 2.76562165 & 1.69444325 & 1.38675597\end{array}$

$\mathrm{H} \quad 6.04222065 \quad 0.42049511 \quad-1.06196185$ 


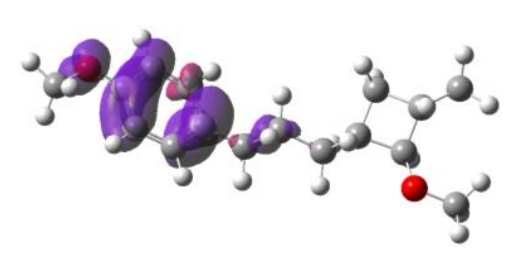

$16^{\circ+}$

Total Energy $=-774.231095$

Atom $\mathrm{X}$ $\mathrm{Y} \quad \mathrm{Z}$

C $\quad-4.45972736$

1.44188166

$-0.57188858$

C $\quad-4.13333278$

1.07429458

$-0.72773539$

C $\quad-2.64407059$

$-0.26088430$

0.00037338

$\mathrm{H} \quad-2.47295784$

$-0.06108086$

$-0.33219928$

$\mathrm{H} \quad-2.78474124$

1.93038336

$-1.41425921$

$\mathrm{H} \quad-4.67198820$

2.03510524

0.32781514

H $\quad-4.31721972$

0.84972485

$-1.77497455$

$-0.16585953$

1.07846586

$\mathrm{H} \quad-2.44269194$

$-0.54799840$

$-1.28917770$

C $\quad-1.60115224$

$-0.47328659$

0.69702039

$\mathrm{H} \quad-1.80428152$

0.03606361

1.64310405

$\mathrm{H} \quad-1.68984332$

$-1.54577761$

0.89158725

O $\quad-4.67822175$

$-1.47776683$

$-0.46983304$

C $\quad-6.02968344$

$-1.69037827$

$-0.07838672$

H $\quad-6.13384399$

$-1.65271325$

1.01047785

H $\quad-6.69856142$

$-0.94942213$

$-0.52328222$

$\mathrm{H} \quad-6.31405234$

$-2.67927602$

$-0.43025432$

C $\quad-5.50698443$

2.01719251

$-0.15862648$

$\mathrm{H} \quad-5.32833197$

2.20375821

0.90219854

$\mathrm{H} \quad-5.48366253$

2.98066534

$-0.67205593$

$\mathrm{H} \quad-6.51499020$

1.61265743

$-0.26287969$

C $\quad-0.17266010$

$-0.15800672$

0.24527296

$\mathrm{H} \quad-0.06836532$

0.90967243

0.04583278

H $\quad 0.04446691$

$-0.67953483$

$-0.68874803$

C $\quad 0.87523973$

$-0.57591930$

1.31879836

$\mathrm{H} \quad 0.76269518$

$-1.64176622$

1.51861453

$\mathrm{H} \quad 0.66922743$

$-0.02873103$

2.23871640

C $\quad 2.25707763$

$-0.28915193$

0.85472020

C 2.86347806

0.97024543

1.10573553

C 2.98220660

$-1.26081275$

0.10690366

C $\quad 4.11677084$

1.24547411

0.64714307

$\mathrm{H} \quad 2.32106760$

1.71277618

1.67288760

C $\quad 4.23924137$

$-1.01124175$

$-0.36374853$

$\mathrm{H}$

2.52254638

$-2.22004042$

$-0.08359112$ 


$\begin{array}{lrrr}\mathrm{C} & 4.82911452 & 0.25735464 & -0.10191190 \\ \mathrm{H} & 4.60131654 & 2.19296935 & 0.82914047 \\ \mathrm{H} & 4.77579266 & -1.76118319 & -0.92161792 \\ \mathrm{C} & 6.85749261 & -0.27598517 & -1.28879972 \\ \mathrm{H} & 7.07069362 & -1.17408185 & -0.71579151 \\ \mathrm{H} & 6.35988158 & -0.51339781 & -2.22514209 \\ \mathrm{H} & 7.76489633 & 0.28525788 & -1.47031641 \\ \mathrm{O} & 6.02081866 & 0.61683891 & -0.50551390\end{array}$




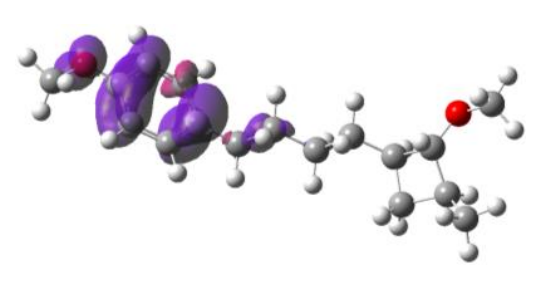

$18^{\circ+}$

Total Energy $=-813.528837$

\begin{tabular}{|c|c|c|c|}
\hline Atom & $\mathrm{X}$ & $\mathrm{Y}$ & $\mathrm{Z}$ \\
\hline $\mathrm{C}$ & 4.00042589 & -1.46791866 & -0.47764204 \\
\hline $\mathrm{C}$ & 5.38653369 & -0.89679294 & -0.06726618 \\
\hline $\mathrm{C}$ & 4.64008453 & 0.39256897 & 0.37827347 \\
\hline $\mathrm{C}$ & 3.43700394 & -0.01929323 & -0.48710839 \\
\hline $\mathrm{H}$ & 3.94857263 & -2.03628007 & -1.40372301 \\
\hline $\mathrm{H}$ & 3.56184125 & -2.062 & 0.32608707 \\
\hline $\mathrm{H}$ & 5.96 & -0.665 & 476 \\
\hline $\mathrm{H}$ & 4.39574479 & 0.34534865 & 1.44763727 \\
\hline $\mathrm{H}$ & 3.57001757 & 0.41460672 & -1.48110239 \\
\hline$C$ & 2.02994885 & 0.2 & 9722 \\
\hline $\mathrm{H}$ & 1.91315245 & -0.18643708 & 1.01495831 \\
\hline $\mathrm{H}$ & 1.90150743 & 1.33835171 & 0.14859579 \\
\hline $\mathrm{C}$ & 0.93534216 & -0.27 & -0.90 \\
\hline $\mathrm{H}$ & 1.05931054 & -1.35322644 & -1.03811650 \\
\hline $\mathrm{H}$ & 1.04875625 & 0.17402552 & -1.89744313 \\
\hline $\mathrm{O}$ & 5.17523751 & 47148 & 0.06997065 \\
\hline $\mathrm{C}$ & 6.23519853 & 2.06526063 & 0.93043350 \\
\hline $\mathrm{H}$ & 5.91604411 & 2.05683973 & 1.97744808 \\
\hline $\mathrm{H}$ & 7.10809164 & 1.41650785 & 0.82223116 \\
\hline $\mathrm{H}$ & 6.51202681 & 3.07893220 & 0.65007846 \\
\hline $\mathrm{C}$ & 6.23194033 & -1.67339400 & 0.92886235 \\
\hline $\mathrm{H}$ & 5.67446861 & -1.86355485 & 1.84823387 \\
\hline $\mathrm{H}$ & 6.52958099 & -2.64012594 & 0.51733959 \\
\hline $\mathrm{H}$ & 7.14260199 & -1.13418570 & 1.19460764 \\
\hline $\mathrm{C}$ & -0.47227044 & 0.01023605 & -0.37967111 \\
\hline $\mathrm{H}$ & -0.61421785 & 1.08557017 & -0.25709794 \\
\hline $\mathrm{H}$ & -0.60006218 & -0.44217512 & 0.60550208 \\
\hline $\mathrm{C}$ & -1.56800971 & -0.54030653 & -1.33951818 \\
\hline $\mathrm{H}$ & -1.43844674 & -0.07848514 & -2.31825720 \\
\hline $\mathrm{H}$ & -1.42731385 & -1.61647785 & -1.44492354 \\
\hline $\mathrm{C}$ & -2.93065681 & -0.25562902 & -0.82028471 \\
\hline $\mathrm{C}$ & -3.56378478 & -1.16014130 & 0.08028314 \\
\hline $\mathrm{C}$ & -3.61046945 & 0.93900869 & -1.17691302 \\
\hline
\end{tabular}




$\begin{array}{lrrr}\text { C } & -4.80340488 & -0.90968192 & 0.59469420 \\ \text { H } & -3.04773909 & -2.06955794 & 0.35310333 \\ \text { C } & -4.84858060 & 1.21339648 & -0.67851267 \\ \text { H } & -3.13729787 & 1.63145338 & -1.85794655 \\ \text { C } & -5.46993292 & 0.29132529 & 0.22083308 \\ \text { H } & -5.26952456 & -1.61018659 & 1.26823978 \\ \text { H } & -5.38853202 & 2.11103885 & -0.94001430 \\ \text { C } & -7.40962414 & -0.18599202 & 1.56978326 \\ \text { H } & -6.85871771 & -0.28846607 & 2.50061042 \\ \text { H } & -8.33491056 & 0.35169194 & 1.73126323 \\ \text { H } & -7.60312351 & -1.15403263 & 1.11631594 \\ \text { O } & -6.65580136 & 0.64424304 & 0.64643703\end{array}$


Total Energy $=-694.674757$

\begin{tabular}{|c|c|c|c|}
\hline Atom & $\mathrm{X}$ & $\mathrm{Y}$ & Z \\
\hline $\mathrm{C}$ & -3.06422473 & -1.29634259 & -0.10337453 \\
\hline $\mathrm{C}$ & -1.70573033 & -1.52452570 & -0.30417471 \\
\hline$C$ & -0.76209184 & -0.49877567 & -0.26455519 \\
\hline $\mathrm{C}$ & -1.20876513 & 0.80675127 & -0.00436939 \\
\hline $\mathrm{C}$ & -2.56770585 & 1.04131154 & 884 \\
\hline $\mathrm{C}$ & -3.49927527 & 0.00548140 & 0.14659453 \\
\hline $\mathrm{H}$ & -3.75589491 & -2.12310938 & -0.14363334 \\
\hline $\mathrm{H}$ & -1.37445427 & -2.53747653 & -0.49831635 \\
\hline $\mathrm{H}$ & -2.92196751 & 2.04505806 & 0.39262463 \\
\hline $\mathrm{C}$ & -5.79547970 & -0.66542368 & 0.31195669 \\
\hline $\mathrm{H}$ & -6.74403145 & -0.17088611 & 20425 \\
\hline $\mathrm{H}$ & -5.82272206 & -1.14713647 & -0.66684868 \\
\hline $\mathrm{H}$ & -5.62203815 & -1.41744152 & 1.08348693 \\
\hline 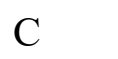 & -0.23166225 & 1.96067214 & 0.09998051 \\
\hline $\mathrm{H}$ & -0.66798493 & 2.85121652 & -0.35583709 \\
\hline $\mathrm{H}$ & -0.09406979 & 2.20305869 & 1.15887913 \\
\hline $\mathrm{C}$ & 0.69947668 & -0.80408043 & -0.53061795 \\
\hline $\mathrm{H}$ & 0.84421609 & -0.94512986 & -1.60760963 \\
\hline $\mathrm{H}$ & 0.96619185 & -1.75512654 & -0.06608724 \\
\hline $\mathrm{C}$ & 1.13443106 & 1.66177908 & -0.52014167 \\
\hline $\mathrm{H}$ & 1.83989345 & 2.44947597 & -0.25277868 \\
\hline $\mathrm{H}$ & 1.05914830 & 1.66061495 & -1.61188182 \\
\hline$C$ & 1.66691082 & 0.29500500 & -0.05471869 \\
\hline $\mathrm{H}$ & 1.67544030 & 0.29561039 & 1.04016558 \\
\hline $\mathrm{O}$ & -4.80482250 & 0.35997733 & 0.35665631 \\
\hline 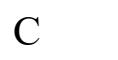 & 3.06517621 & 0.03475184 & -0.53887848 \\
\hline $\mathrm{H}$ & 3.22458122 & 0.00160820 & -1.61254612 \\
\hline $\mathrm{C}$ & 4.10596014 & -0.14584290 & 0.26962812 \\
\hline $\mathrm{H}$ & 4.00856311 & -0.12863493 & 1.35081993 \\
\hline $\mathrm{O}$ & 5.37347217 & -0.34604904 & -0.20153159 \\
\hline $\mathrm{C}$ & 6.33800645 & -0.70500042 & 0.79111511 \\
\hline$U$ & 7.29315958 & -0.78415263 & 0.28078742 \\
\hline U & 6.40340439 & 0.06134668 & 1.56569112 \\
\hline & 6.08921006 & -1.66488694 & 1.24722301 \\
\hline
\end{tabular}


$20^{\circ+}$

Total Energy $=-694.463151$

Atom $\mathrm{X}$

C $\quad-2.97906217 \quad-1.35071051$

$\mathrm{Z}$

C $\quad-1.64198438 \quad-1.51895474$

$-0.13120040$

C $\quad-0.73999416$

$-0.42534243$

$-0.36246191$

C $\quad-1.21638059$

0.87767709

$-0.35460378$

C $\quad-2.55794409$

1.04981838

$-0.01309338$

C $\quad-3.45666513$

$-0.04318329$

0.21711515

$\mathrm{H} \quad-3.65273627$

$-2.19094661$

0.15425985

$\mathrm{H} \quad-1.25979633$

$-2.50490749$

$-0.17041143$

$\mathrm{H} \quad-2.96363477$

2.02082238

$-0.58574414$

C $\quad-5.73830347$

$-0.78098475$

0.46405597

$\mathrm{H} \quad-6.66554372$

$-0.26082649$

0.37389965

$\mathrm{H}$

$-5.77773427$

$-1.25103172$

0.57944011

$\mathrm{H}$

$-5.53620613$

$-1.51431060$

$-0.60558950$

C $\quad-0.25581282$

2.03190351

1.15064289

$\mathrm{H} \quad-0.69866056$

2.93582260

0.15652886

$\mathrm{H} \quad-0.16392725$

2.21605980

$-0.26262687$

C $\quad 0.68236584$

$-0.64204622$

1.23127459

$\mathrm{H} \quad 0.76972646$

$-0.52658049$

$-0.73586943$

$\mathrm{H}$

0.97729528

$-1.66915667$

$-1.82326993$

C $\quad 1.13669471$

1.79451253

$-0.51728722$

H 1.82097182

2.54846472

$-0.43153437$

$\mathrm{H} \quad 1.11564777$

1.90984614

$-0.04462037$

C $\quad 1.65247300$

0.38690438

$-1.51786159$

$\mathrm{H} \quad 1.60951444$

0.25451030

$-0.08695434$

O $\quad-4.72142331$

0.24871963

0.99741259

C $\quad 3.04824413$

0.13419918

0.38929114

H $\quad 3.26936922$

0.24676509

$-0.54378445$

C $\quad 4.03191873$

$-0.24202257$

$-1.59985953$

$\mathrm{H} \quad 3.86637722$

$-0.38440857$

0.28538463

O $\quad 5.28600052$

$-0.46449282$

1.34847314

C $\quad 6.24782094$

$-0.82892510$

$-0.15627329$

$\mathrm{H} \quad 7.18473444$

$-0.99039033$

0.84635278

H $\quad 6.36416828$

$-0.02466202$

0.32410376

$\mathrm{H}$

5.94359350

$-1.74595737$

1.57260880

1.35088265 
21

Atom X

C $\quad 0.67944774$

C $\quad 1.55050398$

C $\quad 1.19034102$

C 2.89450002

C $\quad 2.53127113$

C $\quad 3.38804963$

H $\quad 3.52650829$

$\mathrm{H} \quad 2.93681361$

C $\quad-1.67389111$

$\mathrm{H} \quad-1.26021178$

$\mathrm{H} \quad-1.62241634$

C $\quad-0.77406645$

$\mathrm{H} \quad-0.85037692$

$\mathrm{H} \quad-1.17299901$

C

$\mathrm{H}$

C

$\mathrm{H}$

C

$\mathrm{H}$

$\mathrm{H}$

$\mathrm{H}$

$\mathrm{O}$

$\mathrm{H}$

$\mathrm{C}$

$\mathrm{H}$

$\mathrm{H}$

$\mathrm{H}$

$\mathrm{O}$

C

$\mathrm{H}$

$\mathrm{H}$

$\mathrm{H}$

$-3.10331207$

$-3.30887699$

$-4.11981505$

$-3.97918829$

$-6.39389773$

$-6.41598950$

$-7.35126869$

$-6.20114179$

$-5.40910188$

1.17447306

5.59760037

6.55013011

5.70523227

5.28194613

4.68250005

0.32071131

$-0.53774585$

$-0.07306866$

0.88633799

Total Energy $=-580.145284$

Y

$-0.20197162$

$-1.28438644$

1.08769406

$-1.14262361$

1.23214871

0.13247140

$-2.01501084$

2.21547902

$-0.65102321$

$-1.48381839$

0.22737731

$-0.43914806$

$-1.32476563$

0.39204955

$-0.93907188$

$-1.83270424$

$-0.15064967$

0.75665428

0.44080470

0.33211590

0.13776464

1.48354686

$-0.41903304$

$-2.27883924$

$-0.67778227$

$-0.23926146$

$-1.20013168$

$-1.38420716$

0.41128995

2.31853070

2.26472261

2.44804251

3.21297397
Z

0.55944928

0.46712302

0.29884556

0.12272750

$-0.04292321$

$-0.13647927$

0.06759991

$-0.24171931$

$-0.33807534$

$-0.91458089$

$-0.98430404$

0.90356084

1.53767933

1.48599604

0.01679803

0.59734956

$-0.32285176$

$-0.90220935$

$-0.53630382$

$-1.62210170$

$-0.12323122$

$-0.27741672$

0.04288474

0.67411816

$-0.58974135$

$-0.87071037$

0.36230954

$-1.35924804$

$-0.48135904$

0.39778021

$-0.27284851$

1.40776026

0.14389355 
$21^{\cdot+}$

Atom $\mathrm{X}$

C $\quad 0.64664421$

C $\quad 1.54197381$

C 1.12065429

C $\quad 2.86240215$

C 2.44377096

C $\quad 3.32794864$

$\mathrm{H} \quad 3.52311195$

H 2.84247419

C $\quad-1.62858296$

H $\quad-1.19457471$

$\mathrm{H} \quad-1.51052134$

C $\quad-0.76452680$

$\mathrm{H} \quad-0.83949114$

$\mathrm{H} \quad-1.23105418$

C $\quad-3.04558161$

$\mathrm{H} \quad-3.32183351$

C $\quad-4.01299549$

$\mathrm{H} \quad-3.79819346$

C $\quad-6.24848950$

H $\quad-6.28755675$

$\mathrm{H} \quad-7.20325157$

$\mathrm{H} \quad-5.98779927$

O $\quad-5.28461462$

$\mathrm{H}$

C

$\mathrm{H}$

$\mathrm{H}$

$\mathrm{H}$

$\mathrm{O}$

C

$\mathrm{H}$

$\mathrm{H}$

$\mathrm{H}$

Total Energy $=\mathbf{- 5 7 9 . 9 2 8 7 4 6}$

Y Z

$\begin{array}{ll}-0.22921092 & 0.57478459\end{array}$

$\begin{array}{ll}-1.31522711 & 0.45448498\end{array}$

$\begin{array}{ll}1.09293111 & 0.30753641\end{array}$

$\begin{array}{ll}-1.14333958 & 0.12099565\end{array}$

$\begin{array}{ll}1.26474300 & -0.02671803\end{array}$

$0.16736988 \quad-0.12565313$

$\begin{array}{ll}-1.99210275 & 0.05407320\end{array}$

$\begin{array}{ll}2.25005773 & -0.22090262\end{array}$

$\begin{array}{ll}-0.84325436 & -0.37984971\end{array}$

$\begin{array}{ll}-1.74694168 & -0.80438963\end{array}$

$-0.03443528 \quad-1.09807881$

$\begin{array}{ll}-0.49919793 & 0.93132561\end{array}$

$-1.36105874 \quad 1.59121912$

$0.35143913 \quad 1.41730388$

$\begin{array}{ll}-1.05163214 & -0.03289916\end{array}$

$\begin{array}{ll}-1.93827216 & 0.52484915\end{array}$

$-0.16023456-0.34663928$

$\begin{array}{ll}0.75554061 & -0.88799567\end{array}$

$\begin{array}{ll}0.58958284 & -0.47565721\end{array}$

$\begin{array}{ll}0.58578514 & -1.56362319\end{array}$

$\begin{array}{ll}0.27923658 & -0.06708312\end{array}$

$\begin{array}{ll}1.58088463 & -0.10871304\end{array}$

$-0.37865073-0.01411145$

$\begin{array}{lll}1.17421950 & -2.31383357 & 0.64626150\end{array}$

$\begin{array}{lll}5.56625885 & -0.57416300 & -0.59322959\end{array}$

$\begin{array}{lll}6.48212782 & -0.06366550 & -0.86582914\end{array}$

$\begin{array}{lll}5.69576058 & -1.09591394 & 0.35259912\end{array}$

$\begin{array}{lll}5.27663873 & -1.26882230 & -1.37877728\end{array}$

$\begin{array}{lll}4.57872862 & 0.46865565 & -0.45838411\end{array}$

$\begin{array}{lll}0.20963283 & 2.28563293 & 0.40587125\end{array}$

$\begin{array}{llll}-0.65820680 & 2.18242867 & -0.24619006\end{array}$

$\begin{array}{lll}-0.16500650 & 2.41077493 & 1.42302693\end{array}$

$\begin{array}{lll}0.73558816 & 3.19485819 & 0.12609983\end{array}$ 
22

Atom $\mathrm{X}$

C $\quad 3.85891207$

C $\quad 2.50523251$

C $\quad 1.60332610$

C $\quad 2.08129112$

C $\quad 3.44754683$

C $\quad 4.33499641$

$\mathrm{H} \quad 4.53683468$

$\mathrm{H}$

$\mathrm{H}$

C

$\mathrm{H}$

$\mathrm{H}$

C

2.13407685

3.81565261

1.14230616

1.62582722

0.97699534

0.14075519

$\mathrm{H} \quad 0.03114448$

$\mathrm{H} \quad-0.17087358$

C $\quad-0.21119462$

$\mathrm{H} \quad-0.89808279$

$\mathrm{H} \quad-0.09794252$

C $\quad-0.80586291$

$\mathrm{H} \quad-0.85500740$

C $\quad-2.19309064$

$\mathrm{H} \quad-2.31446103$

C $\quad-3.26724871$

$\mathrm{H} \quad-3.20782588$

O $\quad-4.52264271$

C $\quad-5.53322575$

$\mathrm{H} \quad-6.47203403$

$\mathrm{H} \quad-5.59919082$

$\mathrm{H} \quad-5.33356045$

$\mathrm{H} \quad 5.38712626$
Total Energy $=-656.565084$ Y Z

$-1.46764235 \quad-0.11181223$

$-1.66291557 \quad 0.12866897$

$-0.59263910 \quad 0.13962473$

$0.70202897 \quad-0.10493807$

$0.88868404-0.34378539$

$-0.17925346 \quad-0.34835754$

$-2.31051049$

$-0.11519918$

$-2.66391614$

0.31234252

1.89069830

$-0.52823818$

$1.89059123-0.14579227$

$2.75252901 \quad 0.31770148$

$2.16782566-1.19225624$

$-0.85499543 \quad 0.44265232$

$-1.02636414 \quad 1.51924359$

$-1.78292221-0.04012440$

$1.62324637 \quad 0.51471971$

$2.44085330 \quad 0.29274762$

$1.59046808 \quad 1.60268036$

$0.28805750 \quad 0.03386189$

$0.31919756-1.05945027$

$0.05880473 \quad 0.56329995$

$0.00410100 \quad 1.64104093$

$-0.07028629-0.21071752$

$-0.02973152-1.29401353$

$-0.24323842 \quad 0.30125937$

$-0.54541063-0.66428700$

$-0.60490202-0.12197573$

$0.24135384-1.41802997$

$-1.50169763-1.15103643$

$-0.01069538 \quad-0.53433295$ 


$\begin{array}{llcc}\text { Atom } & \text { X } & \text { Y } & \text { Z } \\ \text { C } & 3.87577761 & -1.41633508 & -0.17564139 \\ \text { C } & 2.53685114 & -1.64670997 & 0.10671200 \\ \text { C } & 1.61963807 & -0.59184891 & 0.17343303 \\ \text { C } & 2.05807036 & 0.71569185 & -0.06899949 \\ \text { C } & 3.40902884 & 0.93558760 & -0.35716891 \\ \text { C } & 4.31554638 & -0.11399845 & -0.40791387 \\ \text { H } & 4.57071028 & -2.24377556 & -0.21664563 \\ \text { H } & 2.19207616 & -2.65762586 & 0.28373906 \\ \text { H } & 3.74878208 & 1.94665383 & -0.54390833 \\ \text { C } & 1.09457086 & 1.88369698 & -0.07232358 \\ \text { H } & 1.54365545 & 2.73072715 & 0.44985055 \\ \text { H } & 0.94486287 & 2.21288745 & -1.10445510 \\ \text { C } & 0.18023246 & -0.89504983 & 0.52747797 \\ \text { H } & 0.07534548 & -0.98483261 & 1.61183172 \\ \text { H } & -0.10955965 & -1.85604897 & 0.10374796 \\ \text { C } & -0.26135379 & 1.58201612 & 0.56175511 \\ \text { H } & -0.96817630 & 2.37489334 & 0.32462246 \\ \text { H } & -0.17966765 & 1.52567412 & 1.64806012 \\ \text { C } & -0.79029692 & 0.21080656 & 0.03297185 \\ \text { H } & -0.78859092 & 0.23668084 & -1.05767351 \\ \text { C } & -2.14740432 & -0.03527259 & 0.52691242 \\ \text { H } & -2.31439484 & -0.14876212 & 1.59232061 \\ \text { C } & -3.26262891 & -0.11912785 & -0.30473591 \\ \text { H } & -3.19053030 & -0.02653301 & -1.38346553 \\ \text { O } & -4.42212376 & -0.31673590 & 0.22389432 \\ \text { H } & -5.60474702 & -0.40515466 & -0.62274298 \\ & -6.25059102 & 0.41570262 & -0.32771260 \\ \text { H } & -5.32366950 & -0.33886026 & -1.66860913 \\ \text { H.35610291 } & -1.35785978 & -0.39135090 \\ \text { H } & & 0.07915547 & -0.62992382\end{array}$


23

$\begin{array}{llcc}\text { Atom } & \text { X } & \text { Y } & \text { Z } \\ \text { C } & 1.57362123 & -0.30208125 & 0.36900910 \\ \text { C } & 2.38892730 & -1.42045972 & 0.17438277 \\ \text { C } & 2.10873859 & 0.97947869 & 0.13392684 \\ \text { C } & 3.70791873 & -1.29925605 & -0.25086171 \\ \text { C } & 3.43610630 & 1.08765764 & -0.29036528 \\ \text { C } & 4.23517042 & -0.03453656 & -0.48600046 \\ \text { H } & 4.31612915 & -2.18249543 & -0.39202225 \\ \text { H } & 3.84979043 & 2.07224300 & -0.46845574 \\ \text { C } & -0.83725485 & -0.64779124 & -0.39843664 \\ \text { H } & -0.48364373 & -1.47357952 & -1.02285990 \\ \text { H } & -0.79392864 & 0.24933501 & -1.01889573 \\ \text { C } & 0.13692113 & -0.50349864 & 0.79611683 \\ \text { H } & 0.06943765 & -1.40640318 & 1.40610562 \\ \text { H } & -0.19860863 & 0.32192864 & 1.42410803 \\ \text { C } & -2.25265759 & -0.90383702 & 0.02915225 \\ \text { H } & -2.45286695 & -1.80720714 & 0.59621752 \\ \text { C } & -3.26184854 & -0.07781973 & -0.23436780 \\ \text { H } & -3.12524786 & 0.84057969 & -0.79698266 \\ \text { C } & -5.52530360 & 0.58368749 & -0.30874708 \\ \text { H } & -5.60852339 & 0.50572403 & -1.39419931 \\ \text { H } & -6.46775902 & 0.29643668 & 0.14759741 \\ \text { H } & -5.28802159 & 1.61293023 & -0.03346242 \\ \text { O } & -4.53689858 & -0.31943616 & 0.19355884 \\ \text { H } & 1.97979147 & -2.40521360 & 0.36443160 \\ \text { C } & 1.29110134 & 2.23216895 & 0.34235953 \\ \text { H } & 0.39008725 & 2.23926852 & -0.27239531 \\ \text { H } & 0.96803745 & 2.33230232 & 1.38047357 \\ & 1.87260610 & 3.11685065 & 0.08935662 \\ & 5.25926631 & 0.08051407 & -0.81449788\end{array}$

Total Energy $=-542.035407$ 


\begin{tabular}{|c|c|c|c|}
\hline & $23^{\circ+}$ & \multicolumn{2}{|c|}{ Total Energy $=-541.819410$} \\
\hline Atom & $X$ & $\mathrm{Y}$ & Z \\
\hline $\mathrm{C}$ & 1.54124256 & -0.30264845 & 0.36022890 \\
\hline $\mathrm{C}$ & 2.38663155 & -1.40715942 & 0.20604833 \\
\hline $\mathrm{C}$ & 2.04233772 & 0.99351167 & 0.11803506 \\
\hline $\mathrm{C}$ & 3.71375067 & -1.25388138 & -0.16947248 \\
\hline $\mathrm{C}$ & 3.38063094 & 1.12929681 & -0.25737516 \\
\hline $\mathrm{C}$ & 4.21299641 & 0.02469387 & -0.40404324 \\
\hline $\mathrm{H}$ & 4.35252776 & -2.11980634 & -0.27363752 \\
\hline $\mathrm{H}$ & 3.77557619 & 2.12046117 & -0.43765512 \\
\hline $\mathrm{C}$ & -0.81498457 & -0.76196128 & -0.49960696 \\
\hline $\mathrm{H}$ & -0.44145957 & -1.65862804 & -0.99617761 \\
\hline $\mathrm{H}$ & -0.72189461 & 0.08320103 & -1.17746284 \\
\hline $\mathrm{C}$ & 0.11423506 & -0.53314186 & 0.77123429 \\
\hline $\mathrm{H}$ & 0.03783260 & -1.41815019 & 1.39967580 \\
\hline $\mathrm{H}$ & -0.27974507 & 0.30904532 & 1.33320029 \\
\hline $\mathrm{C}$ & -2.20023269 & -0.97839966 & -0.08349117 \\
\hline $\mathrm{H}$ & -2.46426733 & -1.88756410 & 0.44314560 \\
\hline $\mathrm{C}$ & -3.21078295 & -0.04582366 & -0.28806671 \\
\hline $\mathrm{H}$ & -3.03302461 & 0.90140983 & -0.78683954 \\
\hline $\mathrm{C}$ & -5.48854933 & 0.62903404 & -0.11924450 \\
\hline $\mathrm{H}$ & -6.19648895 & 0.12788570 & -0.77184116 \\
\hline $\mathrm{H}$ & -5.93607897 & 0.82370552 & 0.84960687 \\
\hline $\mathrm{H}$ & -5.10318827 & 1.53701494 & -0.57155592 \\
\hline $\mathrm{O}$ & -4.40747871 & -0.31666498 & 0.11780446 \\
\hline $\mathrm{H}$ & 1.99586045 & -2.39895167 & 0.39517574 \\
\hline $\mathrm{C}$ & 1.18225184 & 2.22368066 & 0.26904591 \\
\hline $\mathrm{H}$ & 0.30580755 & 2.19390229 & -0.38006287 \\
\hline $\mathrm{H}$ & 0.81880802 & 2.33613414 & 1.29225769 \\
\hline $\mathrm{H}$ & 1.74796640 & 3.11846212 & 0.01835150 \\
\hline $\mathrm{H}$ & 5.24443482 & 0.16199005 & -0.69837018 \\
\hline
\end{tabular}


$24^{\cdot+}$

Atom $\mathrm{X}$

C $\quad-3.97646619$

C $\quad-2.66636549$

C $\quad-1.61580579$

C $\quad-1.93062374$

C $\quad-3.24645949$

C $\quad-4.28554082$

$\mathrm{H} \quad-4.75821357$

H $\quad-2.40691306$

$\mathrm{H} \quad-3.53477363$

C $\quad-6.66540248$

$\mathrm{H} \quad-7.51243238$

H $\quad-6.68762936$

H $\quad-6.64363208$

C $\quad-0.82711555$

H $\quad-1.16164746$

$\mathrm{H} \quad-0.66668940$

C $\quad-0.21006027$

$\mathrm{H} \quad-0.11102752$

$\mathrm{H}$

C $\quad 0.48969533$

$\mathrm{H} \quad 1.27175684$

H $\quad 0.39701721$

C $\quad 0.87854802$

$\mathrm{H} \quad 0.92400140$

O $\quad-5.50262089$

C $\quad 2.24167168$

C $\quad 3.50835245$

C $\quad 2.87828685$

$\mathrm{H} \quad 2.23687785$

C $\quad 4.26722828$

H $\quad 3.38264299$

$\mathrm{H} \quad 2.55828363$

H 2.75920889

$\mathrm{H} \quad 4.73807445$

Total Energy $=-812.346341$ $\mathrm{Y}$

$\mathrm{Z}$

$-1.18692152 \quad-0.21820740$

$-1.53270539-0.35766972$

$-0.58782899$

$-0.17677271$

0.76152470

0.18356737

1.11531439

0.31499136

0.16407172

0.11799432

$-1.91454064$

$-0.36233840$

$-2.54891513$

$-0.61869329$

2.12511454

0.57035059

$-0.22473398$

0.08624040

0.42393402

0.26884475

$-0.60394131$

$-0.93186893$

$-1.03574621$

0.80882494

1.76033078

0.42677151

2.74905382

0.11383607

1.82046085

1.50796086

$-1.02054626$

$-0.42259134$

$-1.13210373$

$-1.51366067$

$-2.03170678$

$-0.03098943$

1.37963704

$-0.25287332$

2.06635969

0.06769378

1.49005595

$-1.33695178$

$-0.06358539$

0.08370877

$-0.15506153$

1.17453205

0.62596591

0.26685531

$-0.45499930$

$-0.47616403$

0.23241069

0.06580724

$-1.81583289$

$-0.07704417$

$-0.35766130$

$-1.56491261$

$-1.12163724$

$-0.02858198$

0.53144526

1.11484180

$-2.13256273$

0.91709720

$-2.64815873$

$-0.76693127$

$-1.18179916$ 


$\begin{array}{llrr}\mathrm{C} & 5.24704817 & -1.52631231 & 1.06036188 \\ \mathrm{H} & 5.54712517 & -2.56985933 & 0.94528976 \\ \mathrm{H} & 6.15279960 & -0.91853485 & 1.03604904 \\ \mathrm{H} & 4.79736812 & -1.41888221 & 2.04943287 \\ \mathrm{C} & 5.09101451 & 1.98933185 & -0.15271068 \\ \mathrm{H} & 5.96789083 & 1.33725318 & -0.16440336 \\ \mathrm{H} & 5.29029791 & 2.86016054 & -0.77274825 \\ \mathrm{H} & 4.90600135 & 2.31526685 & 0.87566735 \\ \mathrm{O} & 3.94764619 & 1.33969180 & -0.69636733\end{array}$


$26^{++}$

Atom $\mathrm{X}$

C $\quad-4.72075465$

C $\quad-3.39882428$

C $\quad-2.37430159$

C $\quad-2.69099222$

C $\quad-4.02660225$

C $\quad-5.03758333$

H $\quad-5.49691906$

H $\quad-3.15067996$

$\mathrm{H} \quad-4.27150047$

C $\quad-1.61467735$

$\mathrm{H} \quad-1.97586504$

$\mathrm{H} \quad-1.43651648$

C $\quad-0.95181755$

$\mathrm{H} \quad-0.84440800$

$\mathrm{H}$

C

$-0.77683509$

$-0.29147651$

$\mathrm{H} \quad 0.47841044$

$\mathrm{H}$

C

$-0.38672986$

0.10440837

$\mathrm{H} \quad 0.13291855$

C $\quad 1.49716653$

C $\quad 2.63206927$

C $\quad 2.01830999$

H 1.52130362

C $\quad 3.48134624$

$\mathrm{H}$

$\mathrm{H}$

$\mathrm{H}$

$\mathrm{H}$

$\mathrm{C}$

$\mathrm{H}$

$\mathrm{H}$

$\mathrm{H}$

C

Total Energy $=-697.799290$

Y Z

$-1.15206759-0.05269440$

$-1.48229210 \quad-0.31872054$

$-0.53704435-0.19761508$

$0.76608992 \quad 0.20680995$

$1.08869997 \quad 0.47156839$

$0.14565161 \quad 0.34467808$

$-1.89852854-0.15295520$

$-2.49066576 \quad-0.62637331$

$2.09724762 \quad 0.78136867$

$1.81593200 \quad 0.39334409$

$2.77864922 \quad 0.02878506$

$1.94819533 \quad 1.46515657$

$-0.94117186$

$-0.97485231$

$-0.53635542$

$-1.62591240$

$-1.95795289$

$-0.18272538$

1.46212998

$-0.28975968$

2.16409184

0.03351154

1.57392851

$-1.37333321$

0.01624685

0.03095452

$-0.10168065$

1.11881455

$-0.34553559$

$-0.52244858$

0.43824418

0.07690955

$-1.77125097$

$-0.18130904$

$-0.19507193$

$-1.60201317$

$-1.46425298$

$-0.08365950$

0.50169154

1.15809598

0.77006545

$-2.12146212$

$-0.95817666$

$-1.02339330$

1.06395148

0.90548351

$-2.90470381$

1.11833543

$-1.81588278$

2.01255430

$2.16701075 \quad-0.01820381$ 
$\begin{array}{llll}\mathrm{H} & 5.21032612 & 1.67975726 & -0.20909992\end{array}$

$\mathrm{H} \quad 4.21449114 \quad 3.12886206 \quad-0.51460650$

$\mathrm{H} \quad 4.07354567 \quad 2.26580342 \quad 1.04752236$

$\begin{array}{llll}\text { O } & 3.21279646 & 1.35732637 & -0.63118398\end{array}$

$\begin{array}{llll}\mathrm{H} & -6.06315634 & 0.41822002 & 0.55366739\end{array}$ 


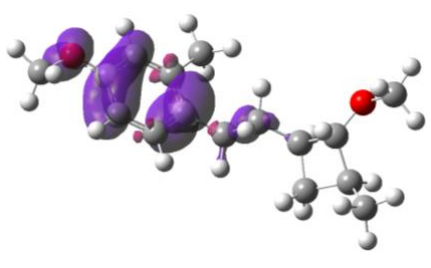

$28^{\circ+}$

Total Energy $=-774.236427$

$\begin{array}{llcc}\text { Atom } & \text { X } & \text { Y } & \text { Z } \\ \text { C } & 2.94901715 & -1.15892427 & -1.20638503 \\ \text { C } & 4.28437473 & -0.83059706 & -0.48117466 \\ \text { C } & 3.46268802 & 0.08950455 & 0.46711916 \\ \text { C } & 2.32883037 & 0.11630694 & -0.57440880 \\ \text { H } & 2.97237750 & -1.22216284 & -2.29170994 \\ \text { H } & 2.49348856 & -2.06701182 & -0.80793888 \\ \text { H } & 4.89982245 & -0.19710470 & -1.12274960 \\ \mathrm{H} & 3.16345845 & -0.45806984 & 1.37005123 \\ \mathrm{H} & 2.49065129 & 0.97116820 & -1.23391026 \\ \mathrm{C} & -1.52619135 & 0.03110498 & -0.74655066 \\ \mathrm{C} & -2.10244337 & -1.21257967 & -0.36436114 \\ \mathrm{C} & -2.28988641 & 1.24041549 & -0.59399836 \\ \mathrm{C} & -3.36450039 & -1.30243383 & 0.14414518 \\ \mathrm{H} & -1.51524077 & -2.11115573 & -0.48960815 \\ \mathrm{C} & -3.55575708 & 1.15148762 & -0.08208330 \\ \mathrm{C} & -4.11424514 & -0.10147607 & 0.29677597 \\ \mathrm{H} & -3.78196295 & -2.25656796 & 0.42100012 \\ \mathrm{H} & -4.17094916 & 2.02952151 & 0.04965623 \\ \mathrm{C} & 0.89304527 & 0.10324454 & -0.07286775 \\ \mathrm{H} & 4.54588531 & 0.83775551 & 2.75019720 \\ \mathrm{H} & 0.73550998 & -0.75756365 & 0.57932962 \\ \mathrm{H} & 0.70260874 & 0.99654939 & 0.52503670 \\ \mathrm{C} & -0.12722199 & 0.04092355 & -1.25083010 \\ \mathrm{H} & 0.06028854 & -0.87248017 & -1.81601476 \\ \mathrm{H} & 0.04608992 & 0.88762761 & -1.91127121 \\ \mathrm{H} & -5.33186365 & -0.04759127 & 0.77802666 \\ \mathrm{H} & -6.02880395 & -1.24474033 & 1.21245308 \\ \mathrm{H} & -6.99392752 & -0.89333963 & 1.55414993 \\ \mathrm{H} & -5.48251962 & -1.71186365 & 2.02734545 \\ \mathrm{H} & 4.93474061 & 1.33304884 & 1.85521169 \\ \mathrm{H} & -1.53162633\end{array}$




$\begin{array}{llrc}\mathrm{H} & 5.17983393 & 2.36478509 & 2.09549344 \\ \mathrm{C} & 5.10453120 & -1.97442330 & 0.09174818 \\ \mathrm{H} & 4.50475730 & -2.58189871 & 0.77227321 \\ \mathrm{H} & 5.46724761 & -2.62891276 & -0.70339341 \\ \mathrm{H} & 5.97360478 & -1.61174280 & 0.64270238 \\ \mathrm{C} & -1.72315455 & 2.57120549 & -0.99632013 \\ \mathrm{H} & -0.78121237 & 2.77292243 & -0.48616965 \\ \mathrm{H} & -1.52193043 & 2.59880753 & -2.06827198 \\ \mathrm{H} & -2.41810296 & 3.37252508 & -0.76021356\end{array}$


$30^{\circ+}$

Atom X

C $\quad-2.14845826$

C $\quad-3.60499653$

C $\quad-2.64436185$

C $\quad-1.57026720$

H $\quad-1.92309029$

$\mathrm{H} \quad-1.78296301$

H $\quad-4.07967903$

$\mathrm{H}$

$\mathrm{H}$

C

C 2.80452808

C 3.10525484

C 4.06318426

$\mathrm{H}$

C $\quad 4.36903861$

C 4.85068689

H 4.42455518

$\mathrm{H}$

4.98660524

$-0.16600743$

$-0.14616526$

0.04298617

0.91937605

C

$\mathrm{H}$

$\mathrm{H}$

$\mathrm{O}$

C

$\mathrm{H}$

$\mathrm{H}$

$\mathrm{H}$

C

0.71060239

0.86155140

$-3.14074047$

$-4.10303108$

$-3.96132921$

$-5.09703349$

$-3.91250211$

$-4.49093073$

$-3.98324658$

$-4.80720450$

$-5.39633188$

Total Energy $=-659.690363$

$\begin{array}{ll}\mathrm{Y} & \mathrm{Z}\end{array}$

$\begin{array}{ll}-1.40699321 & 0.82956882\end{array}$

$\begin{array}{ll}-1.12583953 & 0.62105771\end{array}$

$\begin{array}{ll}0.49604059 & -0.40063875\end{array}$

$\begin{array}{ll}0.00014061 & 0.52198414\end{array}$

$\begin{array}{ll}-1.74185387 & 1.84242354\end{array}$

$\begin{array}{ll}-2.14804665 & 0.12032328\end{array}$

$\begin{array}{ll}-0.49957072 & 1.36909043\end{array}$

$0.04870206-1.38402964$

$0.61318699 \quad 1.42243788$

$-0.36303556$

0.28108718

$-1.46350416$

$-0.42249995$

$\begin{array}{ll}0.79175465 & 0.39806800\end{array}$

$-1.44314111-1.01358262$

$-2.35367329-0.50333921$

$0.79712972 \quad-0.19877623$

$-0.30296400$

$-0.90111371$

$-2.30951123$

$-1.55065903$

$1.68158042 \quad-0.10770683$

$\begin{array}{ll}0.04461981 & -0.10325528\end{array}$

$-0.57290724$

$-1.00286840$

$\begin{array}{ll}1.06918673 & -0.41537635\end{array}$

$\begin{array}{ll}-0.43871724 & 0.87767574\end{array}$

$\begin{array}{ll}-1.47155210 & 1.15897839\end{array}$

$\begin{array}{ll}0.15282794 & 1.79155114\end{array}$

$\begin{array}{ll}1.67505530 & -0.21369384\end{array}$

$2.20436295 \quad-1.17202381$

$\begin{array}{ll}1.73238502 & -2.13979775\end{array}$

$\begin{array}{ll}2.01096237 & -0.78034564\end{array}$

$\begin{array}{ll}3.26969805 & -1.22376419\end{array}$

$-1.96183926 \quad-0.21537124$

$\begin{array}{ll}-2.36704051 & -1.08924945\end{array}$

$\begin{array}{ll}-2.81932966 & 0.39679543\end{array}$

$-1.43594262 \quad-0.51211757$ 


$\begin{array}{lrrr}\mathrm{C} & 2.63837938 & 2.00870779 & 1.16082913 \\ \mathrm{H} & 1.70902236 & 2.41418394 & 0.75828928 \\ \mathrm{H} & 2.45631991 & 1.77808979 & 2.21233665 \\ \mathrm{H} & 3.38764941 & 2.79692118 & 1.12010481 \\ \mathrm{H} & 5.83340027 & -0.26907120 & -1.35158082\end{array}$




\section{General Remarks.}

All reagents and solvents were purchased from commercial sources and used without further purification.

Reactions were monitored by thin-layer chromatography (TLC) carried out on silica gel plates, with detection by UV absorption (254 $\mathrm{nm}$ ) and by heating the plates after dipping them in a solution of $12 \mathrm{M}$ molybdo(VI) phosphoric acid n-hydrate in 95\% ethanol. Silica gel (particle size 40-50 $\mu \mathrm{m}$ ) was used for column chromatography. ${ }^{1} \mathrm{H}$ NMR spectra were collected on a $500 \mathrm{MHz}$ NMR spectrometer using the deuterated solvent as an internal deuterium reference. Chemical shift data are given in $\delta$ units calibrated with residual protic solvent. The multiplicity of a signal is indicated as follows: s, singlet; d, doublet; t, triplet; q, quartet; quint, quintet; m, multiplet. ${ }^{13} \mathrm{C}$ NMR spectra were collected at $125 \mathrm{MHz}$ with proton decoupling using the deuterated solvent as an internal carbon reference. Chemical shift data are given in $\delta$ units calibrated with residual solvent. High-resolution mass spectra (HRMS) were collected on electrospray ionization (ESI)-or direct analysis in real time (DART)-time-of-flight (TOF) spectrometers. Structure optimizations of all stationary points and frequency analyses were carried out at the B3LYP level of density functional theory (DFT) with the 6-311G++(2d,2p) basis set in nitromethane (PCM model). No imaginary frequency was

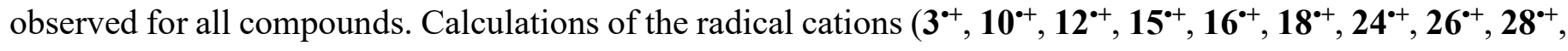
$\mathbf{3 0}^{\circ+}$ ) were carried out with a methyl group instead of the $n$-butyl group for simplification. 


\section{Synthesis and Characterization Data.}

General Procedure for the Synthesis of Enol Ethers (6-9, 21, 23). The enol ethers were synthesized from the respective carboxylic acids or aldehydes according to our previous method. ${ }^{1}$

(1) Okada, Y.; Maeta, N.; Nakayama, K.; Kamiya, H. J. Org. Chem. 2018, 83, 4948-4962.

General Procedure for the $\mathrm{TiO}_{2}$ Photocatalytic Reactions. To a solution of $\mathrm{LiClO}_{4}(1.0 \mathrm{M})$ in $\mathrm{CH}_{3} \mathrm{NO}_{2}(4 \mathrm{~mL})$ stirred at room temperature were added the respective enol ethers $(0.20 \mathrm{mmol})$ and 1-hexene $(503 \mu \mathrm{L}, 4.0$ mmol). The resulting reaction mixture was stirred at room temperature in front of a $15 \mathrm{~W}$ UV lamp (365 nm) until the starting material was consumed (checked by TLC), diluted with water, and extracted with EtOAc. The combined organic layers were dried over $\mathrm{Na}_{2} \mathrm{SO}_{4}$, filtered, and concentrated in vacuo. Yields reported in the manuscript were determined by ${ }^{1} \mathrm{H}$ NMR analysis. Unless otherwise stated, silica gel column chromatography was carried out on $0.40 \mathrm{mmol}$ scale ( 2 batches of the reactions) using Hex/EtOAc $=50 / 1$ as an eluent.

1-(2-(3-Butyl-2-methoxycyclobutyl)ethyl)-4-methoxybenzene (3, all trans). ${ }^{1}$ Colorless oil.

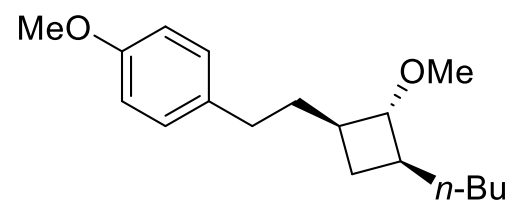

3, all trans

Product yield (1.0 mmol); 71\% (determined by NMR), isolated in 65\% (180 mg, $0.65 \mathrm{mmol})$. 
${ }^{1} \mathrm{H} \mathrm{NMR}\left(\mathrm{CDCl}_{3}, 500 \mathrm{MHz}\right) \delta 7.08(2 \mathrm{H}, \mathrm{d}, J=8.6 \mathrm{~Hz}), 6.81(2 \mathrm{H}, \mathrm{d}, J=8.6 \mathrm{~Hz}), 3.78(3 \mathrm{H}, \mathrm{s}), 3.30(3 \mathrm{H}, \mathrm{s})$, $3.11(1 \mathrm{H}, \mathrm{t}, J=6.8 \mathrm{~Hz}), 2.59-2.43(2 \mathrm{H}, \mathrm{m}), 2.07-1.82(4 \mathrm{H}, \mathrm{m}), 1.65-1.53(2 \mathrm{H}, \mathrm{m}), 1.36-1.17(5 \mathrm{H}, \mathrm{m}), 0.88$ $(3 \mathrm{H}, \mathrm{t}, J=7.1 \mathrm{~Hz}), 0.72(1 \mathrm{H}, \mathrm{q}, J=9.1 \mathrm{~Hz}) ;{ }^{13} \mathrm{C} \mathrm{NMR}\left(125 \mathrm{MHz}, \mathrm{CDCl}_{3}\right) \delta 157.8,134.8,129.4,113.9,86.6$, 56.6, 55.4, 40.7, 40.2, 37.6, 35.1, 33.0, 29.8, 25.6, 22.9, 14.3; HRMS $[\mathrm{M}+\mathrm{H}]^{+}$calcd for $\mathrm{C}_{18} \mathrm{H}_{29} \mathrm{O}_{2} 277.2168$, found 277.2177 .

4-Phenyl-1-butene (5). Colorless oil.

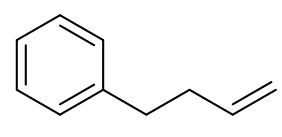

5

Product yield; 68\% (determined by NMR), isolated (pentane) in 43\% (23 mg, $0.17 \mathrm{mmol})$.

${ }^{1} \mathrm{H}$ NMR $\left(\mathrm{CDCl}_{3}, 500 \mathrm{MHz}\right) \delta 7.28(2 \mathrm{H}, \mathrm{t}, J=7.5 \mathrm{~Hz}), 7.21-7.16(3 \mathrm{H}, \mathrm{m}), 5.86(1 \mathrm{H}, \mathrm{ddt}, J=17.2,10.3,6.9$ $\mathrm{Hz}), 5.05(1 \mathrm{H}, \mathrm{dq}, J=17.2,1.7 \mathrm{~Hz}), 4.98(1 \mathrm{H}, \mathrm{dq}, J=10.3,1.7 \mathrm{~Hz}), 2.38(2 \mathrm{H}, \mathrm{q}, J=6.9 \mathrm{~Hz}) ;{ }^{13} \mathrm{C} \mathrm{NMR}(125$ $\left.\mathrm{MHz}, \mathrm{CDCl}_{3}\right) \delta 142.0,138.2,128.6,128.4,126.0,115.1,35.7,35.5 ; \mathrm{HRMS}[\mathrm{M}+\mathrm{H}]^{+}$calcd for $\mathrm{C}_{10} \mathrm{H}_{13}$ 133.1017, found 132.0995 .

1-Methoxy-4-(3-methoxyallyl)benzene (6, cis : trans =1:1). Colorless oil.

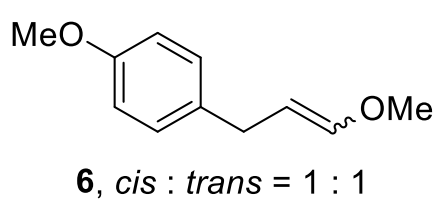

Product yield; isolated in 61\% (1.09 g, $6.09 \mathrm{mmol})$ over 3 steps from 2-(4-methoxyphenyl)acetic acid.

cis: ${ }^{1} \mathrm{H} \mathrm{NMR}\left(\mathrm{CDCl}_{3}, 500 \mathrm{MHz}\right) \delta 7.13(2 \mathrm{H}, \mathrm{d}, J=8.6 \mathrm{~Hz}), 6.82(2 \mathrm{H}, \mathrm{d}, J=8.6 \mathrm{~Hz}), 5.98(1 \mathrm{H}, \mathrm{dt}, J=6.3,1.7$ 
$\mathrm{Hz}), 4.54(1 \mathrm{H}, \mathrm{dt}, J=7.5,6.3 \mathrm{~Hz}), 3.78(3 \mathrm{H}, \mathrm{s}), 3.63(3 \mathrm{H}, \mathrm{s}), 3.35(2 \mathrm{H}, \mathrm{d}, J=7.5 \mathrm{~Hz}) ;{ }^{13} \mathrm{C} \mathrm{NMR}(125 \mathrm{MHz}$, $\left.\mathrm{CDCl}_{3}\right) \delta 157.9,146.6,134.0,129.3,113.9,106.1,59.8,55.4,29.3$.

trans: ${ }^{1} \mathrm{H} \mathrm{NMR}\left(\mathrm{CDCl}_{3}, 500 \mathrm{MHz}\right) \delta 7.12(2 \mathrm{H}, \mathrm{d}, J=8.6 \mathrm{~Hz}), 6.83(2 \mathrm{H}, \mathrm{d}, J=8.6 \mathrm{~Hz}), 6.38(1 \mathrm{H}, \mathrm{dt}, J=12.6$, $1.3 \mathrm{~Hz}), 4.87(1 \mathrm{H}, \mathrm{dt}, J=12.6,7.5 \mathrm{~Hz}), 3.79(3 \mathrm{H}, \mathrm{s}), 3.53(3 \mathrm{H}, \mathrm{s}), 3.21(2 \mathrm{H}, \mathrm{d}, J=6.9 \mathrm{~Hz}) ;{ }^{13} \mathrm{C} \mathrm{NMR}(125$ $\left.\mathrm{MHz}, \mathrm{CDCl}_{3}\right) \delta 158.1,148.0,133.9,129.3,113.9,102.5,56.1,55.4,33.3 ; \mathrm{HRMS}[\mathrm{M}+\mathrm{H}]^{+}$calcd for $\mathrm{C}_{11} \mathrm{H}_{15} \mathrm{O}_{2}$ 179.1072, found 179.1092 .

(3-Methoxyallyl)benzene $(7$, cis $:$ trans $=2: 1)$. Colorless oil.

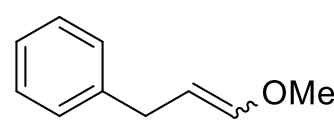

7, cis : trans $=2: 1$

Product yield; isolated in 58\% (860 mg, $5.82 \mathrm{mmol})$ over 4 steps from ethyl 2-phenylacetate.

cis: ${ }^{1} \mathrm{H}$ NMR $\left(\mathrm{CDCl}_{3}, 500 \mathrm{MHz}\right) \delta 7.27(2 \mathrm{H}, \mathrm{t}, J=7.5 \mathrm{~Hz}), 7.24-7.15(3 \mathrm{H}, \mathrm{m}), 6.00(1 \mathrm{H}, \mathrm{dt}, J=6.3,1.3 \mathrm{~Hz})$, $4.56(1 \mathrm{H}, \mathrm{dt}, J=7.5,6.9 \mathrm{~Hz}), 3.64(3 \mathrm{H}, \mathrm{s}), 3.42(2 \mathrm{H}, \mathrm{d}, J=7.5 \mathrm{~Hz}) ;{ }^{13} \mathrm{C} \mathrm{NMR}\left(125 \mathrm{MHz}, \mathrm{CDCl}_{3}\right) \delta 146.8$, $141.8,128.5,128.4,125.9,105.6,59.8,30.3$

trans: ${ }^{1} \mathrm{H} \mathrm{NMR}\left(\mathrm{CDCl}_{3}, 500 \mathrm{MHz}\right) \delta 7.29(2 \mathrm{H}, \mathrm{t}, J=8.0 \mathrm{~Hz}), 7.24-7.15(3 \mathrm{H}, \mathrm{m}), 6.41(1 \mathrm{H}, \mathrm{d}, J=12.6 \mathrm{~Hz})$, $4.89(1 \mathrm{H}, \mathrm{dt}, J=12.6,7.5 \mathrm{~Hz}), 3.53(3 \mathrm{H}, \mathrm{s}), 3.27(2 \mathrm{H}, \mathrm{d}, J=7.5 \mathrm{~Hz}) ;{ }^{13} \mathrm{C} \mathrm{NMR}\left(125 \mathrm{MHz}, \mathrm{CDCl}_{3}\right) \delta 148.2$, 141.8, 128.5, 128.4, 126.1, 102.0, 56.1, 34.2; HRMS $[\mathrm{M}+\mathrm{H}]^{+}$calcd for $\mathrm{C}_{10} \mathrm{H}_{13} \mathrm{O}$ 149.0966, found 149.0953.

1-Methoxy-4-(4-methoxybut-3-en-1-yl)benzene (8, cis : trans =1:1). Colorless oil. 


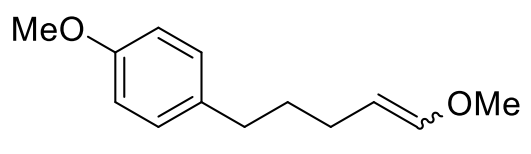

8, cis: trans $=1: 1$

Product yield; isolated in 62\% (1.28 g, $6.21 \mathrm{mmol})$ over 3 steps from 4-(4-methoxyphenyl)butanoic acid.

cis: ${ }^{1} \mathrm{H}$ NMR $\left(\mathrm{CDCl}_{3}, 500 \mathrm{MHz}\right) \delta 7.10(2 \mathrm{H}, \mathrm{d}, J=8.6 \mathrm{~Hz}), 6.81(2 \mathrm{H}, \mathrm{d}, J=8.6 \mathrm{~Hz}), 5.89(1 \mathrm{H}, \mathrm{dt}, J=5.7,1.7$ $\mathrm{Hz}), 4.35(1 \mathrm{H}, \mathrm{dt}, J=6.9,6.3 \mathrm{~Hz}), 3.78(3 \mathrm{H}, \mathrm{s}), 3.58(3 \mathrm{H}, \mathrm{s}), 2.55(2 \mathrm{H}, \mathrm{t}, J=6.3 \mathrm{~Hz}), 2.10(2 \mathrm{H}, \mathrm{dq}, J=7.5$, $1.2 \mathrm{~Hz}), 1.63(2 \mathrm{H}$, quin, $J=7.5 \mathrm{~Hz}) ;{ }^{13} \mathrm{C} \mathrm{NMR}\left(125 \mathrm{MHz}, \mathrm{CDCl}_{3}\right) \delta 157.7,146.4,135.0,129.4,113.8,106.7$, $59.6,55.3,34.8,32.0,23.7$.

trans: ${ }^{1} \mathrm{H} \mathrm{NMR}\left(\mathrm{CDCl}_{3}, 500 \mathrm{MHz}\right) \delta 7.09(2 \mathrm{H}, \mathrm{d}, J=8.6 \mathrm{~Hz}), 6.82(2 \mathrm{H}, \mathrm{d}, J=8.6 \mathrm{~Hz}), 6.28(1 \mathrm{H}, \mathrm{dt}, J=12.6$, $1.1 \mathrm{~Hz}), 4.74(1 \mathrm{H}, \mathrm{dt}, J=12.6,7.5 \mathrm{~Hz}), 3.79(3 \mathrm{H}, \mathrm{s}), 3.50(3 \mathrm{H}, \mathrm{s}), 2.56(2 \mathrm{H}, \mathrm{t}, J=6.3 \mathrm{~Hz}), 1.95(2 \mathrm{H}, \mathrm{q}, J=$ $6.3 \mathrm{~Hz}), 1.63(2 \mathrm{H}$, quin, $J=7.5 \mathrm{~Hz}) ;{ }^{13} \mathrm{C} \mathrm{NMR}\left(125 \mathrm{MHz}, \mathrm{CDCl}_{3}\right) \delta 157.8,147.4,134.7,129.4,113.8,102.8$, 56.0, 55.3, 34.4, 32.8, 27.4; HRMS $[\mathrm{M}+\mathrm{H}]^{+}$calcd for $\mathrm{C}_{13} \mathrm{H}_{19} \mathrm{O}_{2} 207.1385$, found 207.1400.

1-Methoxy-4-(6-methoxyhex-5-en-1-yl)benzene (9, cis : trans =1 : 1). Colorless oil.

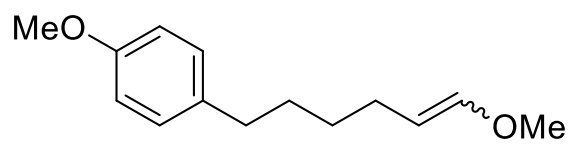

9, cis: trans $=1: 1$

Product yield; isolated in 29\% (639 mg, $2.90 \mathrm{mmol})$ over 5 steps from 4-(4-methoxyphenyl)butanoic acid. cis: ${ }^{1} \mathrm{H} \mathrm{NMR}\left(\mathrm{CDCl}_{3}, 500 \mathrm{MHz}\right) \delta 7.09(2 \mathrm{H}, \mathrm{d}, J=8.6 \mathrm{~Hz}), 6.82(2 \mathrm{H}, \mathrm{d}, J=8.6 \mathrm{~Hz}), 5.86(1 \mathrm{H}, \mathrm{dt}, J=6.3,1.7$ Hz), $4.32(1 \mathrm{H}, \mathrm{q}, J=6.9 \mathrm{~Hz}), 3.78(3 \mathrm{H}, \mathrm{s}), 3.57(3 \mathrm{H}, \mathrm{s}), 2.55(2 \mathrm{H}, \mathrm{t}, J=7.5 \mathrm{~Hz}), 2.08(2 \mathrm{H}, \mathrm{dq}, J=7.5,1.2$ $\mathrm{Hz}), 1.63-1.55(2 \mathrm{H}, \mathrm{m}), 1.37(2 \mathrm{H}$, quin, $J=8.0 \mathrm{~Hz}) ;{ }^{13} \mathrm{C} \mathrm{NMR}\left(125 \mathrm{MHz}, \mathrm{CDCl}_{3}\right) \delta 157.8,146.3,135.0,129.4$, $113.8,107.0,59.6,55.4,35.0,31.2,30.5,27.7$. 
trans: ${ }^{1} \mathrm{H} \mathrm{NMR}\left(\mathrm{CDCl}_{3}, 500 \mathrm{MHz}\right) \delta 7.09(2 \mathrm{H}, \mathrm{d}, J=8.6 \mathrm{~Hz}), 6.81(2 \mathrm{H}, \mathrm{d}, J=8.6 \mathrm{~Hz}), 6.27(1 \mathrm{H}, \mathrm{dt}, J=12.6$, $1.2 \mathrm{~Hz}), 4.71(1 \mathrm{H}, \mathrm{dt}, J=12.0,7.5 \mathrm{~Hz}), 3.78(3 \mathrm{H}, \mathrm{s}), 3.49(3 \mathrm{H}, \mathrm{s}), 2.54(2 \mathrm{H}, \mathrm{t}, J=7.5 \mathrm{~Hz}), 1.94(2 \mathrm{H}, \mathrm{dq}, J=$ 6.9, $1.2 \mathrm{~Hz}), 1.63-1.55(2 \mathrm{H}, \mathrm{m}), 1.37(2 \mathrm{H}$, quin, $J=8.0 \mathrm{~Hz}) ;{ }^{13} \mathrm{C} \mathrm{NMR}\left(125 \mathrm{MHz}, \mathrm{CDCl}_{3}\right) \delta 157.8,147.2$, 135.2, 129.4, 113.8, 103.1, 59.6, 55.4, 35.0, 31.2, 30.5, 27.7; HRMS $[\mathrm{M}+\mathrm{H}]^{+}$calcd for $\mathrm{C}_{14} \mathrm{H}_{21} \mathrm{O}_{2} 221.1542$, found 221.1530 .

1-((3-Butyl-2-methoxycyclobutyl)methyl)-4-methoxybenzene (10, all trans). Colorless oil.

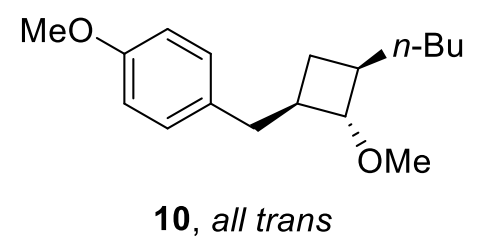

Product yield; $87 \%$ (determined by NMR), isolated in 65\% (68 mg, $0.26 \mathrm{mmol})$.

${ }^{1} \mathrm{H}$ NMR $\left(\mathrm{CDCl}_{3}, 500 \mathrm{MHz}\right) \delta 7.07(2 \mathrm{H}, \mathrm{d}, J=8.6 \mathrm{~Hz}), 6.81(2 \mathrm{H}, \mathrm{d}, J=8.6 \mathrm{~Hz}), 3.78(3 \mathrm{H}, \mathrm{s}), 3.30(3 \mathrm{H}, \mathrm{s})$, $3.11(1 \mathrm{H}, \mathrm{t}, J=6.8 \mathrm{~Hz}), 2.59-2.43(2 \mathrm{H}, \mathrm{m}), 2.07-1.82(4 \mathrm{H}, \mathrm{m}), 1.65-1.53(2 \mathrm{H}, \mathrm{m}), 1.36-1.17(5 \mathrm{H}, \mathrm{m}), 0.88$ $(3 \mathrm{H}, \mathrm{t}, J=7.1 \mathrm{~Hz}), 0.72(1 \mathrm{H}, \mathrm{q}, J=9.1 \mathrm{~Hz}) ;{ }^{13} \mathrm{C} \mathrm{NMR}\left(125 \mathrm{MHz}, \mathrm{CDCl}_{3}\right) \delta 157.8,134.8,129.4,113.9,86.6$, 56.6, 55.4, 40.7, 40.2, 37.6, 35.1, 33.0, 29.8, 25.6, 22.9, 14.3; HRMS $[\mathrm{M}+\mathrm{H}]^{+}$calcd for $\mathrm{C}_{17} \mathrm{H}_{27} \mathrm{O}_{2} 263.2011$, found 263.2020 .

Allylbenzene (13). Colorless oil.

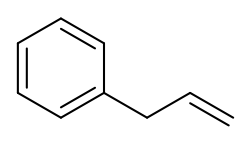

13

Product yield; $68 \%$ (determined by NMR), isolated (pentane) in 28\% (13 mg, $0.11 \mathrm{mmol})$. 
${ }^{1} \mathrm{H}$ NMR $\left(\mathrm{CDCl}_{3}, 500 \mathrm{MHz}\right) \delta$ 7.37-7.15 (5H, m), 5.97 (1H, ddt, $\left.J=17.2,10.3,6.9 \mathrm{~Hz}\right), 5.12-5.04(2 \mathrm{H}, \mathrm{m})$, $3.40(2 \mathrm{H}, \mathrm{d}, J=6.9 \mathrm{~Hz}) ;{ }^{13} \mathrm{C} \mathrm{NMR}\left(125 \mathrm{MHz}, \mathrm{CDCl}_{3}\right) \delta 140.2,137.6,128.7,128.5,126.2,115.9,40.4$; HRMS $[\mathrm{M}+\mathrm{H}]^{+}$calcd for $\mathrm{C}_{9} \mathrm{H}_{11} 119.0861$, found 119.0834 .

1-(3-(3-Butyl-2-methoxycyclobutyl)propyl)-4-methoxybenzene (16, all trans). Colorless oil.

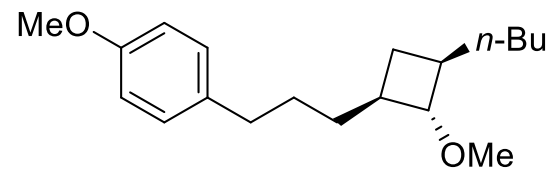

16, all trans

Product yield; 63\% (determined by NMR), isolated in 38\% (44 mg, $0.15 \mathrm{mmol}$ ).

${ }^{1} \mathrm{H} \mathrm{NMR}\left(\mathrm{CDCl}_{3}, 500 \mathrm{MHz}\right) \delta 7.08(2 \mathrm{H}, \mathrm{d}, J=8.6 \mathrm{~Hz}), 6.82(2 \mathrm{H}, \mathrm{d}, J=8.6 \mathrm{~Hz}), 3.78(3 \mathrm{H}, \mathrm{s}), 3.29(3 \mathrm{H}, \mathrm{s})$, $3.06(1 \mathrm{H}, \mathrm{t}, J=6.8 \mathrm{~Hz}), 2.58-2.48(2 \mathrm{H}, \mathrm{m}), 2.04-1.89(3 \mathrm{H}, \mathrm{m}), 1.63-1.50(4 \mathrm{H}, \mathrm{m}), 1.39-1.18(6 \mathrm{H}, \mathrm{m}), 0.88$ $(3 \mathrm{H}, \mathrm{t}, J=6.9 \mathrm{~Hz}), 0.68(1 \mathrm{H}, \mathrm{q}, J=9.7 \mathrm{~Hz}) ;{ }^{13} \mathrm{C} \mathrm{NMR}\left(125 \mathrm{MHz}, \mathrm{CDCl}_{3}\right) \delta 157.8,135.0,129.4,113.8,86.6$, $56.6,55.4,40.7,40.5,35.2,35.1,34.9,29.8,29.7,25.6,22.9,14.3$; HRMS $[\mathrm{M}+\mathrm{H}]^{+}$calcd for $\mathrm{C}_{19} \mathrm{H}_{31} \mathrm{O}_{2}$ 291.2324, found 291.2317.

1-Methoxy-4-(pent-4-en-1-yl)benzene (17). Colorless oil.

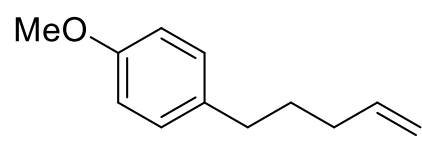

17

Product yield; $21 \%$ (determined by NMR), isolated in 16\% (11 mg, $0.062 \mathrm{mmol}$ ).

${ }^{1} \mathrm{H}$ NMR $\left(\mathrm{CDCl}_{3}, 500 \mathrm{MHz}\right) \delta 7.09(2 \mathrm{H}, \mathrm{d}, J=8.0 \mathrm{~Hz}), 6.83(2 \mathrm{H}, \mathrm{d}, J=8.6 \mathrm{~Hz}), 5.83(1 \mathrm{H}, \mathrm{ddt}, J=17.2,10.3$, $6.3 \mathrm{~Hz}), 5.02(1 \mathrm{H}, \mathrm{dq}, J=17.2,1.7 \mathrm{~Hz}), 4.97(1 \mathrm{H}, \mathrm{d}, J=10.3 \mathrm{~Hz}), 3.79(3 \mathrm{H}, \mathrm{s}), 2.56(2 \mathrm{H}, \mathrm{t}, J=7.5 \mathrm{~Hz}), 2.08$ 
$(2 \mathrm{H}, \mathrm{q}, J=6.9 \mathrm{~Hz}), 1.68(2 \mathrm{H}$, quin, $J=7.5 \mathrm{~Hz}) ;{ }^{13} \mathrm{C} \mathrm{NMR}\left(125 \mathrm{MHz}, \mathrm{CDCl}_{3}\right) \delta 157.8,138.8,134.6,129.4$, 114.8, 113.8, 5.3, 34.5, 33.4, 31.0; HRMS $[\mathrm{M}+\mathrm{H}]^{+}$calcd for $\mathrm{C}_{12} \mathrm{H}_{17} \mathrm{O}$ 177.1279, found 177.1268.

1-(Hex-5-en-1-yl)-4-methoxybenzene (19). Colorless oil.

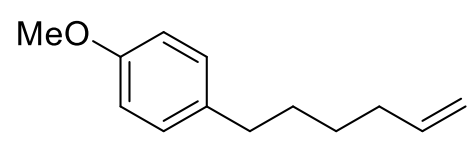

19

Product yield; 67\% (determined by NMR), isolated in 63\% (48 $\mathrm{mg}, 0.25 \mathrm{mmol}$ ).

${ }^{1} \mathrm{H} \mathrm{NMR}\left(\mathrm{CDCl}_{3}, 500 \mathrm{MHz}\right) \delta 7.09(2 \mathrm{H}, \mathrm{d}, J=8.6 \mathrm{~Hz}), 6.82(2 \mathrm{H}, \mathrm{d}, J=8.6 \mathrm{~Hz}), 5.80(1 \mathrm{H}, \mathrm{ddt}, J=16.6,9.7$, $6.9 \mathrm{~Hz}), 4.99(1 \mathrm{H}, \mathrm{dq}, J=17.2,1.7 \mathrm{~Hz}), 4.93(1 \mathrm{H}, \mathrm{d}, J=9.7 \mathrm{~Hz}), 3.79(3 \mathrm{H}, \mathrm{s}), 2.55(2 \mathrm{H}, \mathrm{t}, J=7.5 \mathrm{~Hz}), 2.07$ $(2 \mathrm{H}, \mathrm{ddt}, J=7.5,6.9,1.6 \mathrm{~Hz}), 1.60(2 \mathrm{H}$, quin, $J=7.5 \mathrm{~Hz}), 1.42(2 \mathrm{H}$, quin, $J=8.0 \mathrm{~Hz}) ;{ }^{13} \mathrm{C} \mathrm{NMR}(125 \mathrm{MHz}$, $\left.\mathrm{CDCl}_{3}\right) \delta 157.7,139.0,134.9,129.3,114.4,113.8,55.3,35.0,33.7,31.2,28.6$; HRMS $[\mathrm{M}+\mathrm{H}]^{+}$calcd for $\mathrm{C}_{13} \mathrm{H}_{19} \mathrm{O}$ 191.1436, found 191.1445.

2-(Hydroxymethylene)-6-methoxy-3,4-dihydronaphthalen-1(2H)-one (S3). Orange oil.<smiles>COc1ccc2c(c1)CC/C(=C\O)C2=O</smiles>

S3

To a solution of 6-methoxy-1-tetralone $(\mathbf{S 1}, 5.29 \mathrm{~g}, 30.0 \mathrm{mmol})$ and ethyl formate $(4.82 \mathrm{~mL}, 60.0 \mathrm{mmol})$ in toluene $(60 \mathrm{~mL})$ stirred at $-78^{\circ} \mathrm{C}$ was added $t$-BuOK $(6.73 \mathrm{~g}, 60.0 \mathrm{mmol})$. The resulting reaction mixture was stirred at $-78{ }^{\circ} \mathrm{C}$ to room temperature until the starting material was consumed (checked by TLC), acidified 
with $1 \mathrm{M}$ HClaq. and extracted with EtOAc. The combined organic layers were dried over $\mathrm{Na}_{2} \mathrm{SO}_{4}$, filtered, and concentrated in vacuo. Silica gel column chromatography $(\mathrm{Hex} / \mathrm{EtOAc}=10 / 1)$ gave the titled compound (S3) in 96\% yield (5.86 g, $28.7 \mathrm{mmol})$.

${ }^{1} \mathrm{H} \mathrm{NMR}\left(\mathrm{CDCl}_{3}, 500 \mathrm{MHz}\right) \delta 7.93(2 \mathrm{H}, \mathrm{m}), 6.84(1 \mathrm{H}, \mathrm{dd}, J=8.6,2.9 \mathrm{~Hz}), 6.71(1 \mathrm{H}, \mathrm{d}, J=2.9 \mathrm{~Hz}), 3.84(3 \mathrm{H}$, s), $2.85(2 \mathrm{H}, \mathrm{t}, J=6.3 \mathrm{~Hz}), 2.54(2 \mathrm{H}, \mathrm{t}, J=7.5 \mathrm{~Hz}) ;{ }^{13} \mathrm{C} \mathrm{NMR}\left(125 \mathrm{MHz}, \mathrm{CDCl}_{3}\right) \delta 184.6,172.5,163.5,144.5$, 128.8, 125.1, 113.1, 113.0, 108.2, 55.5, 29.4, 23.3; HRMS $[\mathrm{M}+\mathrm{H}]^{+}$calcd for $\mathrm{C}_{12} \mathrm{H}_{13} \mathrm{O}_{3}$ 205.0865, found 205.0841 .

(6-Methoxy-1,2,3,4-tetrahydronaphthalen-2-yl)methanol (S5). Colorless oil.

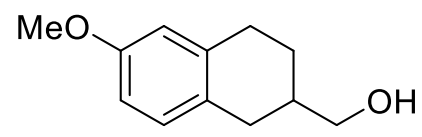

S5

To a solution of the enol $(\mathbf{S 3}, 5.71 \mathrm{~g}, 28.0 \mathrm{mmol})$ in $\mathrm{CH}_{2} \mathrm{Cl}_{2}(60 \mathrm{~mL})$ stirred at $-78{ }^{\circ} \mathrm{C}$ were added borane $t$ butylamine complex $(7.31 \mathrm{~g}, 84.0 \mathrm{mmol})$ and $\mathrm{BF}_{3} \cdot \mathrm{Et}_{2} \mathrm{O}(10.5 \mathrm{~mL}, 84.0 \mathrm{mmol})$. The resulting reaction mixture was stirred at $-78^{\circ} \mathrm{C}$ to room temperature until the starting material was consumed (checked by TLC), acidified with $1 \mathrm{M}$ HClaq. and extracted with $\mathrm{CH}_{2} \mathrm{Cl}_{2}$. The combined organic layers were dried over $\mathrm{Na}_{2} \mathrm{SO}_{4}$, filtered, and concentrated in vacuo. Silica gel column chromatography $(\mathrm{Hex} / \mathrm{EtOAc}=5 / 1)$ gave the titled compound (S5) in 76\% yield (4.09 $\mathrm{g}, 21.3 \mathrm{mmol})$.

${ }^{1} \mathrm{H} \mathrm{NMR}\left(\mathrm{CDCl}_{3}, 500 \mathrm{MHz}\right) \delta 6.99(1 \mathrm{H}, \mathrm{d}, J=8.6 \mathrm{~Hz}), 6.68(1 \mathrm{H}, \mathrm{dd}, J=8.0,2.3 \mathrm{~Hz}), 6.62(1 \mathrm{H}, \mathrm{d}, J=2.3$ $\mathrm{Hz}), 3.77(3 \mathrm{H}, \mathrm{s}), 3.65-3.56(2 \mathrm{H}, \mathrm{m}), 2.85-2.74(3 \mathrm{H}, \mathrm{m}), 2.41(1 \mathrm{H}, \mathrm{dd}, J=15.5,10.3 \mathrm{~Hz}), 2.02-1.89(2 \mathrm{H}, \mathrm{m})$, 1.89-1.84 (1H, br), 1.45-1.35 (1H, m), ${ }^{13} \mathrm{C} \mathrm{NMR}\left(125 \mathrm{MHz}, \mathrm{CDCl}_{3}\right) \delta 157.6,137.9,130.2,128.2,113.5,112.1$, 
67.8, 55.4, 37.4, 31.7, 29.2, 26.0; HRMS $[\mathrm{M}+\mathrm{H}]^{+}$calcd for $\mathrm{C}_{12} \mathrm{H}_{17} \mathrm{O}_{2}$ 193.1229, found 193.1208.

6-Methoxy-1,2,3,4-tetrahydronaphthalene-2-carbaldehyde (S7). Colorless oil.

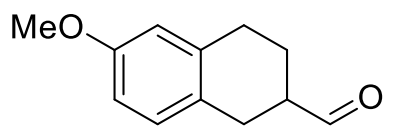

s7

To a solution of $(\mathrm{COCl})_{2}(3.43 \mathrm{~mL}, 40.0 \mathrm{mmol})$ in $\mathrm{CH}_{2} \mathrm{Cl}_{2}(80 \mathrm{~mL})$ stirred at $-78^{\circ} \mathrm{C}$ was added DMSO $(4.26$ $\mathrm{mL}, 60.0 \mathrm{mmol})$. After $5 \mathrm{~min}$, the alcohol $\left(\mathbf{S 5}, 3.85 \mathrm{~g}, 20.0 \mathrm{mmol}\right.$ in $\left.5 \mathrm{~mL} \mathrm{CH}_{2} \mathrm{Cl}_{2}\right)$ was added. After $15 \mathrm{~min}$, $\mathrm{Et}_{3} \mathrm{~N}(16.6 \mathrm{~mL}, 120 \mathrm{mmol})$ was added. The resulting reaction mixture was stirred at $-78{ }^{\circ} \mathrm{C}$ to room temperature until the starting material was consumed (checked by TLC), diluted with water and extracted with $\mathrm{CH}_{2} \mathrm{Cl}_{2}$. The combined organic layers were dried over $\mathrm{Na}_{2} \mathrm{SO}_{4}$, filtered, and concentrated in vacuo. Silica gel column chromatography $(\mathrm{Hex} / \mathrm{EtOAc}=10 / 1)$ gave the titled compound $(\mathbf{S} 7)$ in $79 \%$ yield $(3.01 \mathrm{~g}, 15.8 \mathrm{mmol})$. ${ }^{1} \mathrm{H}$ NMR $\left(\mathrm{CDCl}_{3}, 500 \mathrm{MHz}\right) \delta 9.78(1 \mathrm{H}, \mathrm{d}, J=1.2 \mathrm{~Hz}), 7.05(1 \mathrm{H}, \mathrm{d}, J=8.6 \mathrm{~Hz}), 6.72(1 \mathrm{H}, \mathrm{dd}, J=8.6,2.9$ $\mathrm{Hz}), 6.63(1 \mathrm{H}, \mathrm{d}, J=2.9 \mathrm{~Hz}), 3.77(3 \mathrm{H}, \mathrm{s}), 2.99-2.79(4 \mathrm{H}, \mathrm{m}), 2.71-2.64(1 \mathrm{H}, \mathrm{m}), 2.23-2.16(1 \mathrm{H}, \mathrm{m}), 1.82-$ $1.73(1 \mathrm{H}, \mathrm{m}) ;{ }^{13} \mathrm{C} \mathrm{NMR}\left(125 \mathrm{MHz}, \mathrm{CDCl}_{3}\right) \delta 203.9,157.8,137.0,130.1,126.3,113.4,112.4,55.2,47.0,28.4$, 27.7, 22.8; HRMS [M + H] calcd for $\mathrm{C}_{12} \mathrm{H}_{15} \mathrm{O}_{2}$ 191.1072, found 191.1088.

6-Methoxy-2-(2-methoxyvinyl)-1,2,3,4-tetrahydronaphthalene (20, cis : trans $=1: 2)$. Colorless oil.

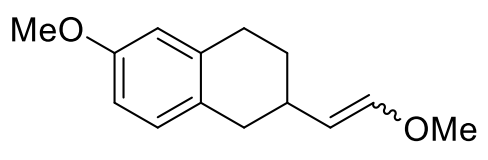

20, cis $:$ trans $=1: 2$ 
To a solution of (methoxymethyl)triphenylphosphonium chloride $(10.3 \mathrm{~g}, 30.0 \mathrm{mmol})$ in THF $(80 \mathrm{~mL})$ stirred at $0{ }^{\circ} \mathrm{C}$ was added $t$-BuOK $(3.37 \mathrm{~g}, 30.0 \mathrm{mmol})$. After the color turned red, the aldehyde $(\mathbf{S} 7,2.85 \mathrm{~g}, 15.0$ mmol) was added, and the reaction was stirred at $0{ }^{\circ} \mathrm{C}$ until the starting material was consumed (checked by TLC), diluted with water, and extracted with EtOAc. The combined organic layers were dried over $\mathrm{Na}_{2} \mathrm{SO}_{4}$, filtered, and concentrated in vacuo. Silica gel column chromatography $(\mathrm{Hex} / \mathrm{EtOAc}=50 / 1)$ gave the titled compound (20) in $80 \%$ yield $(2.62 \mathrm{~g}, 12.6 \mathrm{mmol})$.

cis: ${ }^{1} \mathrm{H} \mathrm{NMR}\left(\mathrm{CDCl}_{3}, 500 \mathrm{MHz}\right) \delta 6.95(1 \mathrm{H}, \mathrm{d}, J=8.0 \mathrm{~Hz}), 6.67(1 \mathrm{H}, \mathrm{dt}, J=8.6,2.9 \mathrm{~Hz}), 6.63-6.60(1 \mathrm{H}, \mathrm{m})$, $5.88(1 \mathrm{H}, \mathrm{d}, J=6.3 \mathrm{~Hz}), 4.34(1 \mathrm{H}, \mathrm{dd}, J=8.6,6.3 \mathrm{~Hz}), 3.76(3 \mathrm{H}, \mathrm{s}), 3.59(3 \mathrm{H}, \mathrm{s}), 2.91-2.85(1 \mathrm{H}, \mathrm{m}), 2.85-$ $2.74(3 \mathrm{H}, \mathrm{m}), 2.54-2.41(1 \mathrm{H}, \mathrm{m}), 1.93-1.86(1 \mathrm{H}, \mathrm{m}), 1.59-1.49(1 \mathrm{H}, \mathrm{m}) ;{ }^{13} \mathrm{C} \mathrm{NMR}\left(125 \mathrm{MHz}, \mathrm{CDCl}_{3}\right) \delta 157.5$, $145.7,137.6,130.0,128.7,113.5,111.9,111.7,59.7,55.3,35.6,30.3,29.8,29.2$.

trans: ${ }^{1} \mathrm{H}$ NMR $\left(\mathrm{CDCl}_{3}, 500 \mathrm{MHz}\right) \delta 6.97(1 \mathrm{H}, \mathrm{d}, J=8.0 \mathrm{~Hz}), 6.67(1 \mathrm{H}, \mathrm{dt}, J=8.6,2.9 \mathrm{~Hz}), 6.63-6.60(1 \mathrm{H}$, m), $6.40(1 \mathrm{H}, \mathrm{d}, J=12.6 \mathrm{~Hz}), 4.80(1 \mathrm{H}, \mathrm{dd}, J=12.6,8.0 \mathrm{~Hz}), 3.77(3 \mathrm{H}, \mathrm{s}), 3.52(3 \mathrm{H}, \mathrm{s}), 2.85-2.74(3 \mathrm{H}, \mathrm{m})$, 2.54-2.41 (1H, m), 2.54-2.41 (1H, m), 2.38-2.30 (1H, m), 1.93-1.86 (1H, m), 1.59-1.49 $(1 \mathrm{H}, \mathrm{m}) ;{ }^{13} \mathrm{C}$ NMR $\left(125 \mathrm{MHz}, \mathrm{CDCl}_{3}\right) \delta 157.6,146.7,137.5,129.9,128.5,113.5,112.0,108.1,55.9,55.3,36.5,34.0,30.9,29.4 ;$ HRMS $[\mathrm{M}+\mathrm{H}]^{+}$calcd for $\mathrm{C}_{14} \mathrm{H}_{19} \mathrm{O}_{2} 219.1385$, found 219.1412 .

4-Methoxy-1-(4-methoxybut-3-en-1-yl)-2-methylbenzene (21, cis : trans =1:1). Colorless oil.

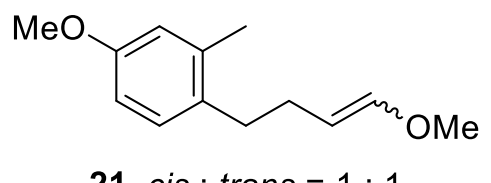

21, cis : trans $=1: 1$

cis: ${ }^{1} \mathrm{H} \mathrm{NMR}\left(\mathrm{CDCl}_{3}, 500 \mathrm{MHz}\right) \delta 7.06(2 \mathrm{H}, \mathrm{d}, J=8.6 \mathrm{~Hz}), 6.71-6.65(2 \mathrm{H}, \mathrm{m}), 5.88(1 \mathrm{H}, \mathrm{dt}, J=6.3,1.6 \mathrm{~Hz})$, 
$4.39(1 \mathrm{H}, \mathrm{dt}, J=7.3,6.3 \mathrm{~Hz}), 3.76(3 \mathrm{H}, \mathrm{s}), 3.56(3 \mathrm{H}, \mathrm{s}), 2.58(2 \mathrm{H}, \mathrm{t}, J=7.5 \mathrm{~Hz}), 2.31(2 \mathrm{H}, \mathrm{dt}, J=8.0,7.5 \mathrm{~Hz})$, $2.29(3 \mathrm{H}, \mathrm{s}) ;{ }^{13} \mathrm{C} \mathrm{NMR}\left(125 \mathrm{MHz}, \mathrm{CDCl}_{3}\right) \delta 157.7,146.5,137.4,132.8,129.7,115.8,110.9,106.3$ 59.6, 55.3, $32.6,24.8,19.7$.

trans: ${ }^{1} \mathrm{H}$ NMR $\left(\mathrm{CDCl}_{3}, 500 \mathrm{MHz}\right) \delta 7.03(2 \mathrm{H}, \mathrm{d}, J=8.6 \mathrm{~Hz}), 6.71-6.65(2 \mathrm{H}, \mathrm{m}), 6.31(1 \mathrm{H}, \mathrm{dt}, J=12.6,1.5$ $\mathrm{Hz}), 4.78(1 \mathrm{H}, \mathrm{dt}, J=12.6,7.5 \mathrm{~Hz}), 3.77(3 \mathrm{H}, \mathrm{s}), 3.50(3 \mathrm{H}, \mathrm{s}), 2.58(2 \mathrm{H}, \mathrm{t}, J=7.5 \mathrm{~Hz}), 2.28(3 \mathrm{H}, \mathrm{s}), 2.15(2 \mathrm{H}$, $\mathrm{dt}, J=8.6,8.0 \mathrm{~Hz}) ;{ }^{13} \mathrm{C} \mathrm{NMR}\left(125 \mathrm{MHz}, \mathrm{CDCl}_{3}\right) \delta 157.8,147.5,137.2,132.5,129.9,115.9,111.1,102.6$, 56.0, 55.3, 34.1, 29.0, 19.8; HRMS $[\mathrm{M}+\mathrm{H}]^{+}$calcd for $\mathrm{C}_{13} \mathrm{H}_{19} \mathrm{O}_{2} 207.1385$, found 207.1411.

2-(Hydroxymethylene)-3,4-dihydronaphthalen-1(2H)-one (S4). Orange oil.<smiles>O=C1/C(=C/O)CCc2ccccc21</smiles>

S4

To a solution of $\alpha$-tetralone (S2, $4.00 \mathrm{~mL}, 30.0 \mathrm{mmol})$ and ethyl formate $(4.82 \mathrm{~mL}, 60.0 \mathrm{mmol})$ in toluene (60 $\mathrm{mL})$ stirred at $-78^{\circ} \mathrm{C}$ was added $t$ - BuOK $(6.73 \mathrm{~g}, 60.0 \mathrm{mmol})$. The resulting reaction mixture was stirred at $78{ }^{\circ} \mathrm{C}$ to room temperature until the starting material was consumed (checked by TLC), acidified with $1 \mathrm{M}$ HClaq. and extracted with EtOAc. The combined organic layers were dried over $\mathrm{Na}_{2} \mathrm{SO}_{4}$, filtered, and concentrated in vacuo. Silica gel column chromatography $(\mathrm{Hex} / \mathrm{EtOAc}=10 / 1)$ gave the titled compound $(\mathbf{S 4})$ in $93 \%$ yield $(4.87 \mathrm{~g}, 28.0 \mathrm{mmol})$.

${ }^{1} \mathrm{H} \mathrm{NMR}\left(\mathrm{CDCl}_{3}, 500 \mathrm{MHz}\right) \delta 8.22(1 \mathrm{H}, \mathrm{d}, J=6.3 \mathrm{~Hz}), 7.97(1 \mathrm{H}, \mathrm{dd}, J=7.5,1.2 \mathrm{~Hz}), 7.45(1 \mathrm{H}, \mathrm{tt}, J=7.5$, $1.2 \mathrm{~Hz}), 7.35(1 \mathrm{H}, \mathrm{t}, J=7.5 \mathrm{~Hz}), 7.23(1 \mathrm{H}, \mathrm{d}, J=7.5 \mathrm{~Hz}), 2.90(2 \mathrm{H}, \mathrm{t}, J=6.9 \mathrm{~Hz}), 2.58(2 \mathrm{H}, \mathrm{t}, J=8.0 \mathrm{~Hz})$; ${ }^{13} \mathrm{C}$ NMR $\left(125 \mathrm{MHz}, \mathrm{CDCl}_{3}\right) \delta 182.4,176.9,141.6,132.9,131.5,128.1,127.0,126.3,108.6,28.8,22.8$; 
HRMS $[\mathrm{M}+\mathrm{H}]^{+}$calcd for $\mathrm{C}_{11} \mathrm{H}_{11} \mathrm{O}_{2}$ 175.0759, found 175.0745 .

(1,2,3,4-Tetrahydronaphthalen-2-yl)methanol (S6). Colorless oil.

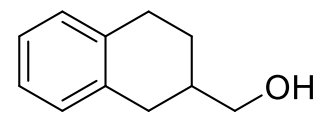

56

To a solution of the enol $(\mathbf{S 4}, 4.87 \mathrm{~g}, 28.0 \mathrm{mmol})$ in $\mathrm{CH}_{2} \mathrm{Cl}_{2}(60 \mathrm{~mL})$ stirred at $-78{ }^{\circ} \mathrm{C}$ were added borane $t$ butylamine complex $(7.31 \mathrm{~g}, 84.0 \mathrm{mmol})$ and $\mathrm{BF}_{3} \cdot \mathrm{Et}_{2} \mathrm{O}(10.5 \mathrm{~mL}, 84.0 \mathrm{mmol})$. The resulting reaction mixture was stirred at $-78^{\circ} \mathrm{C}$ to room temperature until the starting material was consumed (checked by TLC), acidified with $1 \mathrm{M}$ HClaq. and extracted with $\mathrm{CH}_{2} \mathrm{Cl}_{2}$. The combined organic layers were dried over $\mathrm{Na}_{2} \mathrm{SO}_{4}$, filtered, and concentrated in vacuo. Silica gel column chromatography $(\mathrm{Hex} / \mathrm{EtOAc}=5 / 1)$ gave the titled compound (S5) in 53\% yield $(2.40 \mathrm{~g}, 14.8 \mathrm{mmol})$.

${ }^{1} \mathrm{H}$ NMR $\left(\mathrm{CDCl}_{3}, 500 \mathrm{MHz}\right) \delta$ 7.13-7.01 (4H, m), 3.65-3.56 (2H, m), 2.91-2.75 (3H, m), $2.50(1 \mathrm{H}, \mathrm{dd}, J=$ 16.0, $10.3 \mathrm{~Hz}), 2.03-1.94(2 \mathrm{H}, \mathrm{m}), 1.94-1.88(1 \mathrm{H}, \mathrm{br}), 1.47-1.37(1 \mathrm{H}, \mathrm{m}) ;{ }^{13} \mathrm{C} \mathrm{NMR}\left(125 \mathrm{MHz}, \mathrm{CDCl}_{3}\right) \delta$ 136.9, 136.1, 129.4, 129.0, 125.7, 67.8, 37.1, 32.5, 28.9, 26.0; HRMS $[\mathrm{M}+\mathrm{H}]^{+}$calcd for $\mathrm{C}_{11} \mathrm{H}_{15} \mathrm{O}$ 163.1123, found 163.1099 .

1,2,3,4-Tetrahydronaphthalene-2-carbaldehyde (S8). Colorless oil.<smiles>O=CC1CCc2ccccc2C1</smiles>

S8

To a solution of $(\mathrm{COCl})_{2}(2.40 \mathrm{~mL}, 28.0 \mathrm{mmol})$ in $\mathrm{CH}_{2} \mathrm{Cl}_{2}(60 \mathrm{~mL})$ stirred at $-78^{\circ} \mathrm{C}$ was added DMSO $(2.98$ $\mathrm{mL}, 42.0 \mathrm{mmol})$. After $5 \mathrm{~min}$, the alcohol $\left(\mathbf{S 4}, 2.27 \mathrm{~g}, 14.0 \mathrm{mmol}\right.$ in $\left.5 \mathrm{~mL} \mathrm{CH}_{2} \mathrm{Cl}_{2}\right)$ was added. After $15 \mathrm{~min}$, 
$\mathrm{Et}_{3} \mathrm{~N}(11.7 \mathrm{~mL}, 84.0 \mathrm{mmol})$ was added. The resulting reaction mixture was stirred at $-78{ }^{\circ} \mathrm{C}$ to room temperature until the starting material was consumed (checked by TLC), diluted with water and extracted with $\mathrm{CH}_{2} \mathrm{Cl}_{2}$. The combined organic layers were dried over $\mathrm{Na}_{2} \mathrm{SO}_{4}$, filtered, and concentrated in vacuo. Silica gel column chromatography $(\mathrm{Hex} / \mathrm{EtOAc}=10 / 1)$ gave the titled compound $(\mathbf{S 8})$ in 80\% yield $(1.81 \mathrm{~g}, 11.3 \mathrm{mmol})$. ${ }^{1} \mathrm{H} \mathrm{NMR}\left(\mathrm{CDCl}_{3}, 500 \mathrm{MHz}\right) \delta 9.79(1 \mathrm{H}, \mathrm{d}, J=1.2 \mathrm{~Hz}), 7.19-7.07(4 \mathrm{H}, \mathrm{m}), 3.04-2.96(2 \mathrm{H}, \mathrm{m}), 2.95-2.81(2 \mathrm{H}$, m), 2.76-2.67 (1H, m), 2.26-2.19 (1H, m), 1.84-1.75 (1H, m); ${ }^{13} \mathrm{C}$ NMR (125 MHz, $\left.\mathrm{CDCl}_{3}\right) \delta 203.9,136.0$, 134.4, 129.3, 129.0, 126.2, 126.0, 46.9, 28.5, 28.2, 23.0; HRMS $[\mathrm{M}+\mathrm{H}]^{+}$calcd for $\mathrm{C}_{11} \mathrm{H}_{13} \mathrm{O} 161.0966$, found 161.0960.

2-(2-Methoxyvinyl)-1,2,3,4-tetrahydronaphthalene (22, cis : trans $=3: 1)$. Colorless oil.

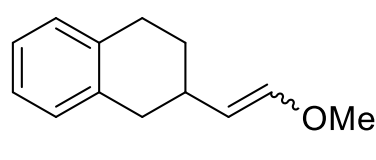

22, cis : trans $=3: 1$

cis: ${ }^{1} \mathrm{H} \mathrm{NMR}\left(\mathrm{CDCl}_{3}, 500 \mathrm{MHz}\right) \delta$ 7.12-7.02 (4H, m), $5.89(1 \mathrm{H}, \mathrm{d}, J=6.3 \mathrm{~Hz}), 4.35(1 \mathrm{H}, \mathrm{dd}, J=7.5,6.3 \mathrm{~Hz})$, $3.60(3 \mathrm{H}, \mathrm{s}), 2.94-2.90(1 \mathrm{H}, \mathrm{m}), 2.90-2.78(3 \mathrm{H}, \mathrm{m}), 2.63-2.49(1 \mathrm{H}, \mathrm{m}), 1.96-1.88(1 \mathrm{H}, \mathrm{m}), 1.61-1.51(1 \mathrm{H}, \mathrm{m})$; ${ }^{13} \mathrm{C}$ NMR $\left(125 \mathrm{MHz}, \mathrm{CDCl}_{3}\right) \delta 145.8,136.7,136.6,129.3,129.0,125.6,125.5,111.7,59.8,36.4,30.2,29.9$, 28.9 .

trans: ${ }^{1} \mathrm{H}$ NMR $\left(\mathrm{CDCl}_{3}, 500 \mathrm{MHz}\right) \delta$ 7.12-7.02 (4H, m), $6.41(1 \mathrm{H}, \mathrm{d}, J=13.2 \mathrm{~Hz}), 4.80(1 \mathrm{H}, \mathrm{dd}, J=12.6,8.0$ Hz), $3.53(3 \mathrm{H}, \mathrm{s}), 2.90-2.78(3 \mathrm{H}, \mathrm{m}), 2.63-2.49(1 \mathrm{H}, \mathrm{m}), 2.41-2.33(1 \mathrm{H}, \mathrm{m}), 1.96-1.88(1 \mathrm{H}, \mathrm{m}), 1.61-1.51(1 \mathrm{H}$, $\mathrm{m}) ;{ }^{13} \mathrm{C} \mathrm{NMR}\left(125 \mathrm{MHz}, \mathrm{CDCl}_{3}\right) \delta 146.7,136.7,136.5,129.3,129.2,125.7,125.6,108.1,56.0,37.4,33.8$, 30.9, 29.1; HRMS $[\mathrm{M}+\mathrm{H}]^{+}$calcd for $\mathrm{C}_{13} \mathrm{H}_{17} \mathrm{O}$ 189.1279, found 189.1303. 
1-(4-Methoxybut-3-en-1-yl)-2-methylbenzene (23, cis : trans =1 : 1). Colorless oil.

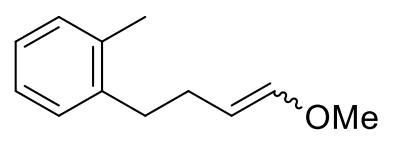

23, cis $:$ trans $=1: 1$

cis: ${ }^{1} \mathrm{H}$ NMR $\left(\mathrm{CDCl}_{3}, 500 \mathrm{MHz}\right) \delta$ 7.18-7.06 (4H, m), $5.89(1 \mathrm{H}, \mathrm{dt}, J=6.3,1.2 \mathrm{~Hz}), 4.41(1 \mathrm{H}, \mathrm{dt}, J=13.8$, $7.5 \mathrm{~Hz}), 3.56(3 \mathrm{H}, \mathrm{s}), 2.64(2 \mathrm{H}, \mathrm{t}, J=6.9 \mathrm{~Hz}), 2.35(2 \mathrm{H}, \mathrm{q}, J=8.0 \mathrm{~Hz}), 2.32(3 \mathrm{H}, \mathrm{s}) ;{ }^{13} \mathrm{C} \mathrm{NMR}(125 \mathrm{MHz}$, $\left.\mathrm{CDCl}_{3}\right) \delta 146.7,140.6,136.2,130.3,128.9,126.1,125.9,106.3,59.6,33.5,24.5,19.5$.

trans: ${ }^{1} \mathrm{H} \mathrm{NMR}\left(\mathrm{CDCl}_{3}, 500 \mathrm{MHz}\right) \delta$ 7.18-7.06 (4H, m), $6.32(1 \mathrm{H}, \mathrm{dt}, J=12.6,1.1 \mathrm{~Hz}), 4.79(1 \mathrm{H}, \mathrm{dt}, J=12.6$, $7.5 \mathrm{~Hz}), 3.50(3 \mathrm{H}, \mathrm{s}), 2.64(2 \mathrm{H}, \mathrm{t}, J=6.9 \mathrm{~Hz}), 2.31(3 \mathrm{H}, \mathrm{s}), 2.20(2 \mathrm{H}, \mathrm{q}, J=8.0 \mathrm{~Hz}) ;{ }^{13} \mathrm{C} \mathrm{NMR}(125 \mathrm{MHz}$, $\left.\mathrm{CDCl}_{3}\right) \delta 147.6,140.3,136.0,130.2,129.0,126.0,125.9,102.5,56.0,35.0,28.7,19.5 ; \mathrm{HRMS}[\mathrm{M}+\mathrm{H}]^{+}$calcd for $\mathrm{C}_{12} \mathrm{H}_{17} \mathrm{O}$ 177.1279, found 177.1293.

2-(3-Butyl-2-methoxycyclobutyl)-6-methoxy-1,2,3,4-tetrahydronaphthalene (24, all trans). Colorless oil.

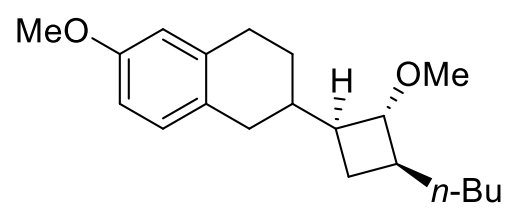

24, all trans

Product yield; $87 \%$ (determined by NMR), isolated in $73 \%$ (87 $\mathrm{mg}, 0.29 \mathrm{mmol})$.

Minor Diastereomer: ${ }^{1} \mathrm{H} \mathrm{NMR}\left(\mathrm{CDCl}_{3}, 500 \mathrm{MHz}\right) \delta 6.99(1 \mathrm{H}, \mathrm{d}, J=8.6 \mathrm{~Hz}), 6.67(1 \mathrm{H}, \mathrm{dd}, J=8.6,2.3 \mathrm{~Hz})$, 6.62-6.59 (1H, m), $3.76(3 \mathrm{H}, \mathrm{s}), 3.34(3 \mathrm{H}, \mathrm{s}), 3.25(1 \mathrm{H}, \mathrm{t}, J=6.9 \mathrm{~Hz}), 2.84-2.68(3 \mathrm{H}, \mathrm{m}), 2.39(1 \mathrm{H}, \mathrm{dd}, J=$ 16.0, $10.9 \mathrm{~Hz}), 2.08-1.82(4 \mathrm{H}, \mathrm{m}), 1.69-1.58(2 \mathrm{H}, \mathrm{m}), 1.43-1.20(6 \mathrm{H}, \mathrm{m}), 0.89(3 \mathrm{H}, \mathrm{t}, J=6.9 \mathrm{~Hz}), 0.82(1 \mathrm{H}$, 
$\mathrm{q}, J=9.7 \mathrm{~Hz}) ;{ }^{13} \mathrm{C} \mathrm{NMR}\left(125 \mathrm{MHz}, \mathrm{CDCl}_{3}\right) \delta 157.6,138.0,130.2,128.9,113.6,112.0,85.2,56.5,55.4,45.4$, $40.4,39.8,35.3,33.4,29.8,29.6,26.9,23.6,22.9,14.3$.

Major Diastereomer: ${ }^{1} \mathrm{H} \mathrm{NMR}\left(\mathrm{CDCl}_{3}, 500 \mathrm{MHz}\right) \delta 6.96(1 \mathrm{H}, \mathrm{d}, J=8.6 \mathrm{~Hz}), 6.67(1 \mathrm{H}, \mathrm{dd}, J=8.6,2.3 \mathrm{~Hz})$, 6.62-6.59 (1H, m), $3.76(3 \mathrm{H}, \mathrm{s}), 3.33(3 \mathrm{H}, \mathrm{s}), 3.25(1 \mathrm{H}, \mathrm{t}, J=6.9 \mathrm{~Hz}), 2.84-2.68(3 \mathrm{H}, \mathrm{m}), 2.29(1 \mathrm{H}, \mathrm{dd}, J=$ 16.0, $10.9 \mathrm{~Hz}), 2.08-1.82(4 \mathrm{H}, \mathrm{m}), 1.69-1.58(2 \mathrm{H}, \mathrm{m}), 1.43-1.20(6 \mathrm{H}, \mathrm{m}), 0.89(3 \mathrm{H}, \mathrm{t}, J=6.9 \mathrm{~Hz}), 0.82(1 \mathrm{H}$, $\mathrm{q}, J=9.7 \mathrm{~Hz}) ;{ }^{13} \mathrm{C} \mathrm{NMR}\left(125 \mathrm{MHz}, \mathrm{CDCl}_{3}\right) \delta 157.6,138.1,130.1,128.7,113.6,112.1,85.2,56.5,55.4,45.4$, 40.6, 39.7, 35.3, 32.9, 29.8, 29.6, 27.2, 23.6, 22.9, 14.3; HRMS $[\mathrm{M}+\mathrm{H}]^{+}$calcd for $\mathrm{C}_{20} \mathrm{H}_{31} \mathrm{O}_{2} 303.2324$, found 303.2323 .

2-Vinyl-1,2,3,4-tetrahydronaphthalene (27). Colorless oil.

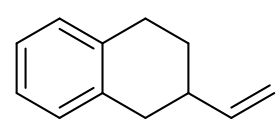

27

Product yield; 58\% (determined by NMR), isolated in 55\% (34 mg, $0.22 \mathrm{mmol}$ ).

${ }^{1} \mathrm{H}$ NMR $\left(\mathrm{CDCl}_{3}, 500 \mathrm{MHz}\right) \delta$ 7.13-7.06 (4H, m), $5.92(1 \mathrm{H}, \mathrm{ddd}, J=17.2,10.3,6.9 \mathrm{~Hz}), 5.09(1 \mathrm{H}, \mathrm{dt}, J=$ 17.2, $1.9 \mathrm{~Hz}), 5.01(1 \mathrm{H}, \mathrm{dq}, J=10.3,1.7 \mathrm{~Hz}), 2.91-2.83(3 \mathrm{H}, \mathrm{m}), 2.67-2.59(1 \mathrm{H}, \mathrm{m}), 2.49-2.40(1 \mathrm{H}, \mathrm{m}), 2.02-$ $1.95(1 \mathrm{H}, \mathrm{m}), 1.62-1.52(1 \mathrm{H}, \mathrm{m}) ;{ }^{13} \mathrm{C} \mathrm{NMR}\left(125 \mathrm{MHz}, \mathrm{CDCl}_{3}\right) \delta 143.4,136.6,136.3,129.3,129.0,125.8$, 125.7, 113.1, 38.4, 35.4, 29.2, 29.0; HRMS [M + H] $]^{+}$calcd for $\mathrm{C}_{12} \mathrm{H}_{15}$ 159.1174, found 159.1150.

1-(2-(3-Butyl-2-methoxycyclobutyl)ethyl)-4-methoxy-2-methylbenzene (28, all trans). Colorless oil. 


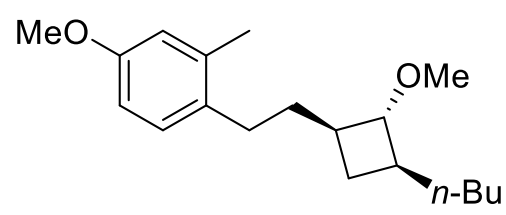

28, all trans

Product yield; $86 \%$ (determined by NMR), isolated in 65\% (76 mg, $0.26 \mathrm{mmol}$ ).

${ }^{1} \mathrm{H}$ NMR $\left(\mathrm{CDCl}_{3}, 500 \mathrm{MHz}\right) \delta 7.02(2 \mathrm{H}, \mathrm{d}, J=8.6 \mathrm{~Hz}), 6.69(1 \mathrm{H}, \mathrm{d}, J=2.3 \mathrm{~Hz}), 6.67(1 \mathrm{H}, \mathrm{dd}, J=8.0,2.3$ $\mathrm{Hz}), 3.77(3 \mathrm{H}, \mathrm{s}), 3.31(3 \mathrm{H}, \mathrm{s}), 3.11(1 \mathrm{H}, \mathrm{t}, J=6.9 \mathrm{~Hz}), 2.54-2.43(2 \mathrm{H}, \mathrm{m}), 2.27(3 \mathrm{H}, \mathrm{s}), 2.10-1.92(3 \mathrm{H}, \mathrm{m})$, 1.86-1.78 (1H, m), 1.62-1.50 (2H, m), 1.37-1.20 (5H, m), $0.89(3 \mathrm{H}, \mathrm{t}, J=6.9 \mathrm{~Hz}), 0.74(1 \mathrm{H}, \mathrm{q}, J=9.7 \mathrm{~Hz})$; ${ }^{13} \mathrm{C}$ NMR $\left(125 \mathrm{MHz}, \mathrm{CDCl}_{3}\right) \delta 157.8,137.2,133.0,129.7,115.9,111.1,86.7,56.6,55.4,40.8,40.6,36.4$, 35.1, 30.4, 29.8, 25.7, 22.9, 19.7, 14.3; HRMS $[\mathrm{M}+\mathrm{H}]^{+}$calcd for $\mathrm{C}_{19} \mathrm{H}_{31} \mathrm{O}_{2}$ 291.2324, found 291.2300.

1-(But-3-en-1-yl)-2-methylbenzene (31). Colorless oil.

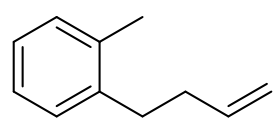

31

Product yield; 74\% (determined by NMR), isolated (pentane) in 70\% (41 mg, $0.28 \mathrm{mmol}$ ).

${ }^{1} \mathrm{H}$ NMR $\left(\mathrm{CDCl}_{3}, 500 \mathrm{MHz}\right) \delta 7.17(4 \mathrm{H}, \mathrm{m}), 5.90(1 \mathrm{H}, \mathrm{ddt}, J=16.6,10.3,6.9 \mathrm{~Hz}), 5.06(1 \mathrm{H}, \mathrm{dq}, J=17.2,1.7$ Hz), $4.99(1 \mathrm{H}, \mathrm{dq}, J=10.3,1.7 \mathrm{~Hz}), 2.69(2 \mathrm{H}, \mathrm{t}, J=8.0 \mathrm{~Hz}), 2.36-2.29(2 \mathrm{H}, \mathrm{m}), 2.31(3 \mathrm{H}, \mathrm{s}) ;{ }^{13} \mathrm{C} \mathrm{NMR}(125$ $\left.\mathrm{MHz}, \mathrm{CDCl}_{3}\right) \delta 140.2,138.5,136.1,130.3,128.9,126.1,126.0,115.0,3.5,32.9,19.5 ; \mathrm{HRMS}[\mathrm{M}+\mathrm{H}]^{+}$calcd for $\mathrm{C}_{11} \mathrm{H}_{15}$ 147.1174, found 147.1177. 

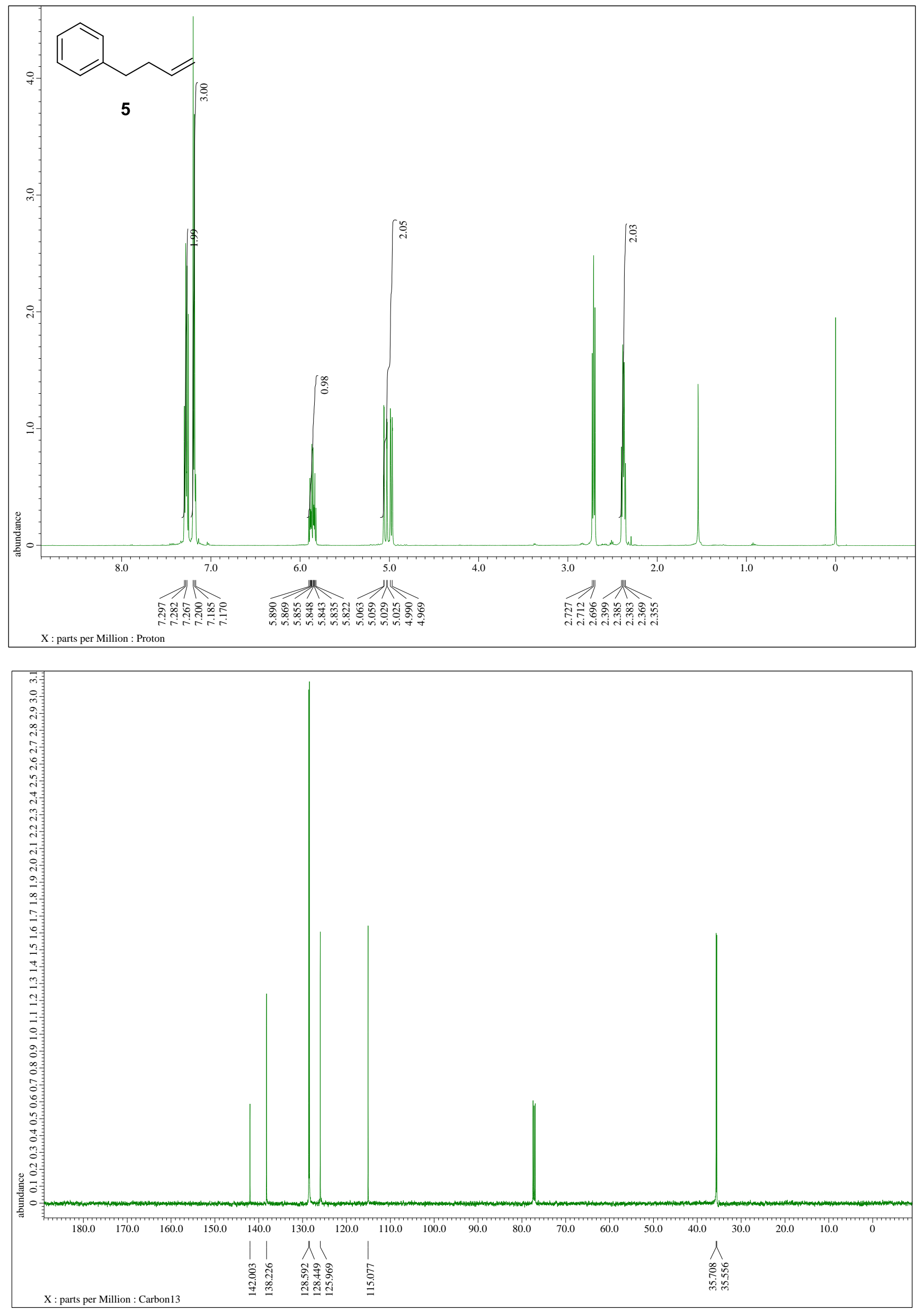

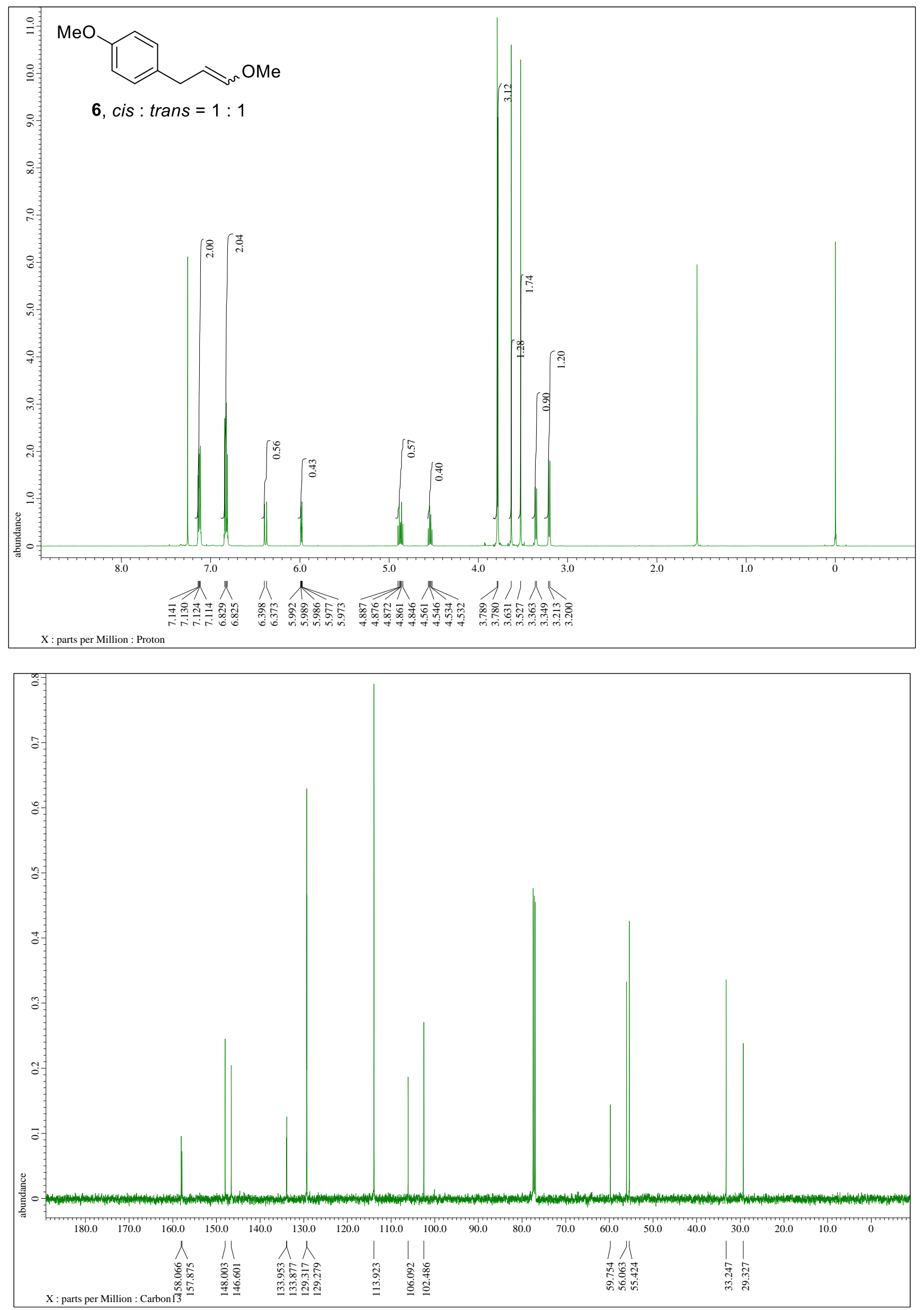

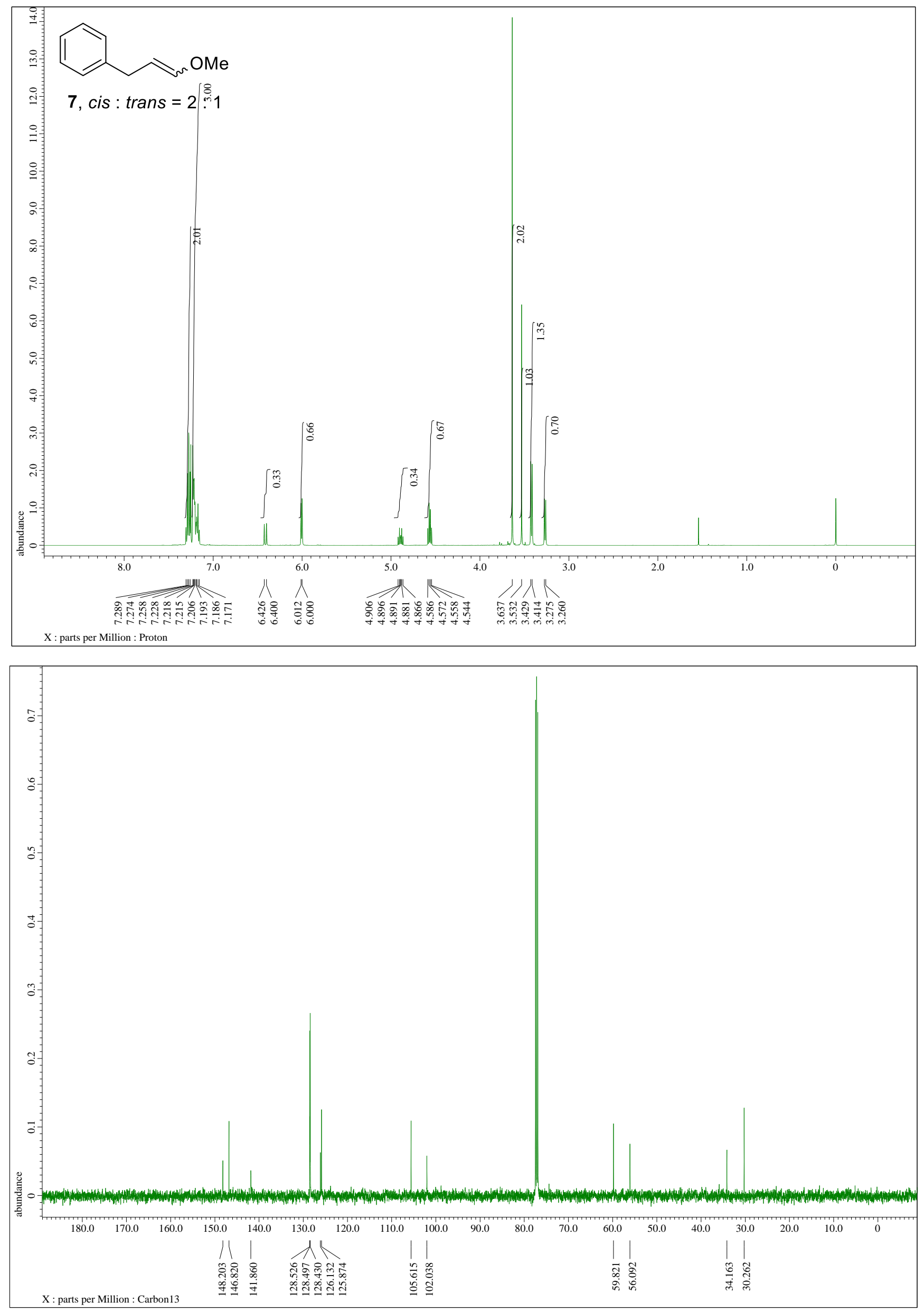

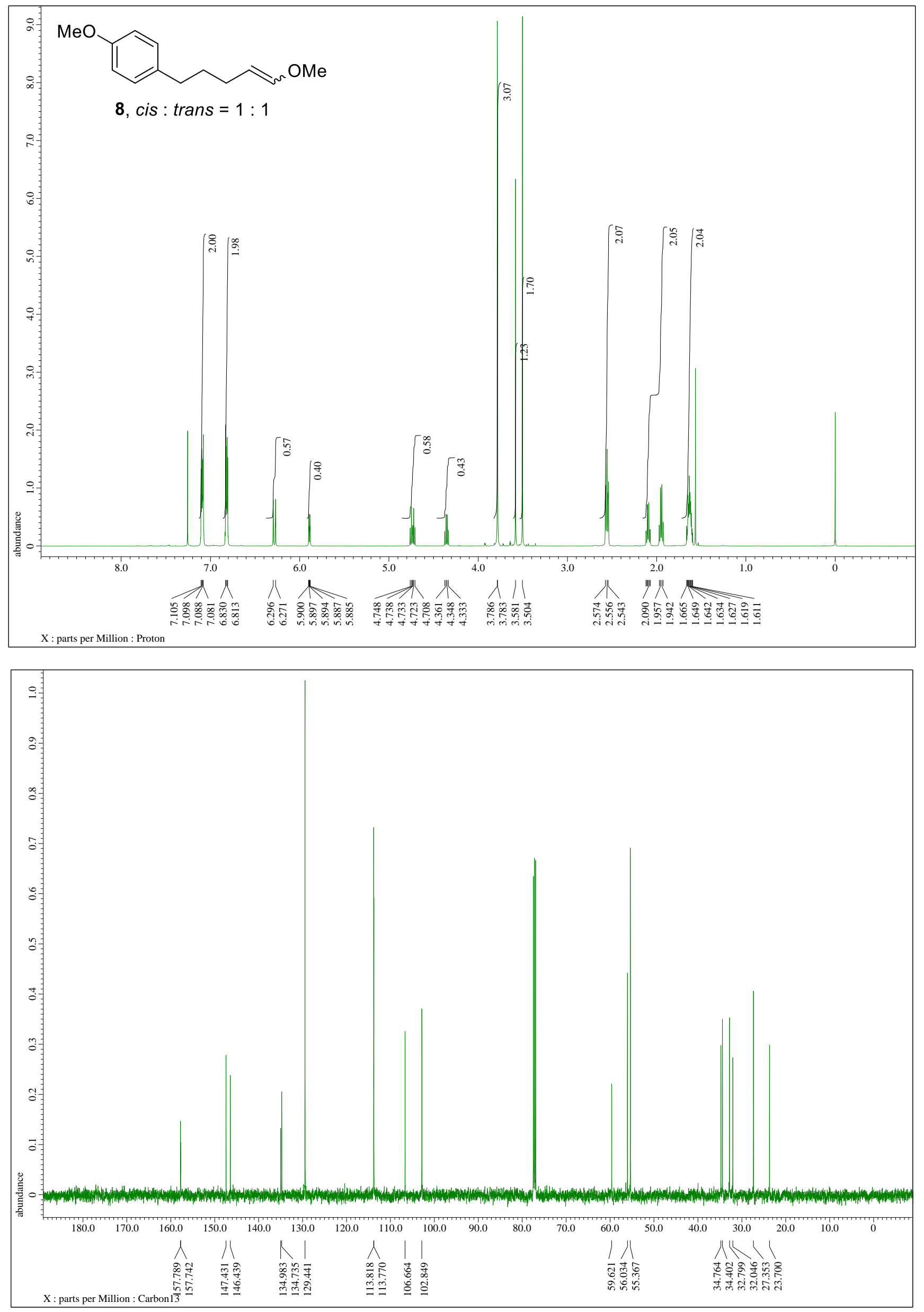

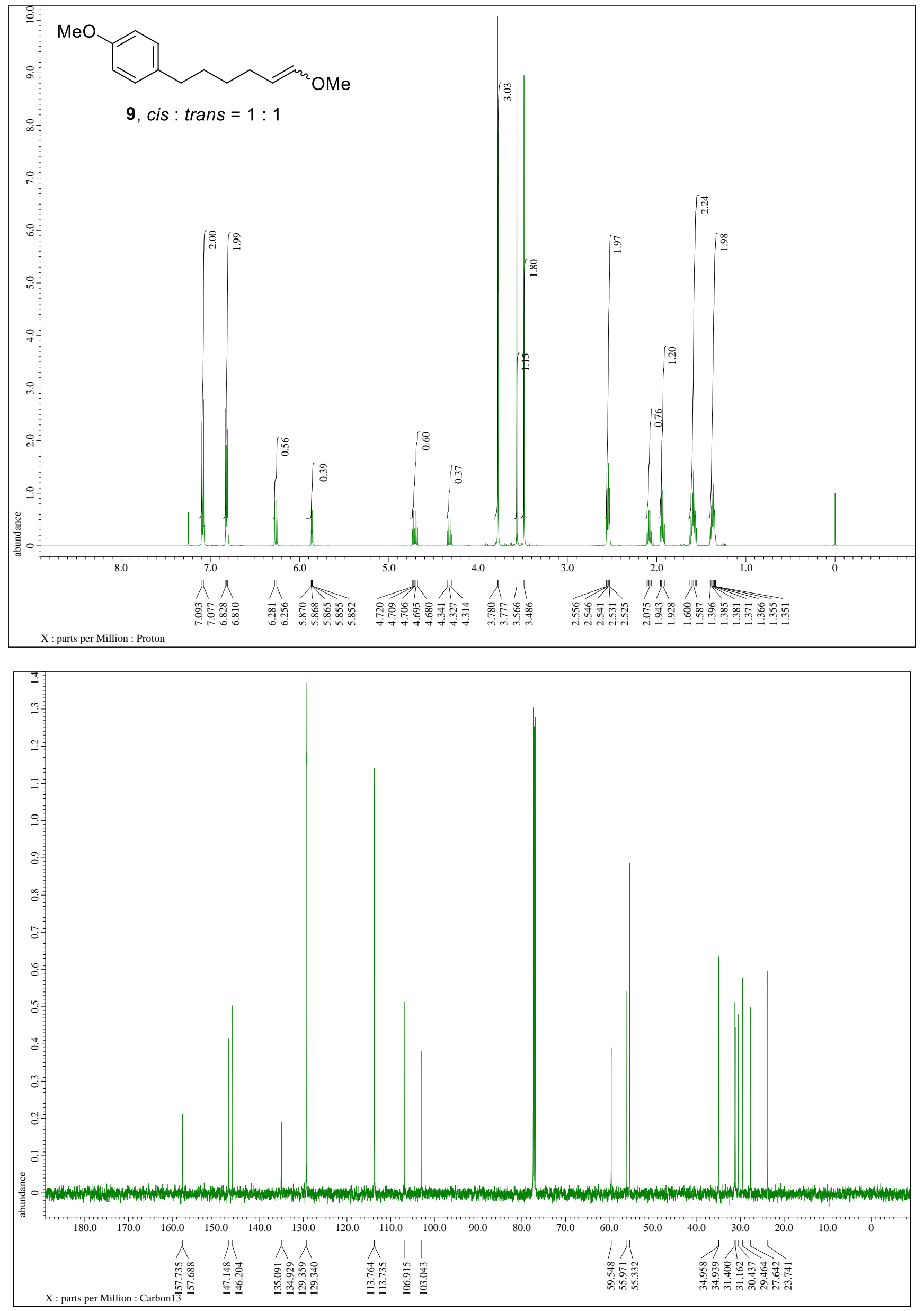

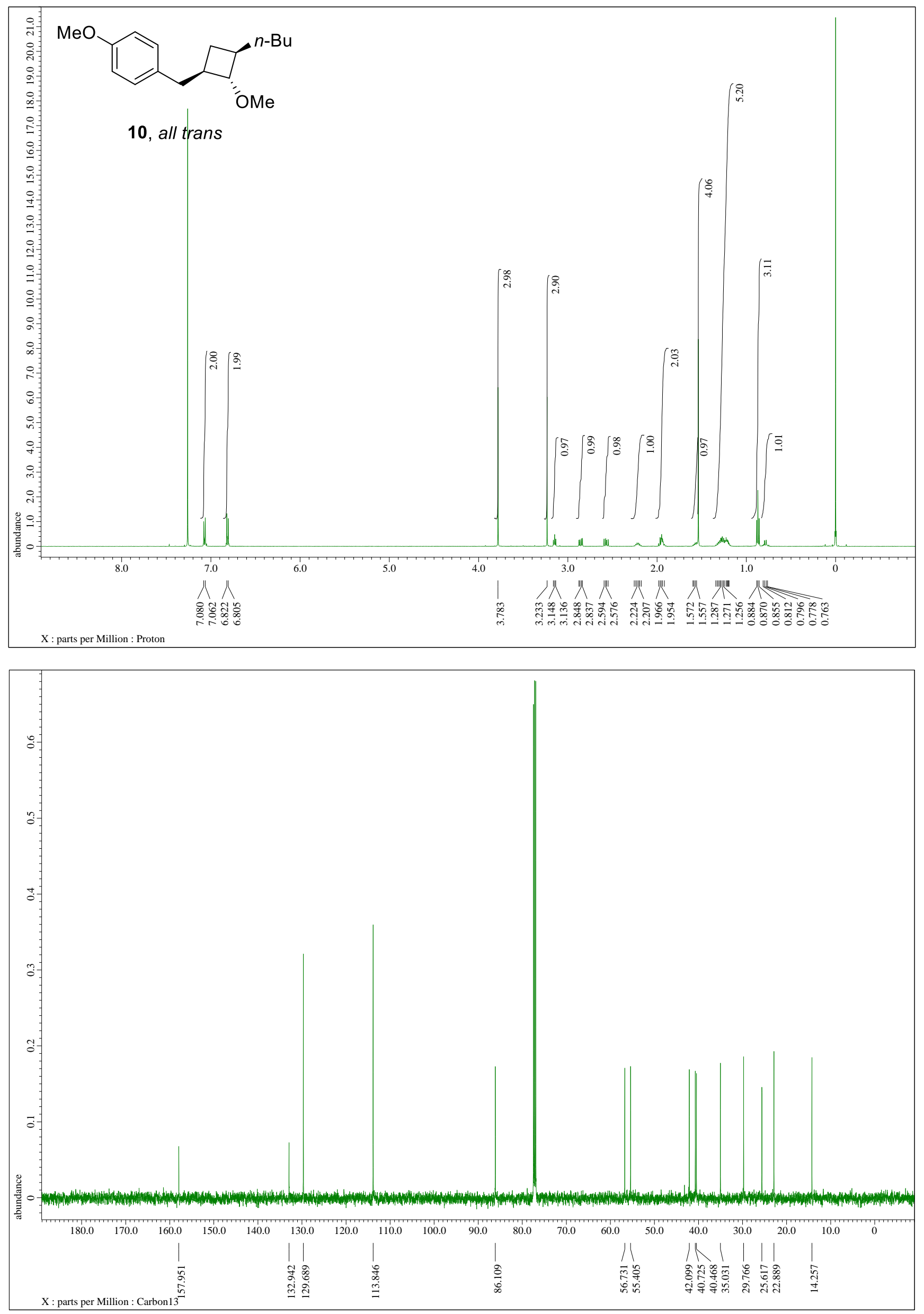

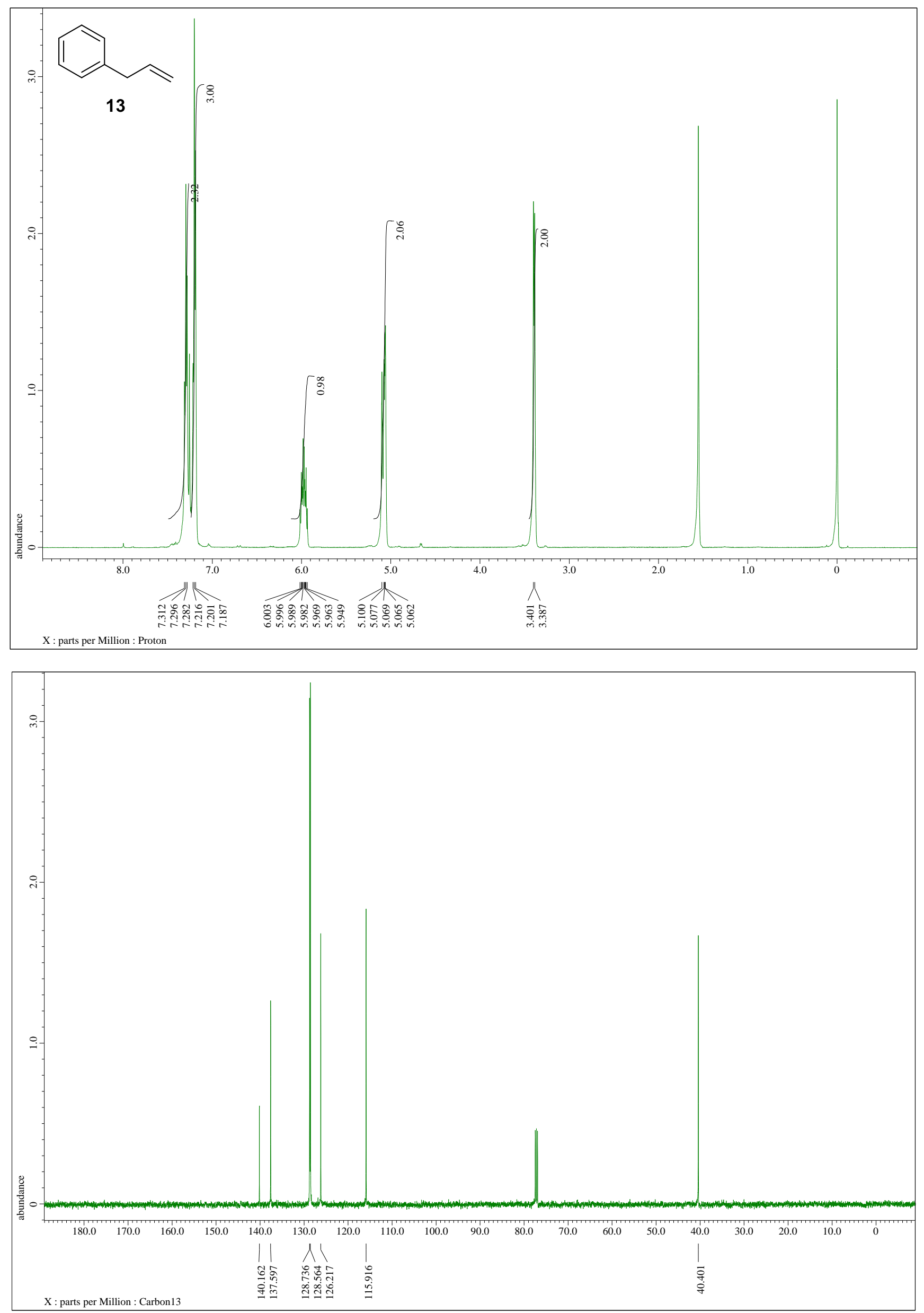

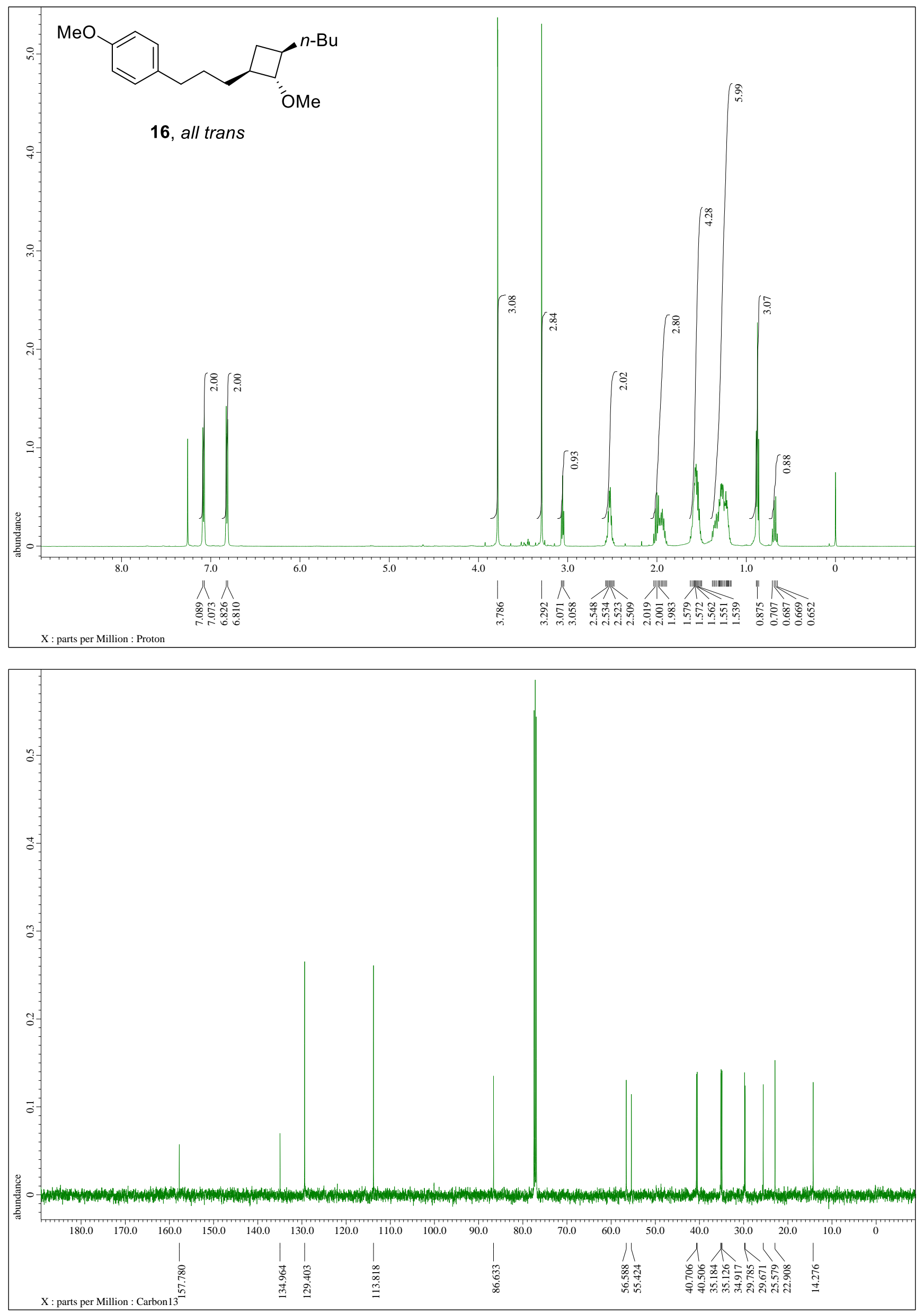

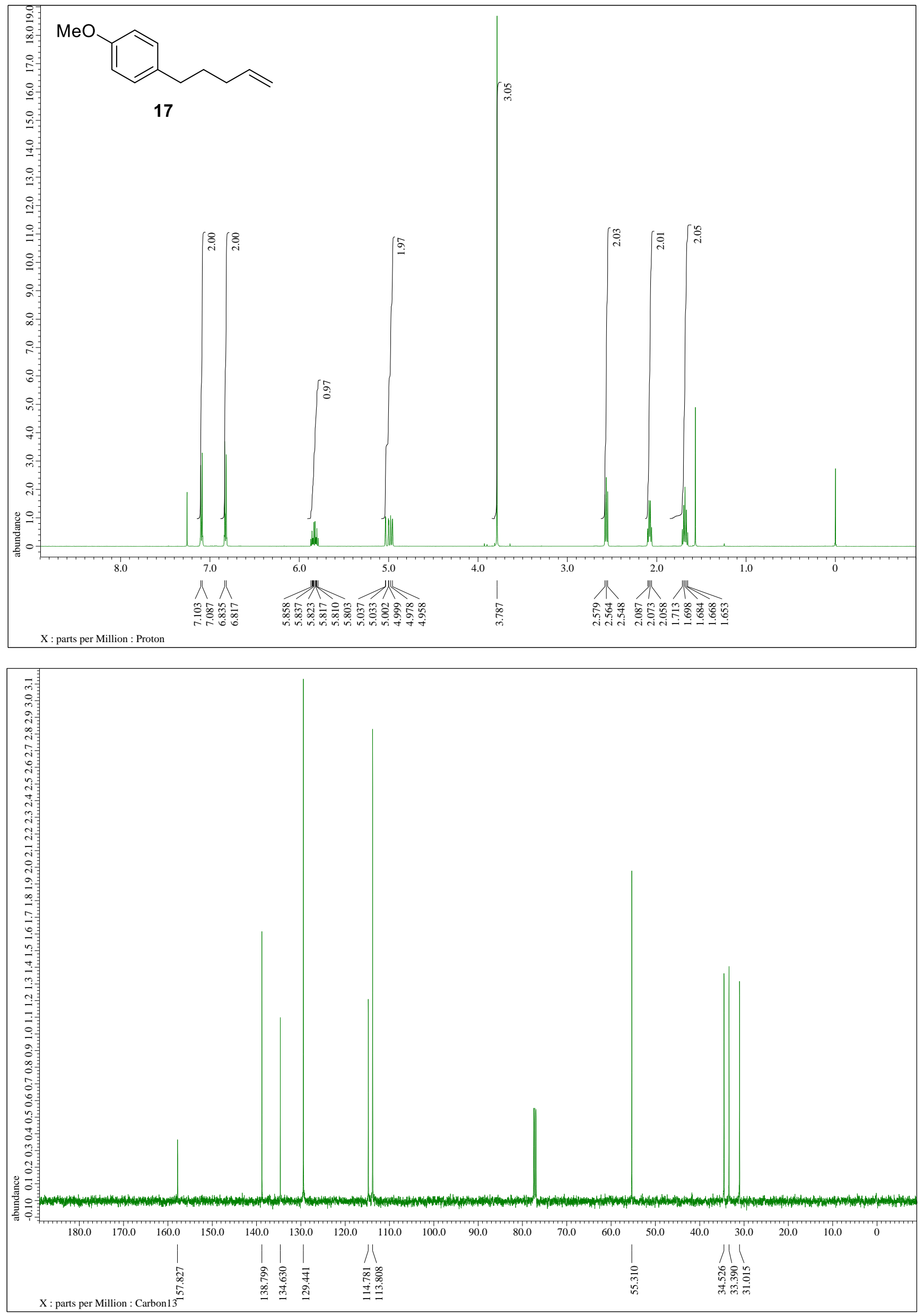

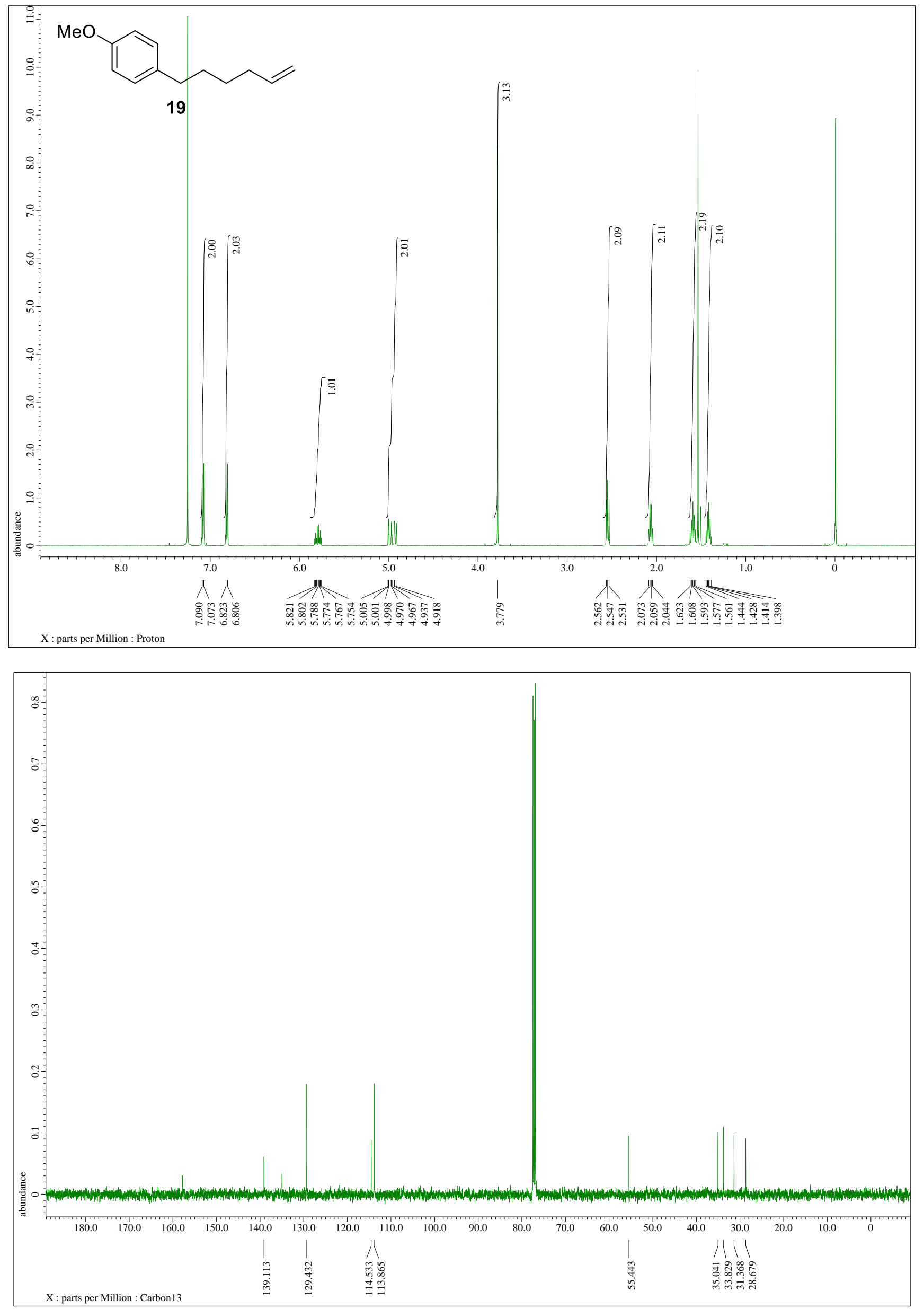

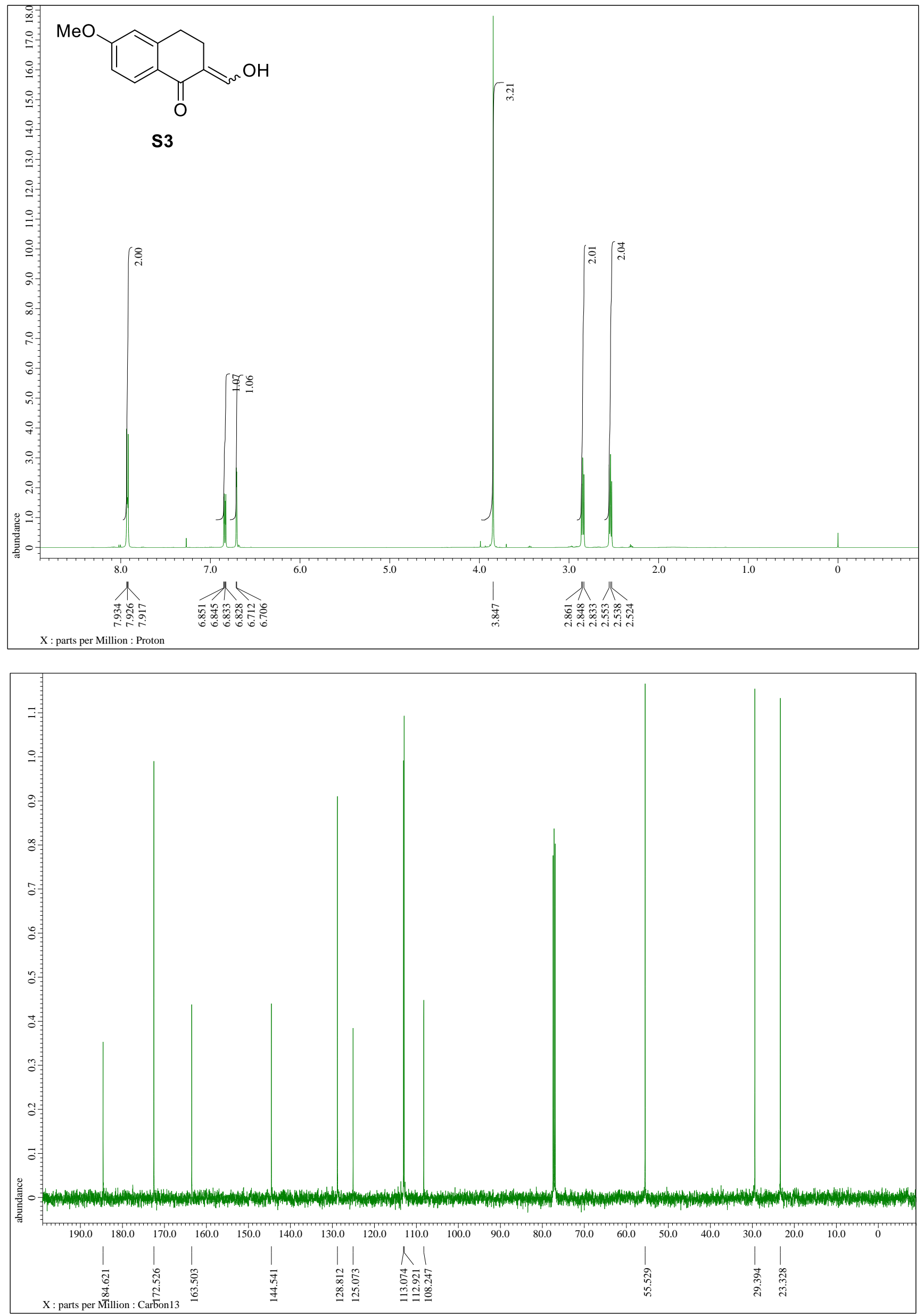

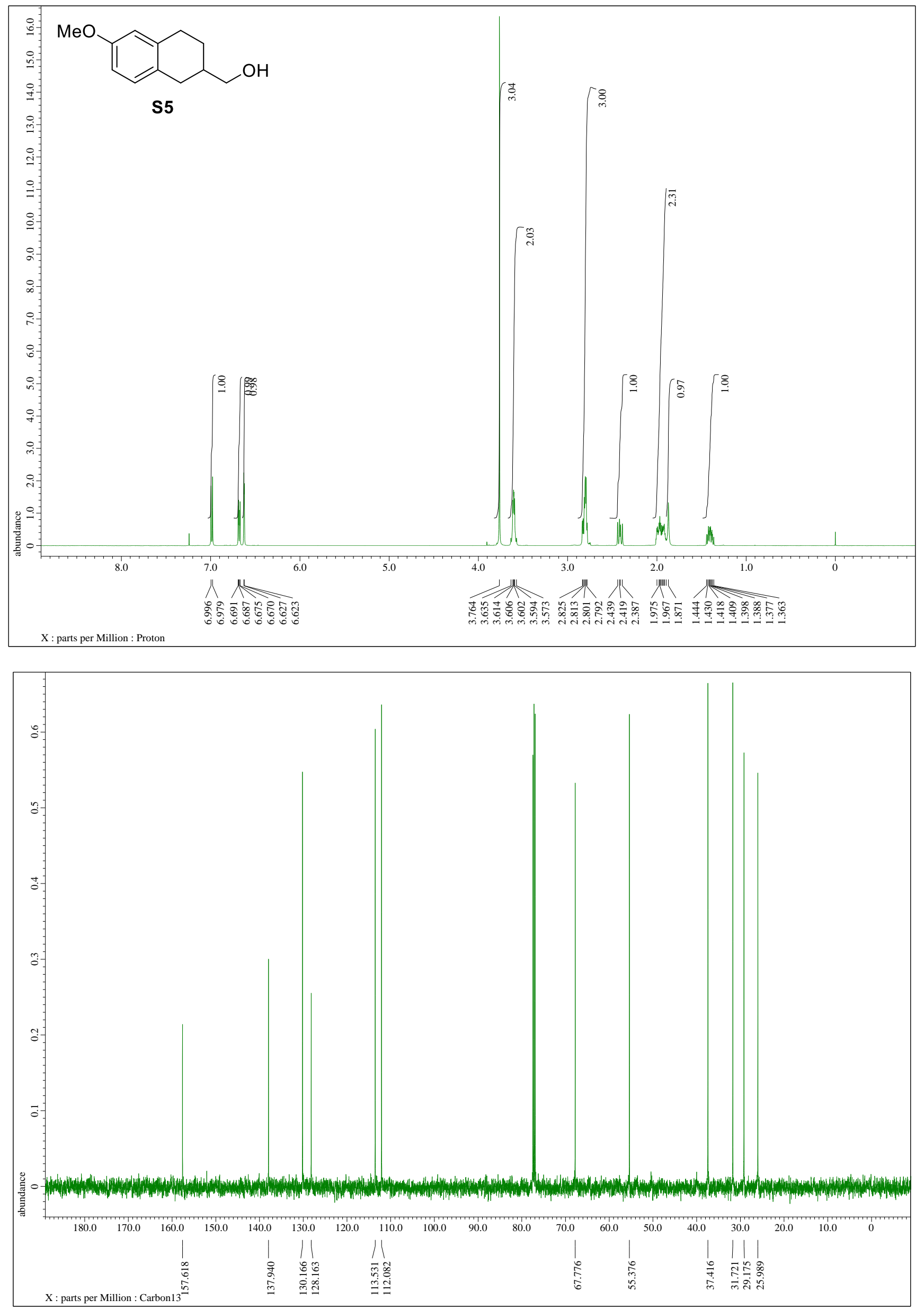

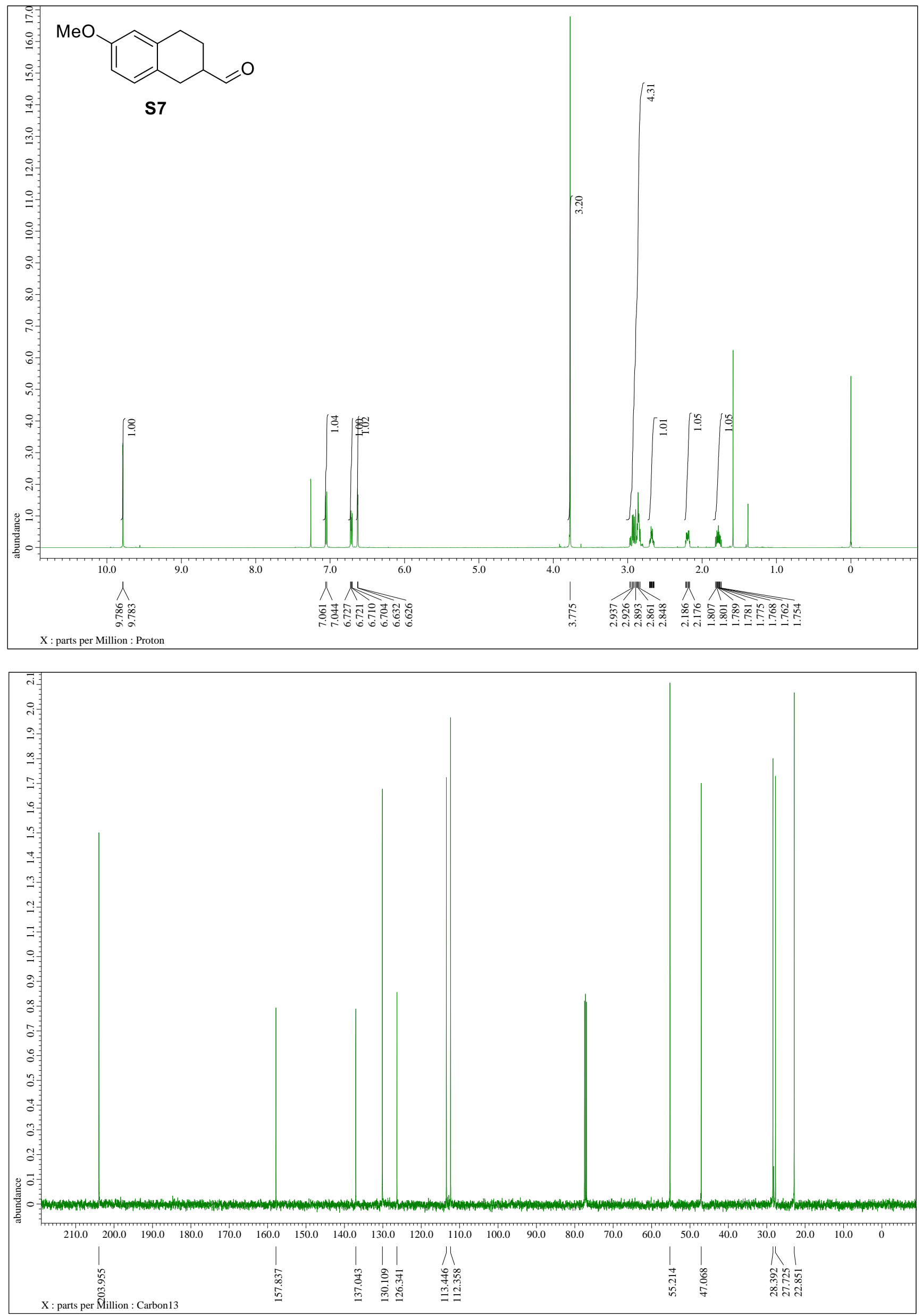

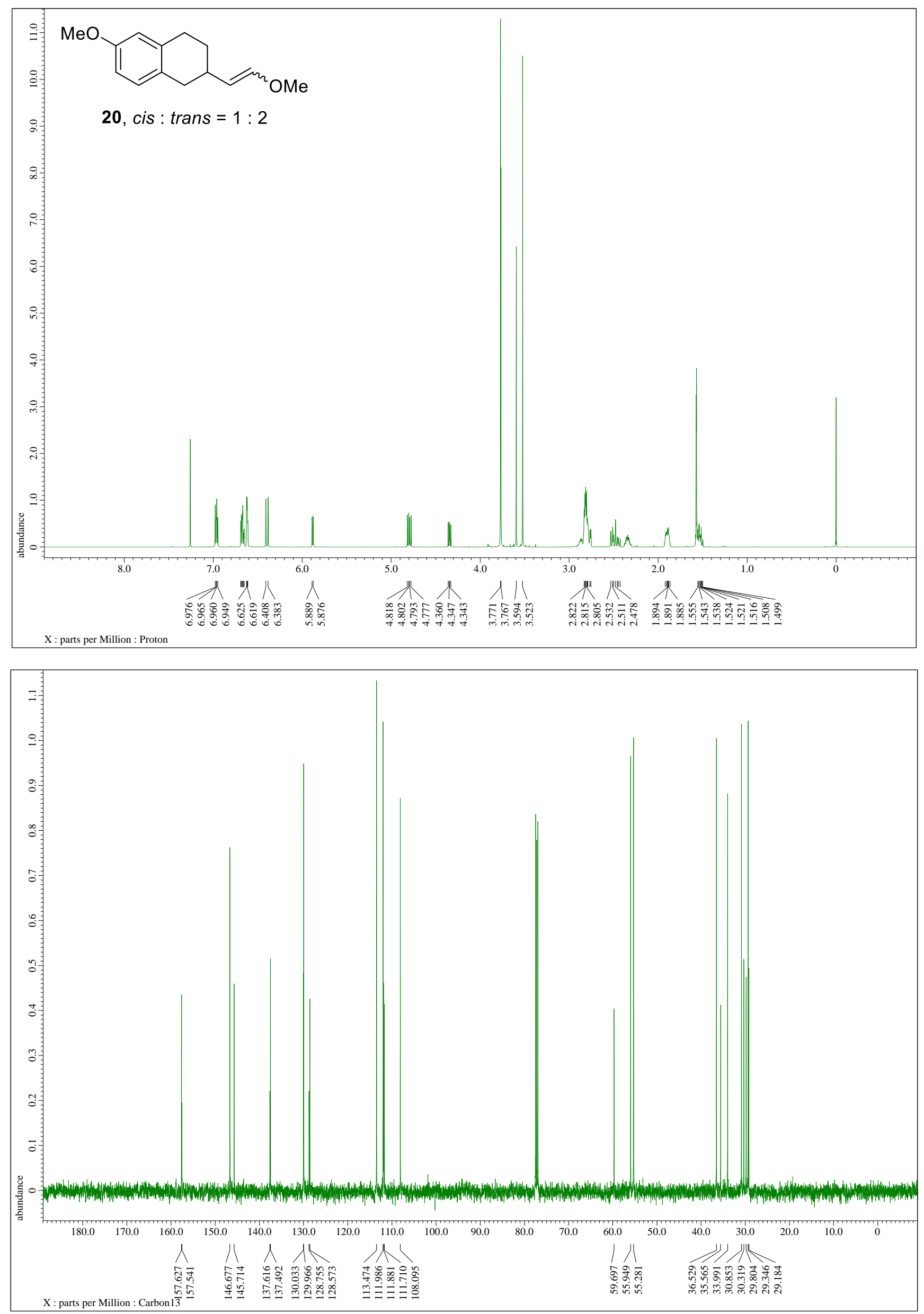

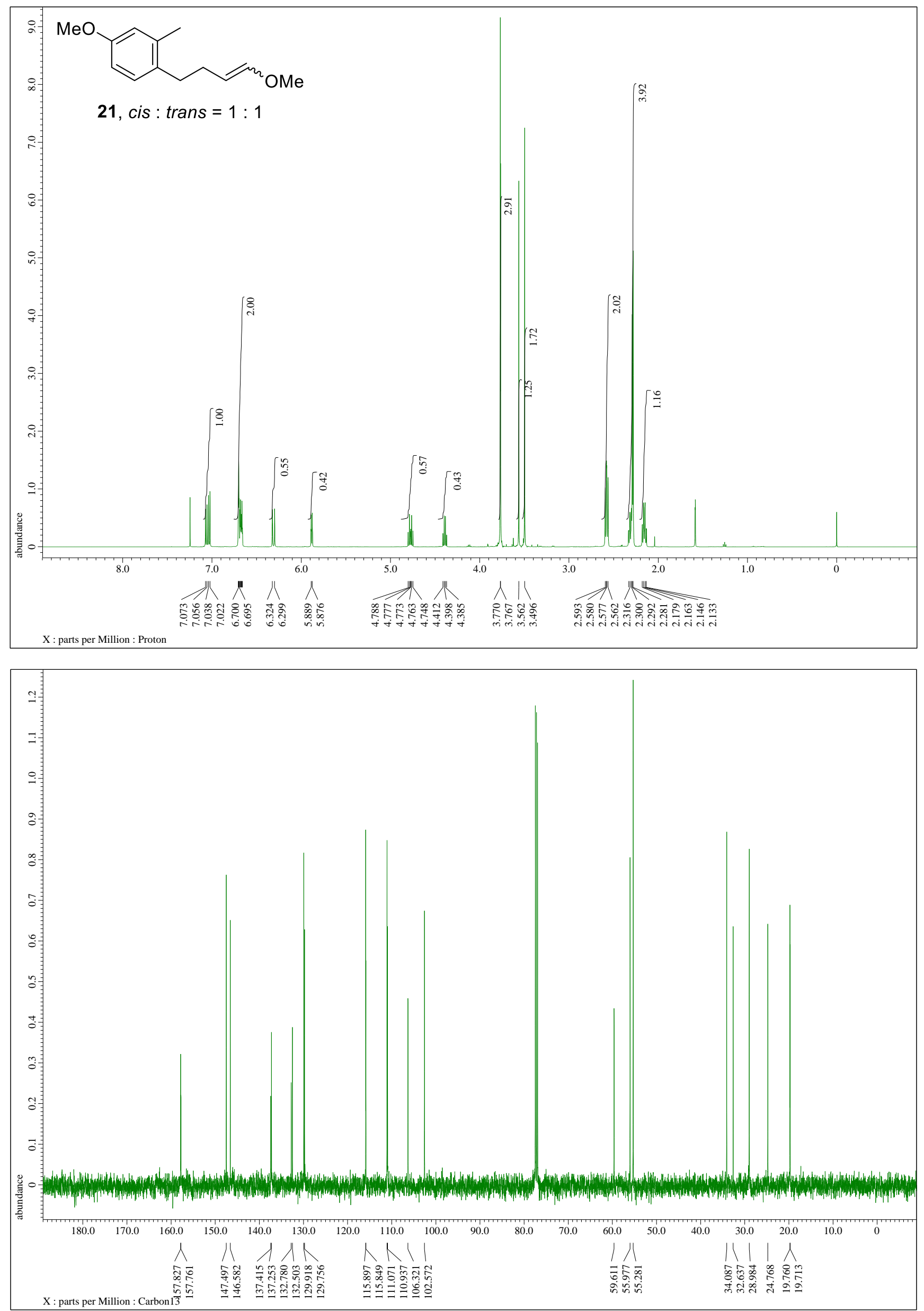

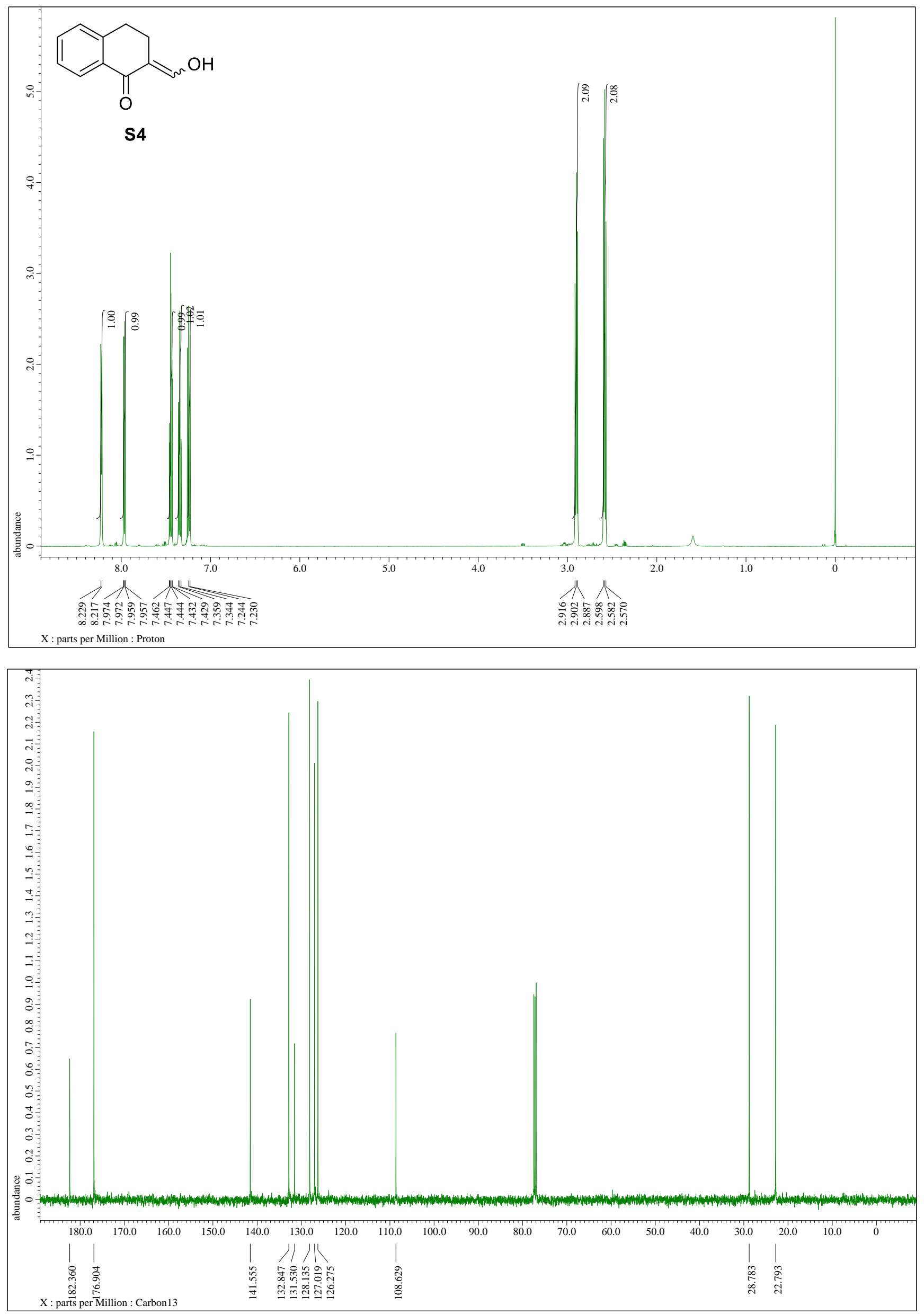

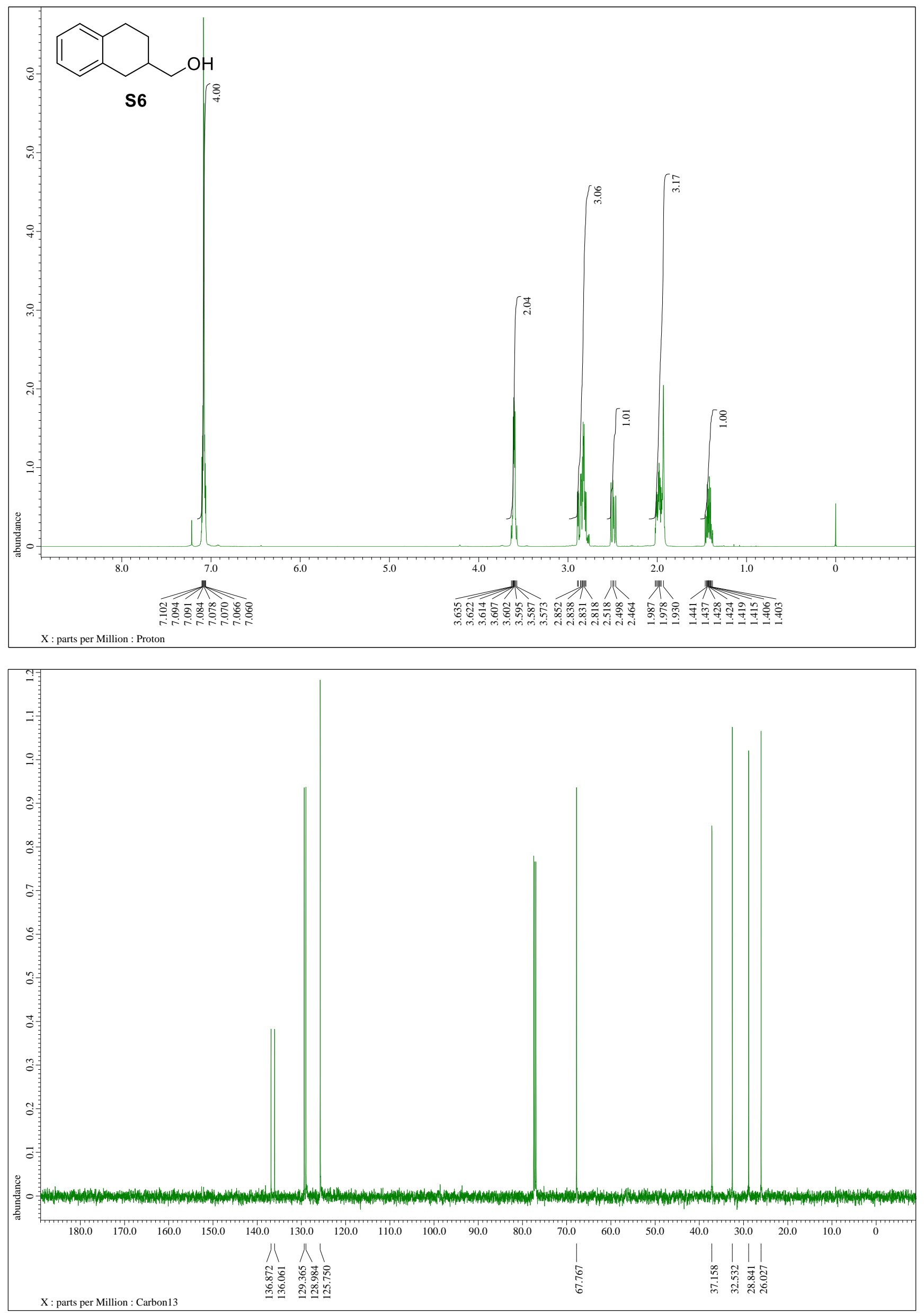

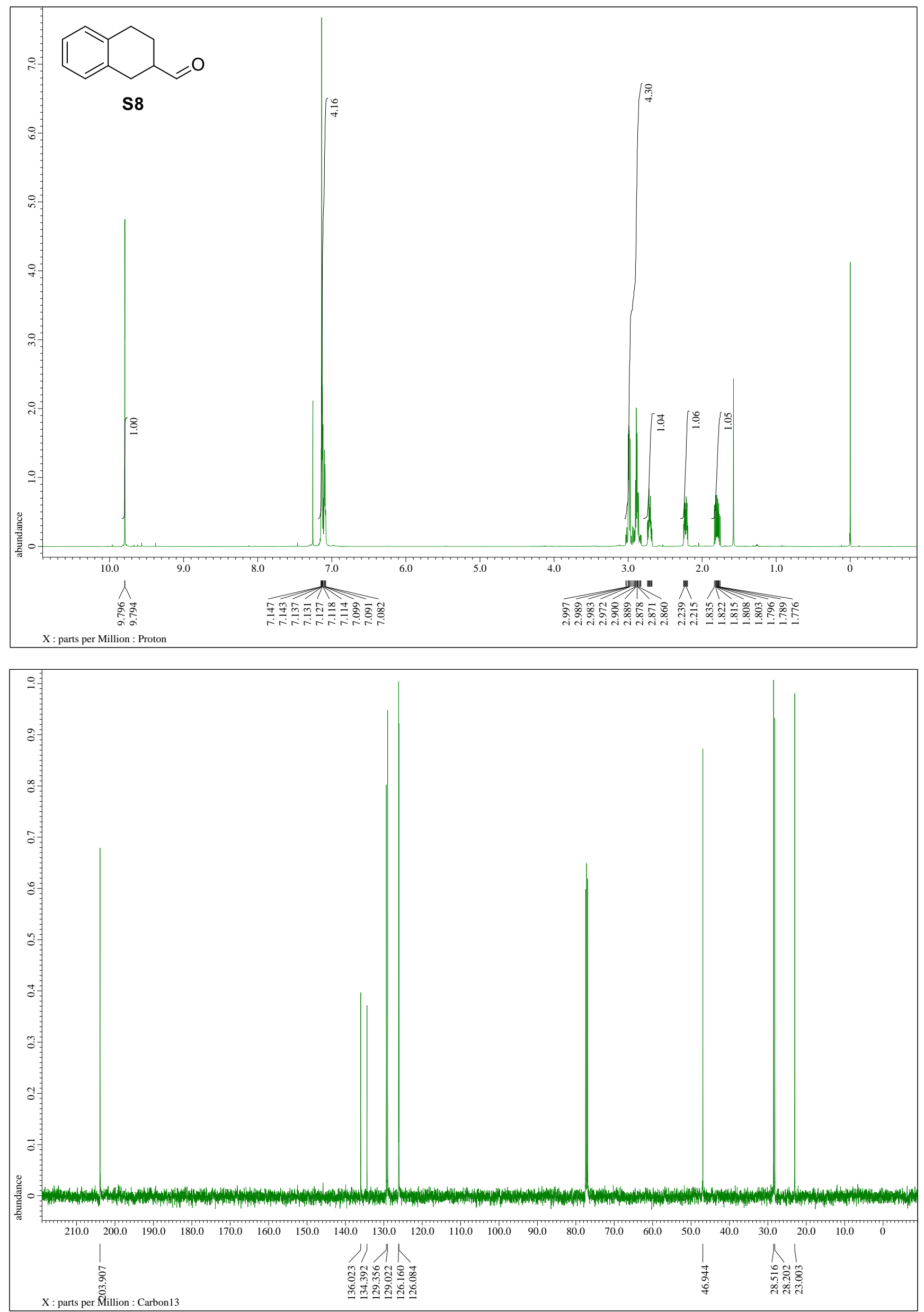

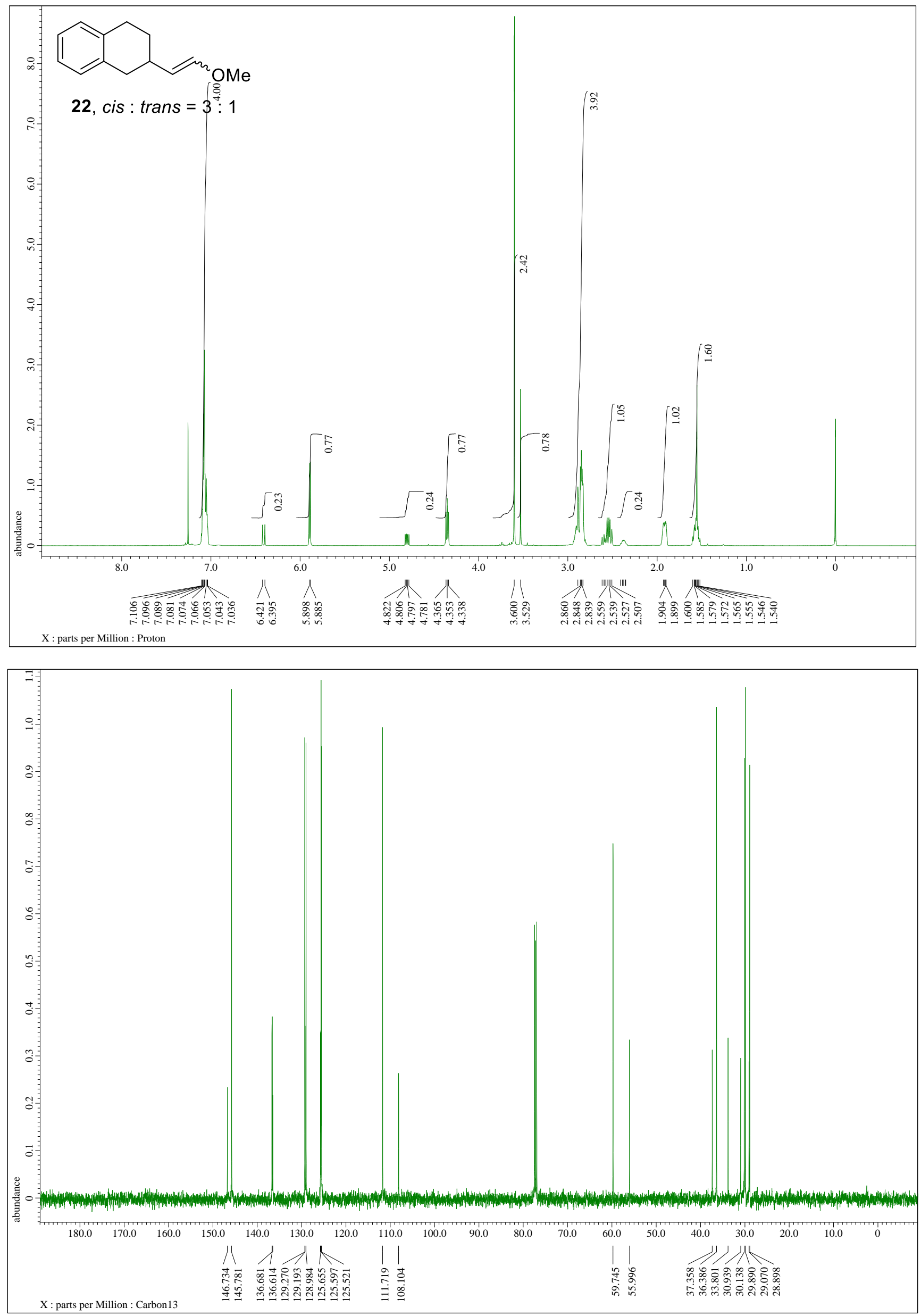

73 

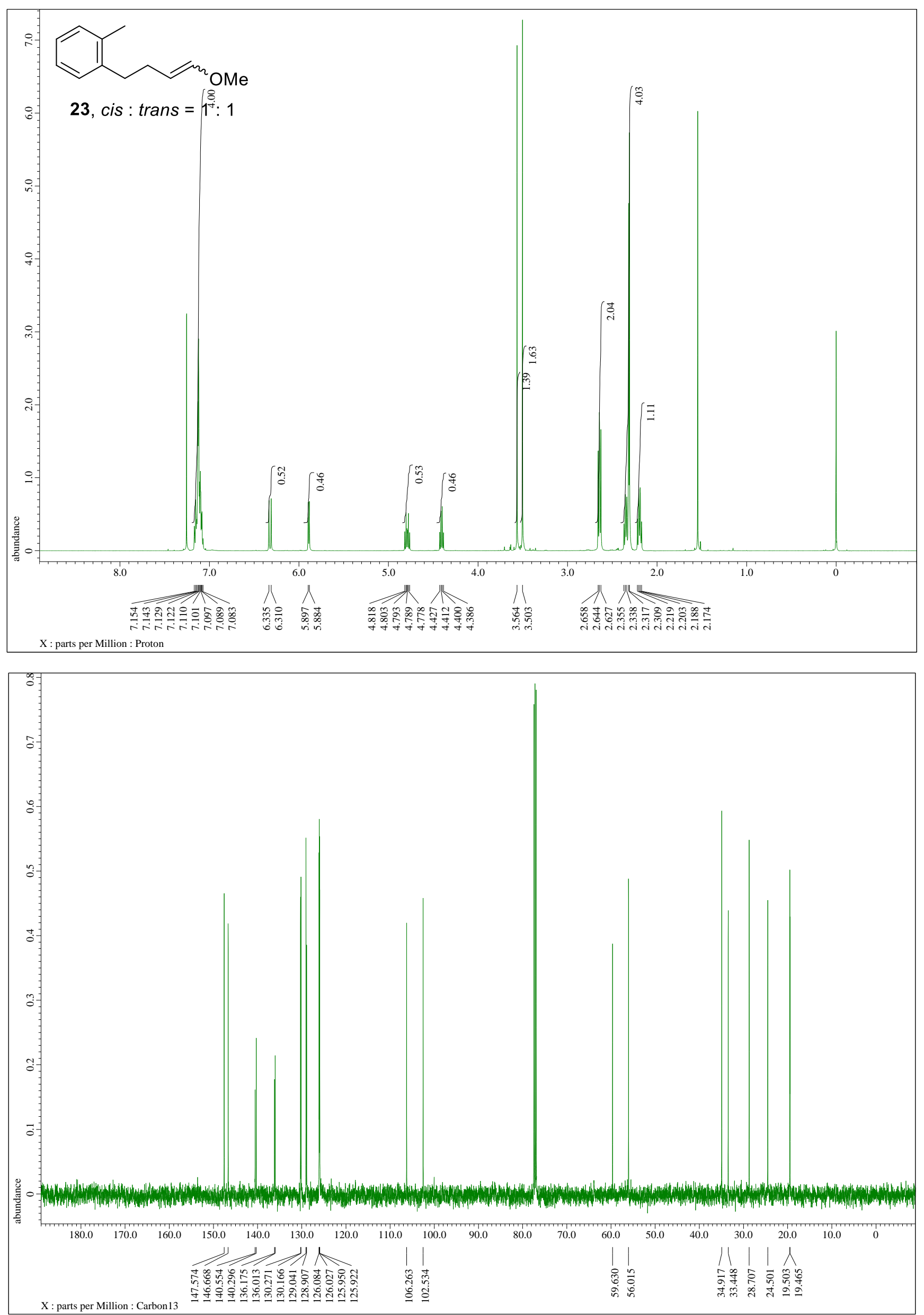

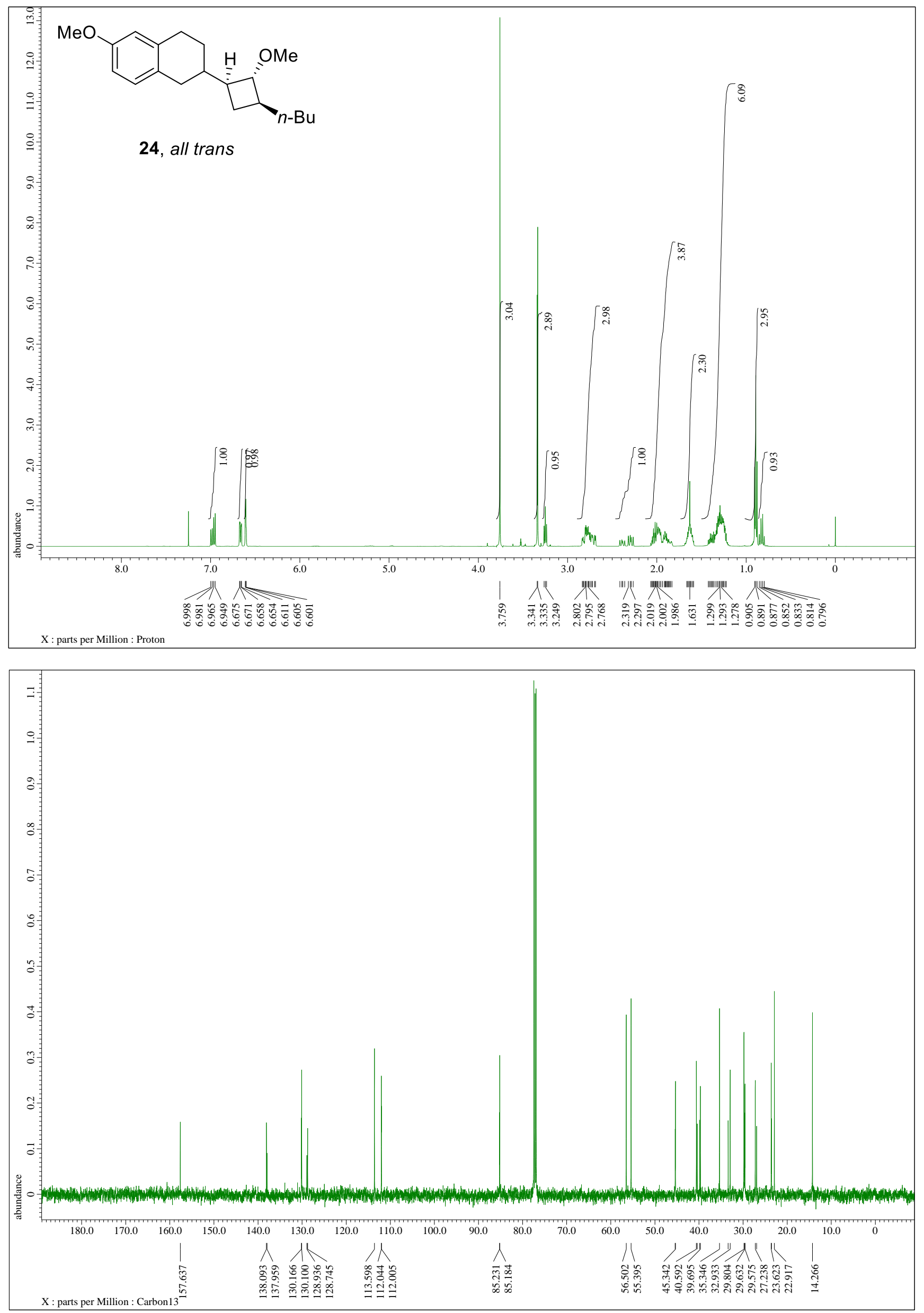

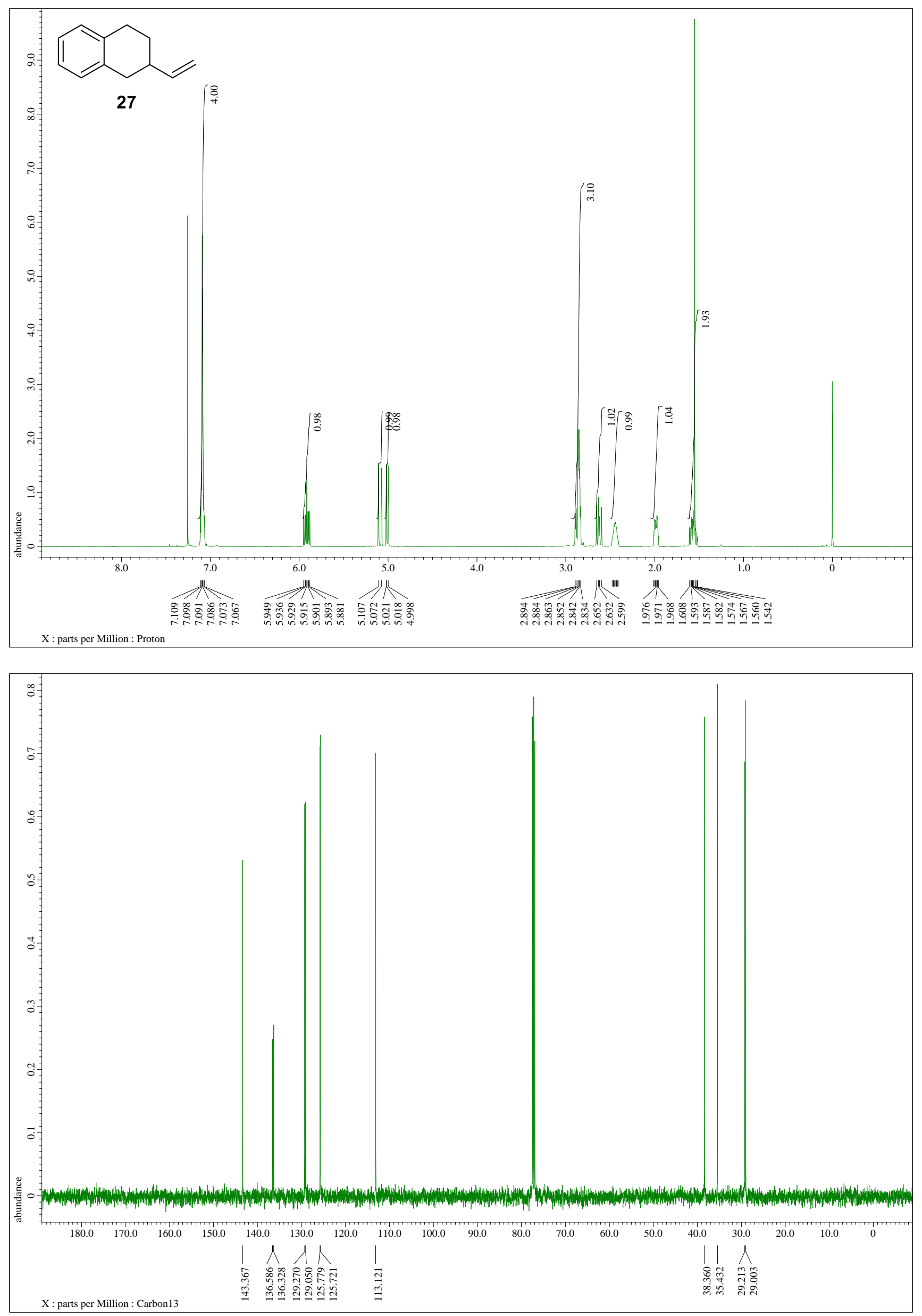

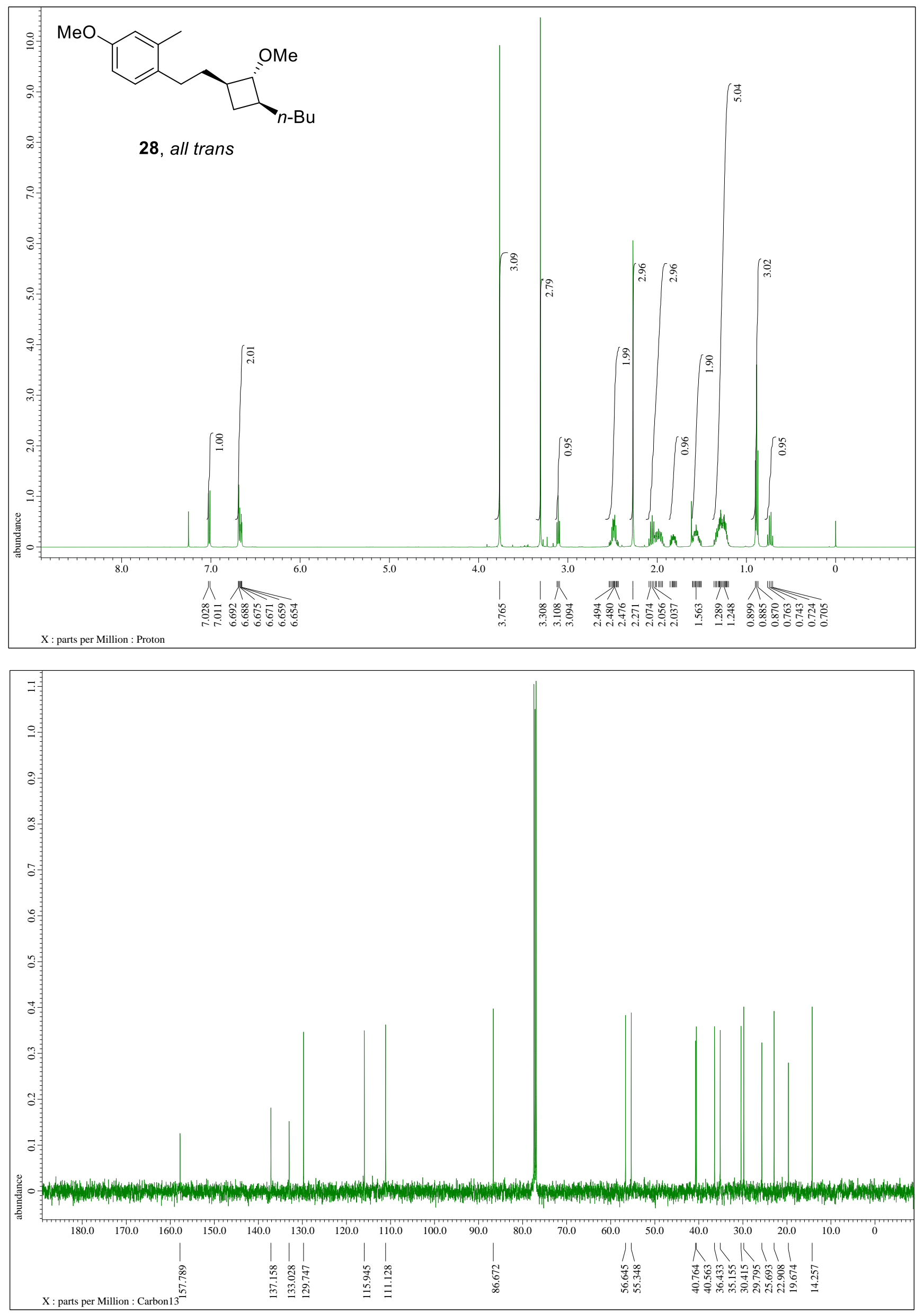

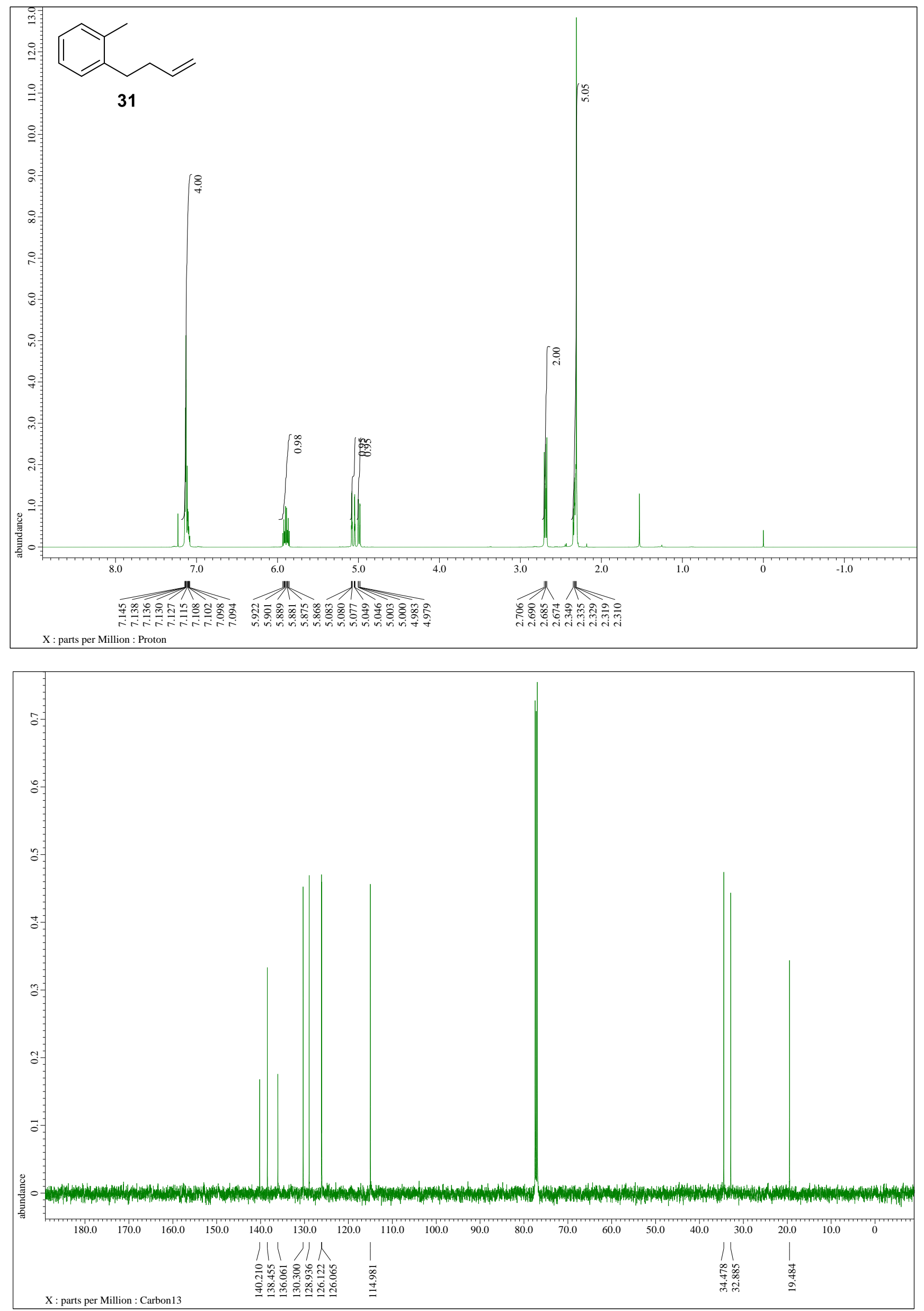